\title{
SUR LA FORMULE DE SIEGEL DANS LA THÉORIE DES GROUPES CLASSIQUES \\ PAR
}

\author{
ANDRE WEIL
}

The Institute for Advanced Study, Princeton, N.J., U.S.A.

A la fin d'un précédent mémoire ([14], $\mathrm{n}^{\circ} 52$ ), j'ai énoncé une formule qui (sous la forme un peu plus générale qui lui sera donnée ici) contient la plupart des résultats obtenus par Siegel dans ses travaux sur les formes quadratiques. En substance, il s'agit d'une identité dont le second membre est une " série d'Eisenstein 》, tandis que le premier s'obtient en écrivant une série (qui généralise les séries thêta) dépendant de certains paramètres, et en intégrant par rapport à ceux-ci dans le domaine fondamental d'un certain groupe discret. Comme on le verra, on en déduit, entre autres applications, la détermination du " nombre de Tamagawa " de la plupart des groupes classiques, ainsi qu'une démonstration partielle, pour ces même groupes, du " principe de Hasse " et d'un « théorème d'approximation ".

On s'efforcera de traiter simultanément les divers types de groupes classiques en suivant l'idée de Siegel, qui consiste à se donner d'abord une algèbre semisimple à involution $\mathcal{A}$ et un module $X$ sur $\mathcal{A}$. On peut toujours d'ailleurs, comme on sait, se ramener, soit au cas (I) où $\mathcal{A}$ est simple, soit au cas (II) où $\mathcal{A}$ est somme de deux composantes simples échangées par l'involution donnée; le cas (I) lui-même se subdivise en plusieurs types (involutions de $1^{\mathrm{e}}$ et de $2^{\mathrm{e}}$ espèce, etc.). Il est vrai qu'en plusieurs circonstances je n'ai pu éviter de séparer ces cas, et le lecteur ne manquera pas d'être frappé, et peut-être rebuté, par les complications qui en résultent. A bien des égards, le cas (I) est le plus intéressant; il aurait été tentant de s'y limiter, si par malheur l'extension du corps de base (et en particulier la "localisation ", c'est-à-dire le passage de ce corps à l'une de ses complétions) ne faisait nécessairement passer de (I) à (II) lorsqu'on part d'une involution de $2^{\mathrm{e}}$ espèce, de sorte qu'on ne peut exclure le cas (II) sans exclure ces involutions. De même, on verra que les applications les plus intéressantes de nos résultats se présentent lorsqu'on prend pour $X$ un module simple sur $\mathcal{A}$; mais, lorsqu'on étend le corps de base, un $\mathcal{A}$-module simple cesse en général d'être tel, à moins que $\mathcal{A}$ ne soit une algèbre de matrices sur le corps 1-652922. Acta mathematica. 113. Imprimé le 25 février 1965. 
de base. Si on s'était une fois pour toutes borné à ces algèbres, et aux modules simples sur celles-ci, on aurait pu donner de la présente théorie un exposé bien plus court, de lecture plus agréable, et qui néanmoins en eût fait apparaître les traits essentiels. Aussi le lecteur peu familier avec ce genre de question fera-t-il bien, en première lecture, de garder seulement ce cas présent à l'esprit (en supposant même de plus que l'involution dans $\mathcal{A}$ soit donnée par une matrice symétrique, ce qui correspond à la théorie des formes quadratiques et du groupe orthogonal) et de négliger tout ce qui, dans ce cas, devient visiblement inutile ou trivial. Mais, s'il est une vérité que l'évolution des mathématiques contemporaines a mise en évidence, c'est qu'on ne simplifie pas une théorie compliquée, pour peu qu'elle ait quelque valeur, en la spécialisant mais en la généralisant; bien entendu, je ne fais pas allusion par là aux généralisations superficielles ou artificielles que suggère parfois la mode, mais à celles qui placent une théorie dans son cadre naturel en la débarrassant des circonstances adventices qui l'encombrent ou la défigurent. Or il n'est pas douteux que nous n'ayons affaire ici à un fragment de la future théorie arithmétique des groupes semisimples. Mais il peut être avantageux, en vue de progrès ultérieurs dans cette direction, de disposer d'un matériel de comparaison le plus étendu possible; c'est dans cet esprit que j'ai cherché à donner du moins à la méthode suivie ici (directement inspirée de celle de Siegel dans [12]) tout le degré de généralité qu'elle comporte, plutôt que d'en présenter un simple échantillon.

Nous aurons à nous appuyer sur les résultats obtenus dans ces dernières années par Borel et Harishchandra, par Borel, et par Godement, dans la théorie des groupes semisimples. La validité de ces résultats n'est provisoirement acquise que dans le cas de caractéristique 0; en conséquence, on se bornera exclusivement à ce cas à partir du Chapitre III. La plupart des résultats auxiliaires des Chapitres I et II sont valables, soit en toute caractéristique, soit du moins chaque fois que la caractéristique n'est pas 2 . Les principaux résultats et notations de [14] seront supposés connus du lecteur.

\section{Table des notations}

(Cf. aussi la table des notations de [14].)

Chapitre I

$\mathrm{n}^{0}$ 1: $F_{\Phi^{*}}^{*}$.

$\mathrm{n}^{\mathrm{0}}$ 2: $\boldsymbol{F}_{\Phi},(\mathrm{A}),(\mathrm{B}),\left(\mathrm{B}_{0}\right),\left(\mathrm{B}_{1}\right)$.

$\mathrm{n}^{\circ}$ 3: $|\omega|_{v}, X^{\circ}$.

$\mathrm{n}^{\circ}$ 4: $U_{v}^{\circ},|\lambda \omega|_{A}, \Delta_{G}$.

$\mathbf{n}^{0}$ 5: $\left(\omega / f_{*} \eta\right)_{y}$.

$n^{0}$ 8: (W).

$\mathrm{n}^{\circ}$ 9: $T, a_{\tau}, \Theta(T)$.

$\mathrm{n}^{\circ}$ 10: $T_{A}^{+}, \Theta^{+}$.
Chapitre II

$\mathrm{n}^{\mathrm{o}}$ 14: $\mathcal{A}, \iota, \tau, X, X^{*}$.

$\mathrm{n}^{\circ}$ 15: $\otimes_{\iota}, I(X), I(X) *, Q(X / \mathcal{A})$.

$\mathrm{n}^{\mathrm{o}}$ 16: $\mathcal{A}_{v}, A_{v}, A, \mathfrak{f}_{v}, \mathcal{B}, m_{v}, X, X_{v}, n_{v}$.

$\mathrm{n}^{\circ}$ 17: $B$.

$\mathrm{n}^{\circ}$ 18: (I), (II), f, $\xi \rightarrow \xi^{\prime}, h, \eta, \mathfrak{l}^{\prime}, \mathfrak{z}$, żo, $\tau_{m^{*}}$

$\mathrm{n}^{\circ} 19: h[x], \delta, \varepsilon$.

$\mathrm{n}^{\circ}$ 21: $\delta$.

$\mathrm{n}^{\circ}$ 22: $G, U(i)$. 


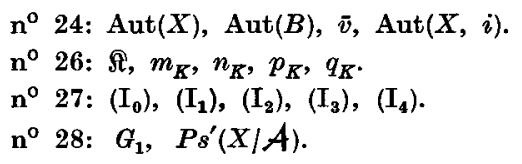

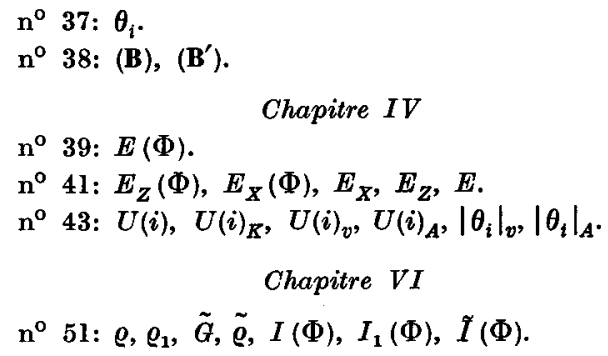

\section{Préliminaires analytiques}

1. Dans les $n^{\text {os }}$ qui vont suivre, on se donnera un groupe abélien localement compact $G$. Comme dans [14], on notera $G^{*}$ le dual de $G$, et $\left\langle g, g^{*}\right\rangle$ la valeur en $g$ du caractère de $G$ qui correspond à $g^{*} \in G^{*}$. On se donnera aussi une mesure de Haar $d g$ sur $G$, dont la duale sur $G^{*}$ sera notée $d g^{*}$. Rappelons que la transformation de Fourier définit un isomorphisme, d'une part entre $L^{2}(G)$ et $L^{2}\left(G^{*}\right)$, et d'autre part entre les espaces "de Schwartz-Bruhat " $S(G), S\left(G^{*}\right)$ respectivement attachés à $G$ et $G^{*}$ (cf. [14], $\mathrm{n}^{0} 11$ ). Par définition, une «distribution tempérée " sur $G$ est une forme linéaire continue sur $\boldsymbol{S}(G)$, c'est-à-dire que l'espace des distributions tempérées sur $G$ est le dual de $S(G)$. Il s'ensuit que la transformation de Fourier détermine un isomorphisme entre les espaces de distributions tempérées sur $G$ et sur $G^{*}$. En particulier, si $\varphi$ est une fonction bornée localement intégrable sur $G, \varphi(g) d g$ peut être considérée comme distribution tempérée, et admet donc une transformée de Fourier qui est une distribution tempérée sur $G^{*}$. Rappelons encore que les fonctions

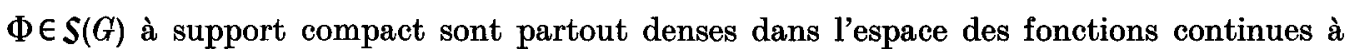
support compact sur $G$; on en conclut que, si une distribution tempérée $T$ sur $G$ est positive (c'est-à-dire si $T(\Phi) \geqslant 0$ chaque fois que $\Phi \geqslant 0$ ), elle définit une mesure (dite "tempérée ") sur $G$, et peut être identifiée avec celle-ci.

Dans ce qui va suivre, on se donnera aussi un espace localement compact $X$ muni d'une mesure $d x$, et une application continue $f$ de $X$ dans $G$; à partir du $\mathbf{n}^{\circ} 2, X$ sera un groupe abélien, et $d x$ une mesure de Haar sur $X$. A toute fonction $\Phi \in L^{1}(X)$, on conviendra d'associer la fonction $F_{\Phi}^{*}$ sur $G^{*}$, définie par la formule

$$
F_{\Phi}^{*}\left(g^{*}\right)=\int_{X} \Phi(x) \cdot\left\langle f(x), g^{*}\right\rangle d x
$$

dont on vérifie immédiatement qu'elle est continue et bornée. Avec ces notations, on a le lemme suivant : 
Lемме 1. Soit $\tau$ une fonction bornée localement intégrable sur $G^{*}$; soit $T$ la distribution tempérée sur $G$, transformée de Fourier de $\tau$. Pour tout voisinage compact $W$ de 0 dans $G$, soit $\varphi_{W}$ une fonction continue $\geqslant 0$ sur $G$, de support contenu dans $W$, telle que $\int \varphi_{W} d g=1$; et soit $t_{W}=\tilde{\varphi}_{W} * T * \varphi_{W}$. Alors les $t_{W}$ sont des fonctions continues et bornées sur $G$; et, chaque fois que $\Phi \in L^{1}(X)$ est telle que l'intégrale

$$
S(\Phi)=\int_{G^{*}} F_{\Phi}^{*}\left(-g^{*}\right) \tau\left(g^{*}\right) d g^{*}
$$

soit absolument convergente, on a aussi

$$
S(\Phi)=\lim _{W} \int_{X} \Phi(x) t_{W}(f(x)) d x
$$

la limite étant prise suivant l'ordonné filtrant des voisinages de 0 dans $G$. De plus, si la convergence absolue de (2) a lieu uniformément sur une partie bornée $B$ de $L^{1}(X)$, le second membre de (3) converge uniformément sur $B$.

Soit $\varphi_{W}^{*}$ la transformée de Fourier de $\varphi_{w} ;$ on a

$$
\left|\varphi_{W}^{*}\left(g^{*}\right)\right| \leqslant \int \varphi_{W} d g=1, \quad \int\left|\varphi_{W}^{*}\left(g^{*}\right)\right|^{2} d g^{*}=\int \varphi_{W}^{2} d g<+\infty .
$$

Comme il est bien connu, $t_{w}$ (qui est une " régularisée " de $T$ ) est la transformée de Fourier de $\left|\varphi_{W}^{*}\right|^{2} \tau$, c'est-à-dire qu'on a

$$
t_{W}(g)=\int\left|\varphi_{W}^{*}\left(g^{*}\right)\right|^{2} \tau\left(g^{*}\right) \cdot\left\langle g,-g^{*}\right\rangle d g^{*}
$$

c'est donc bien une fonction continue bornée sur $G$. En vertu du théorème de LebesgueFubini, on a, pour $\Phi \in L^{1}(X)$ :

$$
\int_{X} \Phi(x) t_{W}(f(x)) d x=\int_{G^{*}}\left(\int_{X} \Phi(x) \cdot\left\langle f(x),-g^{*}\right\rangle d x\right)\left|\varphi_{W}^{*}\left(g^{*}\right)\right|^{2} \tau\left(g^{*}\right) d g^{*},
$$

et par suite, si (2) est absolument convergente:

$$
S(\Phi)-\int_{X} \Phi(x) t_{W}(f(x)) d x=\int_{G^{*}}\left(1-\left|\varphi_{W}^{*}\left(g^{*}\right)\right|^{2}\right) F_{\Phi}^{*}\left(-g^{*}\right) \cdot \tau\left(g^{*}\right) d g^{*}
$$

Soit $K$ une partie compacte de $G^{*}$, et soit $\varepsilon>0$; il y a un voisinage $W_{0}$ de 0 dans $G$ tel que $\left|\left\langle g, g^{*}\right\rangle-1\right| \leqslant \varepsilon$ chaque fois que $g \in W_{0}$ et $g^{*} \in K$, et par suite

$$
\left|\varphi_{W}^{*}\left(g^{*}\right)-1\right|=\left|\int \varphi_{W}(g) \cdot\left(\left\langle g, g^{*}\right\rangle-1\right) d g\right| \leqslant \varepsilon
$$


chaque fois que $W \subset W_{0}$ et $g^{*} \in K$; autrement dit, on a $\lim _{w} \varphi_{W}^{*}=1$, uniformément sur toute partie compacte de $G^{*}$, la limite étant entendue comme il est dit dans l'énoncé du lemme 1. La première assertion du lemme résulte alors immédiatement de (4). Quant à la seconde, l'hypothèse signifie qu'à tout $\varepsilon>0$ on peut faire correspondre une partie compacte $K$ de $G^{*}$ telle que l'on ait

$$
\int_{G^{*}-K}\left|F_{\Phi}^{*}\left(-g^{*}\right) \tau\left(g^{*}\right)\right| d g^{*} \leqslant \varepsilon
$$

quel que soit $\Phi \in B$; cela étant, la conclusion s'ensuit aussi de (4).

2. Prenons maintenant pour $X$ un groupe abélien localement compact et pour $d x$ une mesure de Haar sur $X$. Nous aurons à nous servir de la remarque suivante, que pour plus de commodité nous énonçons sous forme de lemme :

LEMME 2. Soit $\left(S_{\alpha}\right)_{\alpha \in A}$ une famille de distributions tempérées sur $X$, et soit $\mathcal{I}$ un filtre sur $A$. Supposons que $\left(S_{\alpha}(\Phi)\right)_{\alpha \in A}$ converge suivant $\mathcal{I}$ pour tout $\Phi \in S(X)$, la convergence étant uniforme sur toute partie compacte de $S(X)$. Alors $S(\Phi)=\lim _{\mathfrak{F}} S_{\alpha}(\Phi)$ est une distribution tempérée.

Il est clair en effet que c'est une forme linéaire sur $S(X)$, continue sur toute partie compacte de $S(X)$. Comme $S(X)$ est limite inductive d'espaces métrisables $S\left(H, H^{\prime}\right)$ (cf. $\left.[14], \mathbf{n}^{\circ} 11\right)$, la conclusion s'ensuit immédiatement.

Lемме 3. Les hypothèses et notations étant celles du lemme 1 du $n^{\circ} 1$, supposons de plus que $X$ soit un groupe abélien localement compact, dx une mesure de Haar sur $X$, et que (2) soit absolument convergente pour tout $\Phi \in S(X)$ et le soit uniformément sur toute partie compacte de $S(X)$. Alors (2) définit une distribution tempérée $S$ sur $X$, de support contenu dans $f^{-1}$ (supp $T$ ); si $T$ est une mesure positive, il en est de même de $S$; et, si $T$ coïncide avec une mesure $\varrho(g) d g$ dans une partie ouverte $U$ de $G$, $\varrho$ étant une fonction continue dans $U, S$ coïncide avec la mesure $\varrho(f(x)) d x$ dans $f^{-1}(U)$.

D'après le lemme 1 , on peut aussi considérer $S$ comme définie au moyen de (3); les lemmes 1 et 2 montrent alors que $S$ est une distribution tempérée. L'assertion sur le support de $S$ résulte du fait que le support de $t_{W}$ est contenu dans $\operatorname{supp}(T)+W+(-W)$. Si $T$ est une mesure positive, on a $t_{W} \geqslant 0$ quel que soit $W$, done $S(\Phi) \geqslant 0$ pour $\Phi \geqslant 0$. Enfin, si $T$ coïncide avec $\varrho(g) d g$ dans $U$, on a $\lim _{W} t_{W}=\varrho$ uniformément dans toute partie compacte de $U$, donc $\lim _{W} t_{W} \circ f=\varrho \circ f$ uniformément dans toute partie compacte de $f^{-1}(U)$, d'où la dernière assertion du lemme, en vertu de (3). 
Proposition 1. Soient $X$ et $G$ des groupes abéliens localement compacts; soit $f$ une application continue de $X$ dans $G$, satisfaisant à la condition suivante :

(A) quelle que soit $\Phi \in S(X)$, la fonction $F_{\Phi}^{*}$ définie par (1) est intégrable sur $G^{*}$; et l'intégrale $\int\left|F_{\Phi}^{*}\right| d g^{*}$ converge uniformément sur toute partie compacte de $S(X)$.

Alors on peut, d'une manière et d'une seule, faire correspondre à tout $g \in G$ une mesure positive $\mu_{g}$ sur $X$, de support contenu dans $f^{-1}(\{g\})$, de telle sorte que, pour toute fonction $\Phi$ continue à support compact sur $X$, la fonction $F_{\Phi}$ sur $G$ définie par $F_{\Phi}(g)=\int \Phi d \mu_{g}$ soit continue et satisfasse à $\int F_{\Phi} d g=\int \Phi d x$. De plus, les $\mu_{g}$ sont des mesures tempérées; et, pour tout $\Phi \in \mathcal{S}(X), F_{\Phi}$ est continue, appartient $\grave{a} L^{1}(G)$, satisfait à $\int F_{\Phi} d g=\int \Phi d x$ et est la transformée de Fourier de $F_{\Phi}^{*}$.

Si (A) est satisfaite, on peut, quel que soit $g$, appliquer le lemme 3 à $f$ et à la « distribution de Dirac " $T=\delta_{g}$, c'est-à-dire à la masse 1 concentrée en $g$. Il existe donc une mesure tempérée positive $\mu_{g}$, de support contenu dans $f^{-1}(\{g\})$, telle que, pour tout $\Phi \in S(X)$, $F_{\Phi}(g)=\int \Phi d \mu_{g}$ soit la valeur en $g$ de la transformée de Fourier de $F_{\Phi}^{*}$; puisque $F_{\Phi}^{*} \in L^{1}\left(G^{*}\right)$ pour $\Phi \in S(X), F_{\Phi}$ est alors continue et bornée. Pour $\Phi \in S(X), \Phi \geqslant 0$, on a $F_{\Phi} \geqslant 0$; donc $F_{\Phi}^{*}$ est alors une fonction continue de type positif, et par suite est transformée de Fourier d'une mesure positive bornée; celle-ci ne peut être autre que $F_{\Phi}(g) d g$, ce qui montre que $F_{\Phi}$ appartient alors à $L^{1}(G)$. Mais (par exemple en vertu du lemme 5 de [14], $\mathrm{n}^{\circ} 41$ ) toute fonction $\Phi \in S(X)$ s'écrit, si elle est réelle, comme différence de deux fonctions positives de $S(X)$, donc en tout cas comme combinaison linéaire finie de telles fonctions. On a donc $F_{\Phi} \in L^{1}(G)$ quelle que soit $\Phi \in S(X)$. Il s'ensuit que $F_{\Phi}^{*}$ est alors la transformée de Fourier de $F_{\Phi}$; en particulier, on a $F_{\Phi}^{*}(0)=\int F_{\Phi} d g$; comme on a $F_{\Phi}^{*}(0)=\int \Phi d x$ par définition de $F_{\Phi}^{*}$, cela donne bien, pour $\Phi \in S(X)$, l'égalité annoncée. Soit alors $\Phi$ une fonction continue sur $X$, dont le support $C$ soit compact; soient $U$ un voisinage compact de 0 dans $X$, et $C^{\prime}=C+U$. Il est facile de voir qu'il existe une fonction $\Psi \in S(X)$ qui soit $\geqslant 0$ sur $X$ et $\geqslant 1$ sur $C^{\prime}$, et, quel que soit $\varepsilon>0$, une fonction $\Phi^{\prime} \in S(X)$, de support contenu dans $C^{\prime}$, telle que $\left|\Phi^{\prime}-\Phi\right| \leqslant \varepsilon$. Alors les supports de $F_{\Phi}, F_{\Phi}$ sont contenus dans $f\left(C^{\prime}\right)$, et on a $\left|F_{\Phi^{\prime}}-F_{\Phi}\right| \leqslant \varepsilon F_{\Psi}$. On en conclut aussitôt que $F_{\Phi}$ est continue et qu'on a $\int F_{\Phi} d g=\int \Phi d x$. Il reste à démontrer l'unicité des $\mu_{g}$. Soit en effet $\left(\mu_{g}^{\prime}\right)$ une famille de mesures ayant les propriétés énoncées dans la proposition 1 , et soit $\mu_{g}^{\prime \prime}=\mu_{g}^{\prime}-\mu_{g}$. On aura donc, pour toute fonction continue $\Phi$ à support compact :

$$
\int\left(\int \Phi(x) d \mu_{g}^{\prime \prime}(x)\right) d g=0 .
$$

En remplaçant $\Phi(x)$ par $\Phi(x) \varphi(f(x))$, où $\varphi$ est une fonction continue sur $G$, on obtient 


$$
\int\left(\int \Phi(x) d \mu_{g}^{\prime \prime}(x)\right) \varphi(g) d g=0
$$

Cette relation doit être satisfaite quelle que soit $\varphi$; comme d'autre part les hypothèses faites entraînent que $\int \Phi d \mu_{g}^{\prime \prime}$ est une fonction continue de $g \in G$, il s'ensuit bien que celle-ci doit s'annuler quelle que soit $\Phi$, donc que $\mu_{g}^{\prime \prime}=0$.

Proposition 2. Soient $G$ un groupe abélien localement compact, $\Gamma$ un sous-groupe discret de $G$ tel que $G / \Gamma$ soit compact, et $\Gamma_{*}$ le sous-groupe discret de $G^{*} q u i$ correspond par dualité à $\Gamma$. Soient $X$ un groupe abélien localement compact et $f$ une application continue de $X$ dans $G$ satisfaisant à la condition suivante :

(B) quels que soient $\Phi \in S(X)$ et $g^{*} \in G^{*}$, la série

$$
\sum_{\gamma^{*} \in \Gamma_{*}}\left|F_{\Phi}^{*}\left(g^{*}+\gamma^{*}\right)\right|
$$

est convergente, et elle l'est uniformément sur toute partie compacte de $S(X) \times G^{*}$.

Alors $f$ satisfait à la condition $(\mathrm{A})$ de la proposition 1 . De plus, si $\boldsymbol{F}_{\Phi}$ désigne de nouveau la transformée de Fourier de la fonction $F_{\Phi}^{*}$ définie par $(1)$, on a, pour toute fonction $\Phi \in S(X)$ :

$$
\sum_{\gamma \in \Gamma} F_{\Phi}(\gamma)=\sum_{\gamma^{*} \in \Gamma^{*}} F_{\Phi}^{*}\left(\gamma^{*}\right)
$$

les séries des deux membres étant absolument convergentes.

La première assertion signifie que, si $\varepsilon>0$, et si $C$ est une partie compacte de $S(X)$, il existe une partie compacte $K$ de $G^{*}$ telle que l'intégrale de $\left|F_{\Phi}^{*}\right|$ sur $G^{*}-K$ soit $\leqslant \varepsilon$ quelle que soit $\Phi \in C$. Or, comme $\Gamma$ est discret, $G^{*} / \Gamma_{*}$ est compact; il existe done une partie compacte $C^{*}$ de $G^{*}$ telle que $G^{*}=C^{*}+\Gamma_{*}$. Alors, d'après (B), il existe, quel que soit $\delta>0$, une partie finie $F$ de $\Gamma_{*}$ telle que l'on ait

$$
\sum_{\gamma^{*} \in \Gamma_{*}-F}\left|F_{\Phi}^{*}\left(g^{*}+\gamma^{*}\right)\right| \leqslant \delta
$$

chaque fois que $\Phi \in C$ et $g^{*} \in C^{*}$. On satisfera alors à la condition imposée en prenant $\delta \leqslant m\left(C^{*}\right)^{-1} \varepsilon$, où $m\left(C^{*}\right)$ est la mesure de $C^{*}$ pour $d x$, puis $K=C^{*}+F$.

On peut donc appliquer à $f$ la proposition 1; par suite, il existe des mesures $\mu_{g}$ avec les propriétés énoncées dans celle-ci. D'autre part, soit $\pi$ l'homomorphisme canonique de $G$ sur $G / \Gamma$, et soit $f=\pi \circ f$. Comme $\Gamma_{*}$ est le dual de $G / \Gamma$, (B) entraîne que l'application $f$ de $X$ dans $G / \Gamma$ satisfait à la condition (A) de la proposition 1 . Il existe donc pour tout $g$ une mesure tempérée positive $\nu_{\pi(g)}$, de support contenu dans la réunion des $f^{-1}(\{g+\gamma\})$ pour $\gamma \in \Gamma$, telle que l'on ait, pour tout $\Phi \in S(X)$ : 


$$
\int \Phi d \nu_{\pi(g)}=\sum_{\gamma^{*} \in \Gamma^{*}} F_{\Phi}^{*}\left(\gamma^{*}\right) \cdot\left\langle g,-\gamma^{*}\right\rangle,
$$

le premier membre étant une fonction continue. En particulier, prenons pour $\Phi$ une fonction à support compact $C$; comme $F_{\Phi}(g)=\int \Phi d \mu_{g}$ d'après la proposition 1 , le support de $F_{\Phi}$ est contenu dans $f(C)$. Alors la fonction $\bar{F}$ définie par

$$
\bar{F}(g)=\sum_{\gamma \in \Gamma} F_{\Phi}(g+\gamma)=\int \Phi \cdot\left(\sum_{\gamma \in \Gamma} d \mu_{g+\gamma}\right)
$$

est continue et détermine, par passage au quotient, une fonction continue sur $G / \Gamma$, dont les coefficients de Fourier sont donnés par

$$
c\left(\gamma^{*}\right)=\int_{G / \Gamma} \bar{F}(g) \cdot\left\langle g, \gamma^{*}\right\rangle d \dot{g}=\int_{G} F_{\Phi}(g) \cdot\left\langle g, \gamma^{*}\right\rangle d g=F_{\Phi}^{*}\left(\gamma^{*}\right)
$$

dans le second membre, on a posé $\dot{g}=\pi(g)$. Comme ces coefficients de Fourier sont les mêmes que ceux de $\int \Phi d v_{\pi(g)}$ tels qu'ils apparaissent dans (6), et que cette dernière fonction est continue, on en conclut qu'on a, pour toute fonction $\Phi \in S(X)$ à support compact :

$$
\int \Phi d v_{\pi(g)}=\int \Phi \cdot\left(\sum_{\gamma} d \mu_{g+\gamma}\right)
$$

Cela implique $\nu_{\pi(g)}=\sum \mu_{g+\gamma}$. Comme $\boldsymbol{v}_{\boldsymbol{\pi}(g)}$ est une mesure tempérée, il s'ensuit que la série $\sum F_{\Phi}(g+\gamma)$ est absolument convergente pour toute fonction positive $\Phi \in S(X)$, et par suite aussi pour toute $\Phi \in S(X)$. En écrivant (6) pour $g=0$, et tenant compte de ce qu'on vient de démontrer, on obtient la seconde partie de la proposition.

On peut exprimer la seconde partie de la proposition 2 en disant que la formule de Poisson est valable pour les fonctions $F_{\Phi}, F_{\Phi}^{*}$; cela serait trivial si ces fonctions appartenaient respectivement à $\boldsymbol{S}(G)$ et $\boldsymbol{S}\left(G^{*}\right)$, mais des exemples simples montrent qu'en général ce n'est pas le cas.

D'autre part, convenons, pour $\Phi \in S(X)$, de noter $\Phi_{g^{*}}$ la fonction définie par $\Phi_{g^{*}}(x)=$ $\Phi(x)\left\langle f(x), g^{*}\right\rangle$. Nous aurons à appliquer la proposition 2 à des fonctions $f$ satisfaisant aux conditions suivantes :

$\left(\mathrm{B}_{0}\right)\left(\Phi, g^{*}\right) \rightarrow \Phi_{g^{*}}$ est une application continue de $S(X) \times G^{*}$ dans $S(X)$;

$\left(\mathrm{B}_{1}\right)$ la série $\sum_{\gamma^{*} \in \Gamma^{*}}\left|F_{\Phi}^{*}\left(\gamma^{*}\right)\right|$ est uniformément convergente sur toute partie compacte de $\mathbf{S}(\boldsymbol{X})$.

Si on remplace $\Phi \operatorname{par} \Phi_{g_{0}^{*}}, F_{\Phi}^{*}\left(g^{*}\right)$ se change évidemment en $F_{\Phi}^{*}\left(g^{*}+g_{0}^{*}\right)$; on en conclut que $\left(B_{0}\right)$ et $\left(B_{1}\right)$ entraînent $(B)$, donc que la proposition 2 est applicable à toute fonction qui satisfait ces deux conditions. 
3. Nous allons maintenant compléter ce qui a été dit dans [14] concernant les espaces «adéliques 》 (cf. [14], $\mathrm{n}^{\text {os }}$ 29-30), et tout d'abord fixer le choix des mesures dans ces espaces.

On s'appuyera sur l'observation générale suivante. Soit $G$ un groupe abélien localement compact, et soit $\chi(x, y)$ un bicaractère non dégénéré de $G \times G$, c'est-à-dire une fonction de la forme $\langle x, y \beta\rangle$, où $\beta$ est un isomorphisme de $G$ sur $G^{*}$. On peut alors identifier $G$ avec son dual au moyen de $\chi$ en posant $\langle x, y\rangle=\chi(x, y)$, ce qui revient à les identifier au moyen de l'isomorphisme $\beta$; c'est ce que nous avons fait, en substance, pour les corps locaux au $n^{\circ} 24$ de [14], et pour les anneaux d'adèles au $n^{\circ} 29$ de [14]. Cela posé, on voit immédiatement qu'il existe un choix et un seul de la mesure de Haar pour $G$ pour laquelle celle-ci soit sa propre duale dans cette identification; on dira que cette mesure est autoduale par rapport à $\chi$.

Conservant alors nos notations habituelles (v. [14], $\mathrm{n}^{\circ} 29$ ), nous conviendrons une fois pour toutes de choisir sur la complétion $k_{v}$ du corps de base $k$ la mesure de Haar autoduale par rapport au bicaractère $\chi_{v}(x y)$, et de noter celle-ci $|d x|_{v}$. Alors, pour presque tout $v$, la mesure de l'anneau $\mathfrak{o}_{v}$ des entiers de $k_{v}$ a la valeur 1 .

Soit maintenant $U$ une variété analytique de dimension $n$ sur $k_{v}$; le choix de la mesure $|d x|_{v}$ sur $k_{v}$ permet, comme on sait (cf. p. ex. [13], Chap. II), de déduire de toute forme différentielle holomorphe $\omega$ de degré $n$ sur $U$ une mesure positive sur $U$, notée $|\omega|_{v}$. Rappelons que, si $U$ est une partie ouverte de $\left(k_{v}\right)^{n}$ et que $\omega$ y soit donnée par $\omega=f(x) d x_{1} \wedge d x_{2} \wedge \ldots \wedge d x_{n},|\omega|_{v}$ sera définie par

$$
|\omega|_{v}=|f(x)|_{v} \prod_{i=1}^{n}\left|d x_{i}\right|_{v}
$$

où $|a|_{v}$ désigne naturellement, pour tout $a \in k_{v}$, le module de l'automorphisme $x \rightarrow a x$ de $k_{v}$ si $a \neq 0$, et 0 si $a=0$. La définition de $|\omega|_{v}$ dans le cas général se déduit immédiatement de là, par transport de structure au moyen de cartes locales.

En particulier, soit $X_{k}$ un espace vectoriel de dimension $n$ sur $k$; soient $X_{k}^{*}$ son dual, $X^{\circ}$ une base de $X_{k}$ sur $k$, et $\left(X^{*}\right)^{\circ}$ la base de $X_{k}^{*}$ duale de $X^{\circ}$. Notons $X$, comme d'habitude, l'extension de $X_{k}$ au domaine universel; soient $x_{1}, \ldots, x_{n}$ les coordonnées dans $X$ par rapport à la base $X^{\circ}$, et soient de même $x_{1}^{*}, \ldots, x_{n}^{*}$ les coordonnées dans $X^{*}$ par rapport à $\left(X^{*}\right)^{\circ}$. Posons $d x=d x_{1} \wedge \ldots \wedge d x_{n}, d x^{*}=d x_{1}^{*} \wedge \ldots \wedge d x_{n}^{*}$; alors, si on tient compte des identifications faites aux $n^{\text {os }} 24$ et 29 de [14], les mesures $|d x|_{v}$ dans $X_{v}$ et $\left|d x^{*}\right|_{v}$ dans $X_{v}^{*}$ sont duales l'une de l'autre. C'est toujours ainsi qu'on choisira les mesures dans les espaces de la forme $X_{v}, X_{v}^{*}$ dans ce qui suit, étant entendu que ce choix est relatif au choix du caractère $\chi$ de $A_{k}$, supposé fait une fois pour toutes, et à celui des bases duales $X^{\circ},\left(X^{*}\right)^{\circ}$.

Dans l'anneau $A_{k}$ des adèles de $k$, la " mesure de Tamagawa " (celle pour laquelle $A_{k} / k$ est de mesure 1) est autoduale par rapport à $\chi(x y)$, puisque $k$ est associé à lui-même par 
dualité lorsqu'on identifie $A_{k}$ à son dual au moyen de $\chi(x y)$. Il s'ensuit que cette mesure n'est autre que le produit $\Pi|d x|_{v}$ lorsque les $|d x|_{v}$ sont choisis comme il vient d'être dit; cela veut dire que, sur chacun des sous-groupes ouverts

$$
\prod_{v \in S} k_{v} \times \prod_{v \notin S} \mathrm{o}_{v}
$$

de $A_{k}$, la mesure en question est égale au produit des mesures $|d x|_{v}$ au sens habituel; on la notera $|d x|_{A}$. On en conclut aussitôt que, si $X_{k}$ est comme plus haut, et si les mesures $|d x|_{v}$ sont définies comme plus haut sur les espaces $X_{v}$ (au moyen d'une base $X^{\circ}$ de $X_{k}$ ), la mesure $|d x|_{A}=\prod|d x|_{v}$ sur $X_{A}$, c'est-à-dire celle qui est égale au produit $\prod|d x|_{v}$ sur chacun des sous-groupes ouverts $X_{S}^{\circ}$ de $X_{A}$ (cf. [14], $\mathrm{n}^{\circ} 29,(29)$ ) n'est autre que la mesure de Tamagawa sur $X_{A}$, pour laquelle $X_{A} / X_{k}$ est de mesure $1 ;|d x|_{A}$ est donc indépendant du choix de $X^{\circ}$, et même de celui de $\chi$.

4. Plus généralement, soit $U$ une variété algébrique sans point multiple, de dimension $n$, rationnelle sur $k$, ou bien une réunion de telles variétés, deux à deux disjointes, en nombre fini. Par une jauge sur $U$, on entendra une forme différentielle $\omega$ de degré $n$ sur $U$, rationnelle sur $k$, partout finie et $\neq 0$; d'après le $\mathrm{n}^{\circ} 3$, une jauge $\omega$ sur $U$ détermine pour tout $v$ une mesure $|\omega|_{v}$ sur $U_{v}$.

Chaque fois que $U$ est une variété affine et $k_{v}$ un corps à valuation discrète, on notera $U_{v}^{\circ}$ l'ensemble des points de $U$ à coordonnées dans $\mathfrak{o}_{v}$; c'est une variété analytique compacte sur $k_{v}$, éventuellement vide. De même, chaque fois que $U$ est représenté comme réunion d'images birégulières $\varphi_{i}\left(U_{i}\right)$ de variétés affines $U_{i}$ en nombre fini, on notera $U_{v}^{\circ}$ la réunion des ensembles $\varphi_{i}\left(\left(U_{i}\right)_{v}^{\circ}\right)$; bien entendu, celle-ci dépend des $U_{i}$ et des $\varphi_{i}$, mais on sait qu'un autre choix des $U_{i}$ et des $\varphi_{i}$ ne modifie $U_{v}^{\circ}$ que pour un nombre fini de places $v$ de $k$ (de sorte que le choix des $U_{i}$ et des $\varphi_{i}$ est sans influence sur les définitions qui vont suivre; cf. [13], Chap. I). La variété adélique $U_{A}$ attachée à $U$ est définie alors comme la réunion, ou plus précisément la limite inductive, des ensembles

$$
U_{S}^{\circ}=\prod_{v \in S} U_{v} \times \prod_{v \in S} U_{v}^{\circ}
$$

On sait (cf. [13], Chap. II) que, pour presque tout $v$, on peut définir une variété $\bar{U}_{v}$ de dimension $n$, sans point multiple, sur le corps fini $\mathfrak{o}_{v} / \mathfrak{p}_{v}$, la « réduction de $U_{v}^{\circ}$ modulo $\mathfrak{p}_{v} "$, telle que l'on ait

$$
\int_{U_{v}^{\circ}}|\omega|_{v}=N\left(\mathfrak{p}_{v}\right)^{-n} \cdot N_{v}
$$

si $N_{v}$ est le nombre de points de $\bar{U}_{v}$ rationnels sur $\mathfrak{o}_{v} / \mathfrak{p}_{v}$. De plus, si on désigne par $m_{v}$ 
la valeur commune des deux membres de cette formule, et si $v$ est le nombre des composantes connexes de $U$, il résulte d'une estimation de Langweil ([10], théorème 1) que $\lim _{v} m_{v}=v$ suivant le filtre des complémentaires de parties finies dans l'ensemble des places de $k$. On en conclut en particulier que $U_{v}^{\circ} \neq \varnothing$ pour presque tout $v$. Cela posé, si, pour toute place $v$ de $k$, on s'est donné un nombre $\lambda_{v}>0$, on dira que $\left(\lambda_{v}\right)$ est un système de facteurs de convergence pour $U$ si le produit $\prod\left(\lambda_{v} m_{v}\right)$ est absolument convergent $\left(^{1}\right)$. Lorsqu'il en est ainsi, on peut considérer, sur chacun des ensembles $U_{S}^{\circ}$, la mesure produit des mesures $\lambda_{v}|\omega|_{v}$, et, sur $U_{A}$, la mesure qui coïncide avec ce produit sur $U_{S}^{\circ}$ dès que $S$ est pris assez grand; cette mesure s'appellera la mesure de Tamagawa déterminée sur $U_{A}$ par la jauge $\omega$ et le système de facteurs $\lambda=\left(\lambda_{v}\right)$ et sera notée $|\lambda \omega|_{A}$; elle ne change pas si on remplace $\omega$ par $c \omega$ avec $c \in k, c \neq 0$.

Considérons en particulier un groupe algébrique connexe $G$, défini sur $k$; sur $G$, il existe toujours des jauges, et toute jauge est " relativement invariante " (c'est-à-dire invariante à un facteur scalaire près) par toute translation à gauche ou à droite. Avec un symbolisme évident, on pourra écrire, si $\omega$ est une jauge sur $G$ :

$$
\omega(a x b)=\chi(a) \chi^{\prime}(b) \omega(x)
$$

quels que soient $a \in G, b \in G ; \chi, \chi^{\prime}$ sont des caractères de $G$, rationnels sur $k$. Alors les jauges $\chi^{-1} \omega, \chi^{\prime-1} \omega$ sont respectivement invariantes à gauche et à droite, et ce sont, à des facteurs constants près, les seules ayant ces propriétés. On a en particulier

$$
\omega\left(a^{-1} x a\right)=\Delta_{G}(a) \omega(x)
$$

avec $\Delta_{G}=\chi^{-1} \chi^{\prime}$; le caractère $\Delta_{G}$ s'appelle le module algébrique (ou parfois simplement le module) de $G$; il est indépendant du choix de $\omega$. Si $\Delta_{G}=1, G$ est dit unimodulaire; c'est le cas pour tout groupe réductif et pour tout groupe unipotent. Si $\omega$ est une jauge sur $G$, et si $\lambda$ est un système de facteurs de convergence pour $G$, les mesures $|\omega|_{v}$ et $|\lambda \omega|_{A}$ sont relativement invariantes, sur les groupes $G_{v}$ et $G_{A}$ respectivement; ce sont des mesures de Haar si $\omega$ est invariante à gauche. Par suite, pour que $G_{A}$ soit unimodulaire (en tant que groupe localement compact), il faut et il suffit que $G$ le soit (en tant que groupe algébrique). On notera 1 le système de facteurs $\left(\lambda_{v}\right)$ pour lequel $\lambda_{v}=1$ quel que soit $v$; d'après un résultat général dû à Serre, c'est un système de facteurs de convergence pour tout groupe semisimple;

(1) Nous aurons souvent à considérer des produits $\Pi a_{v}$ étendus, soit à l'ensemble des places de $k$, soit au complémentaire d'une partie finie de cet ensemble; certains des $a_{v}$ pourront s'annuler. On dira que $\Pi a_{v}$ est absolument convergent s'il existe une partie finie $S$ de l'ensemble des places de $k$ telle que $a_{v}$ soit défini et $\neq 0$ pour tout $v \notin S$ et que le produit partiel $\Pi a_{v}$ restreint aux $v \notin S$ soit absolument convergent au sens usuel. 
pour les groupes classiques, qui interviendront seuls dans la suite de ce travail, ce résultat est démontré dans [13].

Si $G$ est un groupe algébrique non connexe, dont toutes les composantes connexes soient rationnelles sur $k$, on ne peut plus affirmer que toute jauge soit relativement invariante sur $G$; mais il reste vrai que toute jauge relativement invariante à gauche l'est aussi à droite, et qu'il existe de telles jauges; si on se restreint à considérer celles-ci, les faits énoncés plus haut restent valables. On conviendra de dire que $G$ est unimodulaire s'il en est ainsi de la composante connexe $G_{0}$ de l'élément neutre dans $G$. Si $G$ est unimodulaire, $\Delta_{G}$ est un caractère de $G / G_{0}$, et ses valeurs sont des racines de l'unité.

5. Soient $X, Y$ des variétés algébriques et $f$ une application de $X$ dans $Y$; soit $x$ un point simple de $X$ où $f$ soit définie, et supposons que le point $y=f(x)$ soit simple sur $Y$; soient $T_{x}, T_{y}$ les espaces vectoriels respectivement tangents à $X$ en $x$ et à $Y$ en $y$. On dit alors, comme on sait, que $f$ est submersive (ou "de rang maximum ") en $x$ si l'application linéaire de $T_{x}$ dans $T_{y}$ tangente à $f$ en $x$ est surjective.

Supposons $X, Y$ et $f$ rationnels sur $k$; supposons $f$ "génériquement submersive ", c'est-à-dire submersive en un point générique de $X$ sur $k$. Alors l'ensemble $X^{\prime}$ des points de $X$ où $f$ est submersive est $k$-ouvert dans $X$, et $f$ détermine un morphisme partout submersif $f^{\prime}$ de $X^{\prime}$ dans $Y$. De plus, l'application $f^{\prime}$ est $k$-ouverte, de sorte qu'en particulier l'image ensembliste $Y^{\prime}=f^{\prime}\left(X^{\prime}\right)$ de $X^{\prime}$ par $f^{\prime}$ est $k$-ouverte dans $Y$; et, pour tout $y \in Y^{\prime}$, l'image réciproque ensembliste $F_{y}=f^{\prime-1}(\{y\})$ de $y$ dans $X^{\prime}$ par $f^{\prime}$ est une variété sans point multiple, ou une réunion de telles variétés, deux à deux disjointes.

Les hypothèses et notations étant comme ci-dessus, soient de plus $\omega, \eta$ des jauges sur $X^{\prime}$ et sur $Y^{\prime}$; notons $f_{*}^{\prime} \eta$ l'image réciproque de $\eta$ par $f^{\prime}$ sur $X^{\prime}$ (qu'on notera parfois aussi, avec un symbolisme évident, $\eta\left(f^{\prime}(x)\right)$ si $\omega, \eta$ sont notées $\left.\omega(x), \eta(y)\right)$. On vérifie immédiatement qu'il existe un recouvrement $\left(X_{\lambda}\right)_{\lambda \in L}$ de $X^{\prime}$ par des parties $k$-ouvertes $X_{\lambda}$ de $X^{\prime}$ en nombre fini, et, dans chaque $X_{\lambda}$, une forme différentielle $\theta_{\lambda}$ partout finie, rationnelle sur $k$, tels que $\omega$ coïncide avec $\theta_{\lambda} \wedge f_{*}^{\prime} \eta$ dans $X_{\lambda}$ quel que soit $\lambda$. De plus, dans ces conditions, les formes $\theta_{\lambda}, \theta_{\mu}$ induisent une même forme différentielle sur $F_{y} \cap X_{\lambda} \cap X_{\mu}$ quels que soient $y \in Y^{\prime}, \lambda$ et $\mu$; il y a donc sur chaque "fibre " $F_{y}$ une forme différentielle $\theta_{y}$ et une seule qui coïncide sur $F_{y} \cap X_{\lambda}$, quel que soit $\lambda$, avec celle induite par $\theta_{\lambda}$ sur $F_{y} \cap X_{\lambda}$; enfin, on vérifie immédiatement que $\theta_{y}$ est une jauge sur $F_{y}$, rationnelle sur le corps $k(y)$. On écrira, symboliquement, $\theta_{y}=\left(\omega / f_{*}^{\prime} \eta\right)_{y}$, ou encore:

$$
\theta_{y}(x)=\left(\frac{\omega(x)}{\eta\left(f^{\prime}(x)\right)}\right)_{y}
$$

En particulier, prenons pour $X, Y$ et $f$ un groupe algébrique $G$, l'espace homogène 
$H=G / g$ déterminé par $G$ et un sous-groupe $g$ de $G$, et l'application canonique $\pi$ de $G$ sur $H$; celle-ci est partout submersive; les "fibres " $\pi^{-1}(\{y\})$ sont les classes $x g$ suivant $g$ dans $G$; en particulier, si $y_{0}=\pi(e)$, on a $\pi^{-1}\left(\left\{y_{0}\right\}\right)=g$. Supposons qu'il existe sur $H$ une jauge $\eta$ relativement invariante, c'est-à-dire telle que $\eta(a y)=\psi(a) \eta(y)$ quel que soit $a \in G$; et prenons pour $\omega$ une jauge relativement invariante sur $G$ (cf. $\mathrm{n}^{\circ} 4$ ), donc telle que $\omega(a x b)=$ $\chi(a) \chi^{\prime}(b) \omega(x)$. On peut alors, comme plus haut, définir $\theta_{y}=\left(\omega / \pi_{*} \eta\right)_{y}$; en particulier, on vérifie aussitôt que $\theta_{y_{0}}$ est une jauge relativement invariante sur $g$, et plus précisément que l'on a

$$
\theta_{y_{0}}(\alpha \xi \beta)=\chi(\alpha) \psi(\alpha)^{-1} \chi^{\prime}(\beta) \theta_{y_{0}}(\xi)
$$

quels que soient $\alpha \in g, \beta \in g$. Il s'ensuit que, sur $g, \psi$ coïncide avec $\Delta_{g} \Delta_{G}^{-1}$. Ce résultat admet une réciproque (cf. [13], Theorem 2.4.1). On notera en particulier que $\psi=1$ entraîne que $\Delta_{G}$ coïncide avec $\Delta_{g}$ sur $g$; ce sera le cas chaque fois que $g$ est un sous-groupe invariant de $G$, puisqu'alors on peut prendre pour $\eta$ une jauge invariante à gauche sur le groupe quotient $G / g$.

6. Les résultats ci-dessus nous intéressent avant tout dans le cas où $k$ est un corps local $k_{v}$; alors, comme on l'a vu au $\mathrm{n}^{\circ} 4$, les jauges $\omega, \eta$ déterminent des mesures $|\omega|_{v}$, $|\eta|_{v}$ sur $X_{v}^{\prime}, Y_{v}^{\prime}$, et de même $\theta_{y}$ détermine une mesure $\left|\theta_{y}\right|_{v}$ sur $\left(F_{y}\right)_{v}$ chaque fois que $y \in Y_{v}^{\prime}$. L'application du théorème de Lebesgue-Fubini au voisinage de chaque point de $X_{v}^{\prime}$ montre qu'on a, pour toute fonction continue $\Phi$ à support compact sur $X_{v}^{\prime}$ :

$$
\int_{X_{v}^{\prime}} \Phi(x)|\omega(x)|_{v}=\int_{Y_{v}^{\prime}}\left(\int_{\left(F_{y}\right)_{v}} \Phi(z)\left|\theta_{y}(z)\right|_{v}\right)|\eta(y)|_{v},
$$

la fonction à intégrer sur $Y_{v}^{\prime}$ au second membre étant alors continue à support compact; il est immédiat aussi (comme à la fin de la démonstration de la proposition $1, n^{\circ} 2$ ) que la famille des mesures $\left|\theta_{y}\right|_{v}$, de supports respectifs $\left(F_{y}\right)_{v}$, est la seule qui possède ces propriétés. Comme d'habitude en théorie de l'intégration, la formule (7) reste valable pour toute fonction $\Phi$ intégrable sur $X_{v}^{\prime}$, ou encore pour toute fonction $\Phi \geqslant 0$ localement intégrable sur $X_{v}^{\prime}$, par rapport à la mesure $|\omega|_{v}$. Si la variété $X$ elle-même est sans point multiple, et que $\omega$ soit une jauge sur $X$, on ne modifie pas l'intégrale du premier membre de (7) en la prenant $\operatorname{sur} X_{v}$ au lieu de $X_{v}^{\prime}$, puisque $X_{v}-X_{v}^{\prime}$ est réunion de sous-variétés de $X_{v}$ de codimension $\geqslant 1$, en nombre fini, et est donc de mesure nulle; une remarque analogue s'applique à $Y_{v}$ et $Y_{v}^{\prime}$.

Supposons en particulier que $X$ et $Y$ soient des espaces vectoriels; alors $X_{v}, Y_{v}$ sont des espaces vectoriels sur $k_{v}$; supposons que l'application $f$ soit génériquement submersive, partout définie sur $X$, et qu'elle satisfasse à la condition (A) de la proposition $1 \mathrm{du} \mathrm{n}^{\circ} 2$; 
cette proposition permet done de définir, pour tout $y \in Y_{v}$, une mesure tempérée positive $\mu_{y}$, de support contenu dans $f^{-1}(\{y\})$, ayant les propriétés énoncées dans cette proposition. On aura en particulier, pour toute fonction continue $\Phi$ à support compact sur $X_{v}$ :

$$
\int_{X_{v}} \Phi(x)|d x|_{v}=\int_{Y_{v}}\left(\int \Phi d \mu_{y}\right)|d y|_{v},
$$

la fonction à intégrer sur $Y_{v}$ au second membre étant continue. D'autre part, $f$ étant génériquement submersive, on peut, avec les mêmes notations que plus haut, définir une jauge $\theta_{y}=\left(d x / f_{*}^{\prime} d y\right)_{y}$ sur $F_{y}$ quel que soit $y \in Y^{\prime}$, puis une mesure $\left|\theta_{y}\right|_{v}$ sur $\left(F_{y}\right)_{v}$ quel que soit $y \in Y_{v}^{\prime}$, et celle-ci satisfait à (7). La comparaison entre ces formules montre alors que $\mu_{y}$ coïncide avec $\left|\theta_{y}\right|_{v}$ sur $\left(F_{y}\right)_{v}$, ou, ce qui revient au même, sur $f^{-1}(\{y\})_{v}$, au voisinage de tout point de ce dernier ensemble où $f$ est submersive.

7. On va introduire maintenant un type d'intégrale qui joue un grand rôle, non seulement dans la suite de ce travail, mais dans d'autres questions de théorie des nombres (par exemple la " formule de Selberg "). Soient $G$ un groupe algébrique, $X$ une variété sur laquelle opère $G$ par une loi notée $(g, x) \rightarrow g x$; supposons que $G, X$ et cette loi soient définis sur le corps $k$. Soit $\mu$ une mesure positive sur $G_{A} / G_{k}$, ou, ce qui revient au même, une mesure positive sur $G_{A}$, invariante à droite par $G_{k}$. Supposons que $X_{k}$ soit discret dans $X_{A}$, ce qui sera le cas en particulier chaque fois que $X$ est une variété affine. Quelle que soit la fonction $\Phi$ continue à support compact sur $X_{A}$, la fonction

$$
g \rightarrow \sum_{\xi \in X_{k}} \Phi(g \xi)
$$

sera continue sur $G_{A}$, et invariante à droite par $G_{k}$. Posons

$$
I_{\mu}(\Phi)=\int_{G_{A} / G_{k}} \sum_{\xi \in X_{k}} \Phi(g \xi) \cdot d \mu(g)
$$

et supposons que cette intégrale soit absolument convergente pour toute fonction $\Phi$ continue à support compact sur $X_{A}$; il en sera ainsi par exemple chaque fois que $\mu$ est à support compact sur $G_{A} / G_{k}$. Alors $I_{\mu}$ est une mesure positive sur $X_{A}$, dont le support est contenu dans l'adhérence de la réunion des orbites de points de $X_{k} \operatorname{par} G_{A}$.

On peut de plus "décomposer $I_{\mu}$ suivant les orbites " de $G_{k}$ dans $X_{k}$. Soit $\Omega$ l'ensemble de ces dernières orbites; pour chaque $\omega \in \Omega$, choisissons un représentant $\xi_{\omega}$, de sorte que les $\xi_{\omega}$, pour $\omega \in \Omega$, forment un système de représentants de $X_{k}$ pour la relation d'équivalence déterminée par $G_{k}$ dans $X_{k}$. Pour chaque $\omega \in \Omega$, soit $g(\omega)$ le groupe de stabilité de $\xi_{\omega}$ dans $G$. Soit $I_{\mu, \omega}(\Phi)$ l'intégrale analogue à (8), la sommation étant restreinte à $\xi \in \omega$; on a alors 
et l'on peut aussi écrire

$$
I_{\mu}=\sum_{\omega \in \Omega} I_{\mu, \omega}
$$

$$
I_{\mu, \omega}(\Phi)=\int_{G_{A} / g(\omega) k} \Phi\left(g \xi_{\omega}\right) d \mu(g)
$$

Posons $H(\omega)=G / g(\omega)$, et, pour $\omega$ donné dans $\Omega$, notons $\varphi$ le morphisme canonique de $G$ sur $H(\omega)$, en tant que variétés algébriques (c'est-à-dire sur le domaine universel) et $f$ l'application de $G$ dans $X$ donnée par $f(g)=g \xi_{\omega}$. Comme $f$ est constante sur les classes à droite suivant $g(\omega)$, on peut écrire $f=j \circ \varphi$, où $j$ est un morphisme injectif de $H(\omega)$ dans $X$; plus précisément, $j$ détermine un $k$-isomorphisme de $H(\omega)$ sur l'orbite de $\xi_{\omega}$ par $G$ dans $X$; de plus, si on note $X(\omega)$ l'adhérence de cette orbite dans $X$ (au sens de la topologie de Zariski), l'orbite en question est une partie $k$-ouverte de $X(\omega)$, et $X(\omega)$ est une partie $k$ fermée de $X$, et même une sous-variété de $X$ si $G$ est connexe. On déduit de là des applications $\varphi_{A}, j_{A}$ de $G_{A}$ dans $H(\omega)_{A}$ et de $H(\omega)_{A}$ dans $X_{A}$, respectivement; $X(\omega)_{A}$ s'identifie à une partie fermée de $X_{A}$, et $j_{A}$ applique $H(\omega)_{A}$ dans $X(\omega)_{A}$. De plus, $\varphi_{A}$ est constant sur les classes suivant $g(\omega)_{A}$ dans $G_{A}$ et s'écrit donc $\varphi_{A}=\psi \circ \varphi_{1}$, où $\varphi_{1}$ est l'application canonique de $G_{A}$ sur $G_{A} / g(\omega)_{A}$ et $\psi$ une application injective de $G_{A} / g(\omega)_{A}$ dans $H(\omega)_{A}$. Enfin on peut écrire $\varphi_{1}=\varphi^{\prime} \circ \varphi^{\prime \prime}$, où $\varphi^{\prime \prime}$ est l'application canonique de $G_{A}$ sur $G_{A} / g(\omega)_{k}$ et $\varphi^{\prime}$ une application $\operatorname{de} G_{A} / g(\omega)_{l}$ sur $G_{A} / g(\omega)_{A}$.

Par hypothèse, (8) est absolument convergente pour toute fonction $\Phi$ à support com. pact; à plus forte raison, il en est donc de même de (10), et par suite $I_{\mu, \omega}$ est une mesure positive sur $X_{A}$, de support évidemment contenu dans $X(\omega)_{A}$. Si on note $\mu^{\prime}$ la mesure sur $G_{A} / g(\omega)_{k}$ déduite de la mesure $\mu$ sur $G_{A}$ par passage au quotient, $I_{\mu, \omega}$ est l'image de $\mu^{\prime}$ par l'application $j_{A} \circ \psi \circ \varphi^{\prime}$ de $G_{A} / g(\omega)_{k}$ dans $X_{A}$ (cf. Bourbaki, Intégration, Chap. V, $\S 6$ ). Il s'ensuit, comme on sait, que $\mu^{\prime}$ a une image $\mu_{\omega}$ dans $H(\omega)_{A}$ par $\psi \circ \varphi^{\prime}$ et qu'on a

$$
I_{\mu, \omega}(\Phi)=\int_{H(\omega),} \Phi\left(j_{A}(h)\right) d \mu_{\omega}(h)
$$

8. Pour aller plus loin, supposons satisfaite la condition suivante (qui généralise le théorème de Witt) :

(W) Quels que soient l'orbite $\omega \in \Omega$ et le corps $K \supset k$, l'application $\varphi_{K}$ de $G_{K}$ dans $H(\omega)_{K}$ déterminée par l'application canonique $\varphi$ de $G$ sur $H(\omega)$ est surjective.

Il revient au même de dire que, chaque fois que $L \supset K \supset k$ et $\xi \in X_{k}$, tout point de $X_{K}$ qui est dans l'orbite de $\xi$ par $G_{L}$ est dans l'orbite de $\xi \operatorname{par} G_{K}$. Il résulte de là, en particulier, que $H(\omega)=H\left(\omega^{\prime}\right)$ entraîne $\omega=\omega^{\prime}$. 
Nous supposerons aussi que $G_{k}$ est dense dans $G$ au sens de la topologie de Zariski; d'après un résultat de Rosenlicht ([11], p. 44), il en est ainsi en tout cas si $G$ est un groupe linéaire connexe et si $k$ est de caractéristique 0. On sait (cf. [13], pp. 3-4) qu'alors l'application $\psi$, définie ci-dessus, de $G_{A} / g(\omega)_{A}$ dans $H(\omega)_{A}$ est un isomorphisme, de sorte qu'on peut identifier ces espaces au moyen de $\psi$.

Supposons enfin qu'on ait pris pour $\mu$ une mesure relativement invariante sur $G_{A}$, invariante à droite par $G_{k}$, et que la convergence de (8) soit assurée dans ces conditions, quelle que soit $\Phi$ continue à support compact; celle de (10) et de (11) le sera donc aussi. D'après les résultats connus sur les espaces homogènes (cf. [4], $\S 2, \mathrm{n}^{\text {os }} 5-8$ ), on peut calculer le second membre de (10) en intégrant d'abord sur les classes suivant $g(\omega)_{A} / g(\omega)_{k}$, au moyen d'une mesure relativement invariante $v$ convenable, puis sur $G_{A} / g(\omega)_{A}$ au moyen d'une mesure convenable. La fonction à intégrer étant constante sur les classes suivant $g(\omega)_{A}$, il s'ensuit que $g(\omega)_{A} / g(\omega)_{k}$ doit être de mesure finie pour $v$, donc que $\nu$ est bi-invariante et $g(\omega)$ unimodulaire (loc. cit. $\mathrm{n}^{\circ} 6$, corollaire 3). Les mêmes résultats montrent alors que $\mu$ doit être invariante à droite par $g(\omega)_{A}$ et que la mesure $\mu_{\omega}$ définie par (11) est relativement invariante par $G_{A}$; si $\nu$ est prise telle que $g(\omega)_{A} / g(\omega)_{k}$ soit de mesure 1 , on a $\mu_{\omega}=\mu / \nu$; si $\mu$ est invariante à gauche par $G_{A}, \mu_{\omega}$ le sera aussi.

9. Nous allons maintenant donner des critères de convergence pour certaines intégrales de la forme (8). Ayant à nous appuyer sur la théorie de la réduction, nous supposerons dans tout le reste de ce chapitre que le corps k est de caractéristique 0. Nous allons d'abord rappeler les résultats principaux de la théorie en question (cf. [3], [2], [6]).

Un " tore trivial " sur $k$ est un groupe algébrique $T$, produit direct de facteurs isomorphes (sur $k$ ) au groupe multiplicatif à une variable $G_{m}=G L_{1}$. Comme $\left(G_{m}\right)_{A}$ n'est autre que le groupe $I_{k}$ des idèles de $k$, il s'ensuit que, si $T$ est un tore trivial de dimension $d$, $T_{A}$ est isomorphe à $\left(I_{k}\right)^{d}$. Pour $\tau \in \mathbf{R}_{+}^{*}$ (c'est-à-dire $\tau \in \mathbf{R}, \tau>0$ ), notons $a_{\tau}$ l'idèle donné par $a_{\tau}=\left(a_{v}\right)$ avec $a_{v}=\tau$ pour toute place à l'infini $v$ de $k$ (c'est-à-dire chaque fois que $k_{v}$ est isomorphe à $\mathbf{R}$ ou à $\mathbf{C}$ ) et $a_{v}=1$ pour toute autre place; notons $\Theta\left(G_{m}\right)$ le sous-groupe de $I_{k}$ formé des $a_{\tau}$ pour $\tau \in \mathbf{R}_{+}^{*}$; pour $T=\left(G_{m}\right)^{d}$, notons $\Theta(T)$ le sous-groupe $\Theta\left(G_{m}\right)^{d}$ de $T_{A}=\left(I_{k}\right)^{d}$. Alors, pour tout tore trivial $T$, nous noterons $\Theta(T)$ l'image de $\Theta\left(\left(G_{m}\right)^{d}\right)$ pour un isomorphisme de $\left(G_{m}\right)^{d}$ sur $T, d$ étant la dimension de $T$; il est aisé de voir que cette image est indépendante $d u$ choix de l'isomorphisme en question. On pourrait aussi donner comme suit une définition invariante de $\Theta(T)$, au moyen de l'opération $R_{k / \mathbf{q}}$ (la " restriction du corps de base " : cf. [13], Chap. I, $n^{\circ} 1.3$ ); cette opération appliquée à $T$, donne un tore $T^{\prime}=R_{k / \mathbf{Q}}(T)$ défini sur $\mathbf{Q}$, non trivial si $k \neq \mathbf{Q}$, et on peut identifier $T_{A}$ (c'est-à-dire $T_{A_{k}}$ ) avec $T_{A \mathbf{Q}}^{\prime}$. Alors, si $T^{\prime \prime}$ est un tore trivial sur $\mathbf{Q}$ maximal dans $T^{\prime}, \Theta(T)$ est la composante connexe de 1 dans $T_{\infty}^{\prime \prime}$ considéré comme sous-groupe de $T_{A_{\mathbf{Q}}}^{\prime \prime}$, donc de $T_{A_{\mathbf{Q}}}=T_{A}$. 
10. Soit $G$ un groupe algébrique réductif, défini sur $k$; si $G$ n'est pas connexe, on entendra par là que la composante connexe $G_{0}$ de l'élément neutre $e$ dans $G$ est réductive. Soit $T$ un tore trivial (sur $k$ ) maximal dans $G$. Si $\varrho$ est une représentation de $G$, c'est-à-dire un homomorphisme, rationnel sur $k$, de $G$ dans le groupe Aut $(X)$ des automorphismes d'un espace vectoriel $X$, on peut choísir pour $X$ une base formée de "vecteurs propres » de $T$; cela veut dire que, pour chaque vecteur $a$ de cette base, on aura, pour $t \in T, \varrho(t) a=\lambda(t) a$, où $\lambda$ est un caractère de $T$ (c'est-à-dire un homomorphisme de $T$ dans $G_{m}=G L_{1}$, rationnel sur $k$ ); les caractères $\lambda$ qui apparaissent ainsi s'appellent les " poids " de $\varrho$ par rapport à $T$. En particulier, les poids de la représentation adjointe de $G$ dans son algèbre de Lie s'appellent les racines; parmi celles-ci, on peut en choisir un certain nombre, $\alpha_{1}, \ldots, \alpha_{r}$ de manière que toute racine $\alpha$ puisse se mettre d'une manière et d'une seule sous la forme $\alpha=\prod \alpha_{i}^{n_{i}}$ avec des exposants $n_{i}$ entiers, et que, pour toute racine $\alpha$, les $n_{i}$ soient tous $\geqslant 0$ ou bien tous $\leqslant 0$; on écrira $\alpha \succ 1$ dans le premier cas, $\alpha \prec 1$ dans le second. Les vecteurs propres relatifs aux racines $\alpha \succ 1$ forment l'algèbre de Lie d'un sous-groupe connexe $P$ de $G$ (" groupe parabolique minimal " relativement à $k$ ) qui contient $T ; \Delta_{P}$ étant comme d'habitude le module (algébrique) de $P$, la restriction de $\Delta_{P}^{-1}$ à $T$ est le produit de toutes les racines $\alpha \succ 1$. L'espace homogène $G_{0} / P$ est isomorphe à une variété projective; comme d'autre part on démontre que $\left(G_{0}\right)_{A} / P_{A}$ est isomorphe à $\left(G_{0} / P\right)_{A}$, il s'ensuit que c'est un espace compact; puisque $G_{A} /\left(G_{0}\right)_{A}$ est compact (cf. [2], $\mathrm{n}^{0} 1.9$ ), il en est donc de même de $G_{A} / P_{A}$, de sorte qu'il existe une partie compacte $C_{1}$ de $G_{A}$ telle que $G_{A}=C_{1} \cdot P_{A}$.

On notera $P_{A}^{\circ}$ le sous-groupe de $P_{A}$, intersection des noyaux des homomorphismes $p \rightarrow|\chi(p)|_{A}$ de $P_{A}$ dans $\mathbf{R}_{+}^{*}$ lorsqu'on prend pour $\chi$ tous les caractères de $P$ (rationnels sur $k$ ); bien entendu, $|a|_{A}$, pour tout $a \in I_{k}$, désigne le module de l'automorphisme $x \rightarrow a x$ de $A_{k}$. Alors $P_{A}^{\circ} / P_{k}$ est compact, de sorte qu'il existe une partie compacte $C_{2}$ de $P_{A}^{\circ}$ telle que $P_{A}^{\circ}=C_{2} \cdot P_{k}$. De plus, le groupe des caractères de $P$ induit sur $T$ un sous-groupe d'indice fini du groupe des caractères de $T$; on en conclut aussitôt que l'homomorphisme canonique $\operatorname{de} P_{A}$ sur $P_{A} / P_{A}^{\circ}$ induit sur $\Theta(T)$ un isomorphisme de $\Theta(T)$ sur $P_{A} / P_{A}^{\circ} ; P_{A}$ est donc produit semidirect de $\Theta(T)$ et de $P_{A}^{\circ}$; on peut écrire $P_{A}=\Theta(T) \cdot P_{A}^{\circ}$, et l'application $\left(\theta, p_{0}\right) \rightarrow \theta p_{0}$ est un homéomorphisme de $\Theta(T) \times P_{A}^{\circ} \operatorname{sur} P_{A}$.

Comme le module $\left|\Delta_{P}(p)\right|_{A}$ de $P_{A}$ (en tant que groupe localement compact) prend la valeur 1 sur le sous-groupe invariant $P_{A}^{\circ}$ de $P_{A}$, celui-ci est unimodulaire. Soient $d \theta$, $d p_{0}$ des mesures de Haar sur $\Theta(T)$ et sur $P_{A}^{\circ}$, respectivement; l'image de la mesure produit $d \theta d p_{0}$ par l'homéomorphisme $\left(\theta, p_{0}\right) \rightarrow \theta p_{0}$ de $\Theta(T) \times P_{A}^{\circ}$ sur $P_{A}$ sera alors une mesure de Haar sur $P_{A}$, qu'on notera aussi $d \theta d p_{0}$. Alors la mesure $d^{\prime} p=\left|\Delta_{P}(\theta)\right|_{A}^{-1} d \theta d p_{0}$ sera invariante à droite sur $P_{A}$.

Enfin, on désignera par $T_{A}^{+}$l'ensemble des $t \in T_{A}$ tels que l'on ait $|\alpha(t)|_{A} \leqslant 1$ pour toute 2-652922. Acta mathematica. 113. Imprimé le 26 février 1965. 
racine $\alpha>1$, et on posera $\Theta^{+}=\Theta(T) \cap T_{A}^{+}$et $P_{A}^{+}=\Theta^{+} \cdot P_{A}^{\circ}=T_{A}^{+} \cdot P_{A}^{\circ}$. On sait ([6], lemme 1, p. 17) que, si $C$ est une partie compacte de $P_{A}$, la réunion des $\theta C \theta^{-1}$, pour $\theta \in \Theta^{+}$, est relativement compacte dans $P_{A}$; en particulier, si $C_{2}$ est choisi comme il a été dit, c'est-à-dire compact et tel que $P_{A}^{\circ}=C_{2} \cdot P_{k}$, on désignera par $C_{3}$ l'adhérence de la réunion des $\theta C_{2} \theta^{-1}$ pour $\theta \in \Theta^{+}$; c'est une partie compacte de $P_{A}^{\circ}$.

Avec ces notations, le résultat principal de la théorie de la réduction s'énonce comme suit : il existe une partie compacte $C$ de $G_{A}$ telle que $G_{A}=C \cdot P_{A}^{+} \cdot G_{k}$. Si l'on pose $N=C \cdot P_{A}^{+}$, cela entraîne que l'image de $N / P_{k}$, dans l'application canonique de $G_{A} / P_{k}$ sur $G_{A} / G_{k}$, est $G_{A} / G_{k}$

11. Soit maintenant $d g$ une mesure de Haar sur $G_{A}$. Pour étudier la convergence des intégrales $\operatorname{sur} G_{A} / G_{k}$, on s'appuyera sur le lemme suivant :

LEMME 4. Soient $G, \Theta^{+}$comme il a été dit au $n^{\circ}$ 10. Alors il existe une partie compacte $C_{0}$ de $G_{A}$ et une constante $\gamma>0$ telles que l'on ait

$$
\int_{G_{A} / G_{k}}|F(g)| d g \leqslant \gamma \int_{\theta^{+}} F_{0}(\theta) \cdot\left|\Delta_{P}(\theta)\right|_{A}^{-1} d \theta
$$

chaque fois que $F, F_{0}$ sont des fonctions, localement intégrables sur $G_{A} / G_{k}$ et sur $\Theta^{+}$respectivement, telles que $|F(c \theta)| \leqslant F_{0}(\theta)$ quels que soient $c \in C_{0}$ et $\theta \in \Theta^{+}$.

Désignons par $I$ le premier membre de (12) et par $\varphi_{N}$ la fonction caractéristique de l'ensemble $N=C \cdot P_{A}^{+}$introduit à la fin du $n^{\circ} 10$. On aura

$$
I \leqslant \int_{N / P_{\boldsymbol{k}}}|F(g)| d g=\int_{G_{\boldsymbol{A}} / \boldsymbol{P}_{\mathbf{k}}}|F(g)| \varphi_{N}(g) d g .
$$

On va transformer cette dernière intégrale au moyen de la théorie des mesures quasiinvariantes dans les espaces homogènes (v. [4], $§ 2, n^{\text {os }} 5-8$ ). D'après celle-ci, on peut construire $\operatorname{sur} G_{A}$ une fonction continue $h$, partout $>0$, telle qu'on ait $h(g p)=h(g)\left|\Delta_{P}(p)\right|_{A}$ quels que soient $g \in G_{A}, p \in P_{A}$, puis sur $G_{A} / P_{A}$ une mesure positive $\lambda$ telle que l'on ait, pour toute fonction $f \geqslant 0$ localement intégrable sur $G_{A} / P_{k}$ :

$$
\int_{G_{\boldsymbol{A}} / \boldsymbol{P}_{\boldsymbol{k}}} f(g) d g=\int_{G_{\boldsymbol{A}} / P_{\boldsymbol{A}}}\left(h(g) \int_{\boldsymbol{P}_{\boldsymbol{A}} / \boldsymbol{P}_{\boldsymbol{k}}} f(g p) d^{\prime} p\right) d \lambda(g)
$$

où $g$ est l'image canonique de $g \in G_{A}$ dans $G_{A} / P_{A}$, et où $d^{\prime} p$ est la mesure invariante à droite dans $P_{A}$ qu'on a introduite au $\mathrm{n}^{\circ} 10$; la condition imposée à $h$ entraîne que la fonction à intégrer sur $G_{A} / P_{A}$, qui est écrite comme fonction de $g \in G_{A}$, est constante sur les classes suivant $P_{A}$ et peut donc être considérée comme fonction sur $G_{A} / P_{A}$. Appliquant cette formule au dernier membre de (13), on obtient 


$$
I \leqslant \int_{G_{A} / P_{A}} \psi(g) d \lambda(\dot{g})
$$

où $\psi$ est la fonction définie par la formule

$$
\psi(g)=h(g) \int_{P_{A} / P_{k}}|F(g p)| \varphi_{N}(g p) d^{\prime} p
$$

$\mathrm{Au}$ second membre de celle-ci, on peut prendre pour $g$ n'importe quel représentant de la classe $g$ suivant $P_{A}$ dans $G_{A}$; comme $G_{A}=C_{1} \cdot P_{A}$, on peut donc supposer qu'on a pris $g \in C_{1}$. Mais alors on a $\varphi_{N}(g p)=0$ quand $p \notin C_{1}^{-1} N$. Posons :

$$
Q=C_{1}^{-1} N \cap P_{A}=\left(C_{1}^{-1} C \cap P_{A}\right) \cdot P_{A}^{+}=\left(C_{1}^{-1} C \cap P_{A}\right) \cdot \Theta^{+} \cdot P_{A}^{\circ}
$$

soit aussi $\gamma_{1}$ la borne supérieure de $h$ sur $C_{1}$, et soit $F_{1}(p)$, pour tout $p \in P_{A}$, la borne supérieure de $|F(g p)|$ pour $g \in C_{1}$. On aura done :

$$
\psi(g) \leqslant \gamma_{1} \int_{Q / P_{k}}|F(g p)| d^{\prime} p \leqslant \gamma_{1} \int_{Q / P_{k}} F_{1}^{\prime}(p) d^{\prime} p
$$

et par conséquent, puisque $G_{A} / P_{A}$ est compact:

$$
I \leqslant \gamma_{2} \int_{Q / P_{k}} F_{1}(p) d^{\prime} p
$$

pourvu que la constante $\gamma_{2}$ soit convenablement choisie.

Comme au $\mathrm{n}^{\circ} 10$, identifions $P_{A} / P_{.4}^{\circ}$ avec $\Theta(T)$; il est immédiat d'ailleurs que toute partie compacte de $\Theta(T)$ est contenue dans un ensemble de la forme $\theta_{0} \Theta^{+}$, avec $\theta_{0} \in \Theta(T)$; appliquant cette remarque à l'image de $C_{1}^{-1} C \cap P_{A}$ dans $P_{A} / P_{A}^{\circ}=\Theta(T)$, on en conclut qu'il y a $\theta_{0} \in \Theta(T)$ tel que $Q$ soit contenu dans $\theta_{0} \Theta^{+} \cdot P_{A}^{\circ}$. Comme d'autre part on a $P_{A}^{\circ}=$ $C_{2} \cdot P_{k}$, on obtient $Q \subset \theta_{0} \Theta^{+} \cdot C_{2} \cdot P_{k}$, et par suite

$$
I \leqslant \gamma_{2} \int_{\theta_{0} \Theta^{+}, C_{2}} F_{1}(p) d^{\prime} p
$$

Comme $d^{\prime} p$ a été défini au $n^{\circ} 10$ au moyen de $d^{\prime} p=\left|\Delta_{P}(\theta)\right|_{A}^{-1} d \theta d p_{0}$, cela s'écrit aussi

$$
I \leqslant \gamma_{2} \int_{C_{2}}\left(\int_{\Theta^{+}} F_{1}\left(\theta_{0} \theta p_{0}\right) \cdot\left|\Delta_{P}\left(\theta_{0} \theta\right)\right|_{A}^{-1} d \theta\right) d p_{0} .
$$

Mais, comme on l'a vu au n $\mathrm{n}^{\circ} 10$, il y a une partie compacte $C_{3}$ de $P_{A}^{\circ}$ telle que $\theta C_{2} \theta^{-1} \subset C_{3}$, donc aussi $\theta_{0} \theta C_{2} \subset \theta_{0} C_{3} \theta$, quel que soit $\theta \in \Theta^{+}$. Si donc on note $F_{2}(\theta)$, pour tout $\theta \in \Theta^{+}$, la borne supérieure de $F_{1}(p \theta)$ pour $p \in \theta_{n} C_{2}$, on obtient 


$$
I \leqslant \gamma \int_{\Theta^{+}} F_{2}(\theta) \cdot\left|\Delta_{P}(\theta)\right|_{A}^{-1} d \theta
$$

pourvu que la constante $\gamma$ soit convenablement choisie. Il s'ensuit que l'assertion du lemme est vérifiée si on prend $C=C_{1} \theta_{0} C_{3}$.

Dans l'énoncé du lemme 4, on pourrait, sans rien changer en substance, substituer $T_{A}^{+} / T_{k}$ à $\Theta^{+}$; on obtiendrait ainsi un énoncé équivalent, moins commode à utiliser directement, mais qui aurait l'avantage de ne pas faire jouer un rôle privilégié aux places à l'infini (et vraisemblablement, par suite, de rester valable en toute caractéristique).

12. Nous allons maintenant appliquer le lemme 4 aux intégrales de la forme (8) considérée aux $\mathrm{n}^{\text {os }} 7-8$, en nous bornant au cas où $\mu$ est une mesure de Haar sur $G_{A}$ et $X$ un espace affine sur lequel $G$ opère au moyen d'une représentation $\varrho$ de $G$ dans Aut $(X)$. Les notations restant les mêmes que dans les $n^{\text {os }} 10-11$, nous désignerons par $\Lambda$ le groupe des caractères de $T$, et, pour tout $\lambda \in \Lambda$, nous noterons $m_{\lambda}$ la dimension sur $k$ de l'espace des vecteurs $a \in X_{k}$ tels que $\varrho(t) a=\lambda(t) a$ quel que soit $t \in T$. Les caractères $\lambda$ de $T$ pour lesquels $m_{\lambda}>0$ sont les poids de la représentation $\varrho ; m_{\lambda}$ est la multiplicité du poids $\lambda$.

L тм Е 5. Soient $G, T, \Theta^{+}$comme il a été dit au $n^{\circ} 10$; soit $\varrho$ une représentation de $G$ dans le groupe $\operatorname{Aut}(X)$ des automorphismes d'un espace affine $X$; pour chaque caractère $\lambda$ de $T$, soit $m_{\lambda}$ sa multiplicité en tant que poids de $\varrho$. Alors l'intégrale

$$
I(\Phi)=\int_{G_{A} / G_{k}} \sum_{\xi \in X_{k}} \Phi(\varrho(g) \xi) \cdot d g
$$

est absolument convergente pour toute fonction $\Phi \in S\left(X_{A}\right)$ chaque fois que l'intégrale

$$
\int_{\Theta^{+}} \prod_{\lambda} \sup \left(1,|\lambda(\theta)|_{A}^{-m_{\lambda}}\right) \cdot\left|\Delta_{P}(\theta)\right|_{A}^{-1} d \theta
$$

est convergente; et, quand il en est ainsi, (14) définit une mesure positive tempérée 1.

Si $I(\Phi)$ est absolument convergente pour toute fonction $\Phi \in S\left(X_{A}\right)$, le lemme 5 de [14], $n^{\circ} 41$, montre qu'elle l'est uniformément sur toute partie compacte de $S\left(X_{A}\right)$, d'où il résulte bien, d'après le lemme $2 \mathrm{du} \mathrm{n}^{\circ} 2$, que $I$ est une distribution tempérée, donc une mesure positive tempérée. Soit maintenant $C_{0}$ une partie compacte de $G_{A}$ ayant la propriété énoncée dans le lemme $4 \mathrm{du} \mathrm{n}^{\circ} 11$ ci-dessus. Pour $\Phi$ donnée dans $S\left(X_{A}\right)$, il existe, d'après le lemme 5 de [14], $n^{\circ} 41$, une fonction $\Phi_{1} \in S\left(X_{A}\right)$ telle que l'on ait

$$
|\Phi(\varrho(c) x)| \leqslant \Phi_{1}(x)
$$


quels que soient $c \in C_{0}$ et $x \in X_{A}$. L'application du lemme 4 à (14) montre alors que $I(\Phi)$ est absolument convergente pourvu qu'il en soit ainsi de l'intégrale

$$
I_{1}=\int_{\Theta^{+}} \sum_{\xi \in X_{k}} \Phi_{1}(\varrho(\theta) \xi) \cdot\left|\Delta_{P}(\theta)\right|_{A}^{-1} d \theta
$$

Désignons par $X_{\infty}$ le produit $\prod X_{v}$ étendu aux places à l'infini de $k$ (ou, ce qui revient au même, posons $X_{\infty}=X_{k} \otimes \mathbf{R}$, le produit tensoriel étant pris sur $\mathbf{Q}$; cf. [14], $\mathrm{n}^{\circ} 39$ ); on pourra écrire $X_{A}=X_{\infty} \times X^{\prime}$, où $X^{\prime}$ est défini comme $X_{A}$ mais au moyen des places $v$ de $k$ pour lesquelles $k_{v}$ est à valuation discrète. Compte tenu de la définition de $S\left(X_{A}\right)$ (cf. [14], $\mathrm{n}^{\text {os }}$ 29 et 39 ), on peut supposer que $\Phi_{1}$ a été choisi de la forme

$$
\Phi_{1}(x)=\Phi_{\infty}\left(x_{\infty}\right) \Phi^{\prime}\left(x^{\prime}\right)
$$

où $x_{\infty}, x^{\prime}$ sont les projections de $x \in X_{A}$ sur $X_{\infty}$ et $X^{\prime}$, avec $\Phi_{\infty} \in S\left(X_{\infty}\right)$, $\Phi^{\prime}$ étant la fonction caractéristique d'un sous-groupe ouvert compact de $X^{\prime}$. L'ensemble des $\xi \in X_{k} \operatorname{dont}$ la projection sur $X^{\prime}$ appartient au support de $\Phi^{\prime}$ est alors un réseau $L \subset X_{k}$, c'est-à-dire un groupe abélien de type fini tel que $\mathbf{Q} L=X_{k}$, et $I_{1}$ s'écrit :

$$
I_{1}=\int_{\Theta^{+}} \sum_{\xi \in L} \Phi_{\infty}(\varrho(\theta) \xi) \cdot\left|\Delta_{P}(\theta)\right|_{A}^{-1} d \theta .
$$

Pour tout poids $\lambda$ de $\varrho$, soit $X_{\lambda}$ le sous-espace de $X_{k}$, de dimension $m_{\lambda}$ sur $k$, formé des vecteurs propres de poids $\lambda$, c'est-à-dire des vecteurs $a$ tels que $\varrho(t) a=\lambda(t) a$ pour $t \in T ; X_{k}$ est somme directe $\operatorname{des} X_{\lambda}$. Soit $d=[k: \mathbf{Q}]$; soit

$$
\left(a_{\lambda i}\right)_{1 \leqslant i \leqslant m_{\lambda} d}
$$

une base de $X_{\lambda}$ sur $\mathbf{Q}$; en remplaçant au besoin les $a_{\lambda i}$ par $N^{-1} a_{\lambda_{i}}$, où $N$ est un entier convenable, on peut supposer que le réseau $L$ est contenu dans le sous-groupe de $X_{k}$ engendré par l'ensemble des $a_{\lambda i}$. Les $a_{\lambda i}$ forment aussi une base de $X_{\infty}$ sur $\mathbf{R}$; pour $x_{\infty} \in X_{\infty}$, on pourra donc écrire

$$
x_{\infty}=\sum_{\lambda, i} x_{\lambda i} a_{\lambda i}
$$

avec $x_{\lambda_{i}} \in \mathbf{R}$; alors, si $\alpha>1$, il y aura une constante $C$ telle que l'on ait

$$
\Phi_{\infty}\left(x_{\infty}\right) \leqslant C \prod_{\lambda, i}\left(1+\left|x_{\lambda i}\right|^{\alpha}\right)^{-1}
$$

D'autre part, on aura dans ces conditions

$$
\varrho(\theta) x_{\infty}=\sum_{\lambda, i} \lambda(\theta) x_{\lambda i} a_{\lambda i}
$$

et, si $x_{\infty}$ est la projection sur $X_{\infty}$ d'un élément $\xi$ du réseau $L$, tous les $x_{\lambda_{i}}$ sont entiers en vertu du choix des bases $\left(a_{\lambda i}\right)$. On a donc 


$$
\begin{aligned}
\sum_{\xi \in L} \Phi_{\infty}(\varrho(\theta) \xi) & \leqslant C \prod_{\lambda}\left(\sum_{n=-\infty}^{+\infty} \frac{1}{1+\lambda(\theta)^{\alpha}|n|^{\alpha}}\right)^{m_{\lambda} d} \\
& \leqslant C^{\prime} \prod_{\lambda} \sup \left(1, \lambda(\theta)^{-1}\right)^{m_{\lambda} d}
\end{aligned}
$$

où $C^{\prime}$ est une constante convenable. Si on observe qu'on a $|\lambda(\theta)|_{A}=\lambda(\theta)^{d}$ pour tout caractère $\lambda$ de $T$ et pour tout $\theta \in \Theta$, on voit que cela donne bien la conclusion annoncée.

Si on applique le lemme 5 au cas où $X=\{0\}$, et où par conséquent $\varrho$ est la représentation triviale de $G$, on retrouve le résultat de Borel et Harishchandra, d'après lequel $G_{A} / G_{k}$ est de mesure finie quand le centre de $G$ ne contient aucun tore trivial autre que $\{1\}$.

Il y aurait naturellement intérêt à examiner si la condition de convergence de (14), donnée par le lemme 5, est nécessaire aussi bien que suffisante.

13. Enfin, dans l'application des résultats ci-dessus, on aura à se servir du lemme suivant, où on a de nouveau désigné par $a_{\tau}$, pour $\tau \in \mathbf{R}_{+}^{*}$, l'idèle $\left(a_{v}\right)$ donné par $a_{v}=\tau$ pour toute place à l'infini $v$ de $k$, et $a_{v}=I$ pour toute autre place $v$ :

Lемм 6 6. Soient $\left(X_{k}^{(\alpha)}\right)_{1 \leqslant \alpha \leqslant n}$ et $Y_{k}$ des espaces vectoriels sur $k$; soit $X_{k}=\prod_{\alpha} X_{k}^{(\alpha)}$, et soit $p$ un morphisme de $X$ dans $Y$, rationnel sur $k$ et tel que $p\left(0, x^{(2)}, \ldots, x^{(n)}\right)=0$ quels que soient $x^{(2)}, \ldots, x^{(n)}$. Soit de plus $C_{0}$ une partie compacte de $S\left(X_{A}\right)$, et soit $N \geqslant 0$. Alors il existe une fonction $\Phi_{0} \in \boldsymbol{S}\left(X_{A}\right)$ telle que l'on ait

$$
\left|\tau_{1}^{N} \Phi\left(a_{\tau_{1}} x^{(1)}, \ldots, a_{\tau_{n}} x^{(n)}\right)\right| \leqslant \Phi_{0}(x)
$$

chaque fois que $\Phi \in C_{0}, \tau_{1} \geqslant 1, \ldots, \tau_{n} \geqslant 1, x=\left(x^{(1)}, \ldots, x^{(n)}\right) \in X_{A}, p(x) \in Y_{k}$ et $p(x) \neq 0$.

Rappelons qu'un " morphisme " d'un espace affine dans un autre n'est pas autre chose qu'une application polynomiale; l'hypothèse faite sur $p$ revient donc à dire que, si on choisit des bases de $X_{k}$ et de $Y_{k}$ sur $k$, les coordonnées de $p(x)$ s'expriment comme polynomes à coefficients dans $k$ au moyen de celles de $x$. On désignera par $D$ le plus grand des degrés de ces polynomes. D'autre part, écrivons $X_{A}=X_{\infty} \times X^{\prime}$, où $X_{\infty}$ et $X^{\prime}$ sont définis comme au $\mathrm{n}^{\circ} 12$ dans la démonstration du lemme 5 , et de même $X_{A}^{(\alpha)}=X_{\infty}^{(\alpha)} \times X^{\prime(\alpha)}$ et $Y_{A}=Y_{\infty} \times Y^{\prime} ; p$ détermine d'une manière évidente des applications de $X_{\infty}$ dans $Y_{\infty}$ et de $X^{\prime}$ dans $Y^{\prime}$. Choisissons des bases des $X_{\infty}^{(\alpha)}$ et de $Y_{\infty}$ sur $\mathbf{R}$, et, pour $x_{\infty}^{(\alpha)} \in X_{\infty}^{(\alpha)}$ (resp. $y_{\infty} \in Y_{\infty}$ ), désignons par $r_{\alpha}\left(x_{\infty}^{(\alpha)}\right)$ (resp. $s\left(y_{\infty}\right)$ ) la somme des carrés des coordonnées de $x_{\infty}^{(\alpha)}$ (resp. de $y_{\infty}$ ) par rapport à ces bases. Posons encore, pour $x_{\infty}=\left(x_{\infty}^{(1)}, \ldots, x_{\infty}^{(n)}\right) \in X_{\infty}$ :

$$
r^{\prime}\left(x_{\infty}\right)=\sum_{\alpha \geqslant 2} r_{\alpha}\left(x_{\infty}^{(\alpha)}\right), \quad r\left(x_{\infty}\right)=r_{1}\left(x_{\infty}^{(1)}\right)+r^{\prime}\left(x_{\infty}\right)
$$


Puisque $p(x)$ s'annule chaque fois que $x^{(1)}=0$, il $\mathrm{y}$ a une constante $C>0$ telle que l'on ait, quel que soit $x_{\infty} \in X_{\infty}$ :

$$
s\left(p\left(x_{\infty}\right)\right) \leqslant C \cdot r_{1}\left(x_{\infty}^{(1)}\right) \cdot r\left(x_{\infty}\right)^{D-1}
$$

et par conséquent, pour $\tau_{1} \geqslant \mathbf{1}$ :

$$
s\left(p\left(x_{\infty}\right)\right) \leqslant C \tau_{1}^{-2}\left(\tau_{1}^{2} r_{1}\left(x_{\infty}^{(1)}\right)+r^{\prime}\left(x_{\infty}\right)\right)^{D} .
$$

Convenons de poser, pour $\tau=\left(\tau_{1}, \ldots, \tau_{n}\right), \tau_{1}>0, \ldots, \tau_{n}>0$, et $x_{\infty} \in X_{\infty}$ :

$$
\tau x_{\infty}=\left(\tau_{1} x_{\infty}^{(1)}, \ldots, \tau_{n} x_{\infty}^{(n)}\right)
$$

l'inégalité qu'on vient d'obtenir montre qu'on aura à plus forte raison, chaque fois que $\tau_{1} \geqslant 1, \ldots, \tau_{n} \geqslant 1$ :

$$
s\left(p\left(x_{\infty}\right)\right) \leqslant C \tau_{1}^{-2} r\left(\tau x_{\infty}\right)^{D}
$$

Appliquons maintenant, comme au $\mathrm{n}^{\circ} 12$, le lemme 5 de [14], $\mathrm{n}^{\circ} 41$; il montre qu'on peut choisir $\Phi_{1} \in S\left(X_{A}\right)$ telle que $|\Phi(x)| \leqslant \Phi_{1}(x)$ quels que soient $\Phi \in C_{0}$ et $x=\left(x_{\infty}, x^{\prime}\right) \in X_{A}$, et même qu'on peut supposer $\Phi_{1}$ de la forme

$$
\Phi_{1}(x)=\Phi_{\infty}\left(x_{\infty}\right) \Phi^{\prime}\left(x^{\prime}\right)
$$

où $\Phi_{\infty} \in S\left(X_{\infty}\right)$ et où $\Phi^{\prime}$ est la fonction caractéristique d'un sous-groupe ouvert compact de $X^{\prime}$. Soit $E$ l'ensemble des points $x=\left\langle x_{\infty}, x^{\prime}\right)$ de $X_{A}$ tels que $p(x) \in Y_{k}, p(x) \neq 0$ et $\Phi^{\prime}\left(x^{\prime}\right) \neq 0$; on va montrer que, sur $E, s\left(p\left(x_{\infty}\right)\right)$ a une borne inférieure $\varepsilon>0$. En effet, s'il n'en était pas ainsi, il y aurait une suite de points $x_{v}=\left(x_{\nu \infty}, x_{v}^{\prime}\right)$ de $E$ telle que la suite $p\left(x_{\nu \infty}\right)$ tende vers 0 dans $Y_{\infty}$. Comme le support de $\Phi^{\prime}$ est compact, on peut supposer en même temps que la suite $x_{v}^{\prime}$ tende vers une limite $\bar{x}^{\prime}$, donc que $p\left(x_{v}^{\prime}\right)$ tende vers $p\left(\bar{x}^{\prime}\right)$. Mais alors la suite des points $y_{v}=p\left(x_{v}\right)$ tend vers une limite $\bar{y}$ dans $Y_{A}$, pour laquelle on a $\bar{y}_{\infty}=0$, c'est-à-dire $\bar{y}_{v}=0$ pour toute place à l'infini $v$ de $k$. Comme les $y_{v}$ appartiennent à $Y_{k}-\{0\}$, qui est discret dans $Y_{A}$, on a $\bar{y} \in Y_{k}, \bar{y} \neq 0$, donc $\bar{y}_{v} \neq 0$ quel que soit $v$, d'où contradiction. Tenant compte de l'inégalité démontrée plus haut, on a donc, pour $x \in E, \tau_{1} \geqslant 1, \ldots, \tau_{n} \geqslant 1$ :

avec $C^{\prime}=(C / \varepsilon)^{\frac{1}{2}}$.

$$
\tau_{1} \leqslant C^{\prime} r\left(\tau x_{\infty}\right)^{D / 2}
$$

Posons maintenant, pour tout $i \geqslant 0$ :

$$
a_{i}=\sup _{x_{\infty} \in X_{\infty}}\left(r\left(x_{\infty}\right)^{i} \Phi_{\infty}\left(x_{\infty}\right)\right)
$$

Soit $M$ un entier $\geqslant N D / 2$. D'après le lemme 4 de [14], $\mathrm{n}^{\circ} 41$, il y a $\varphi \in S(R)$ tel que l'on ait, pour tout $r \in \mathbf{R}$ : 


$$
\varphi(r) \geqslant \inf _{i \geqslant 0}\left(a_{M+i}|r|^{-i}\right)
$$

Pour $x$ et $\tau$ comme ci-dessus, on aura donc, quel que soit $i \geqslant 0$ :

$$
\begin{aligned}
\tau_{1}^{2 M / D} \Phi_{\infty}\left(\tau x_{\infty}\right) & \leqslant C^{2 M / D} r\left(\tau x_{\infty}\right)^{M} \Phi_{\infty}\left(\tau x_{\infty}\right) \\
& \leqslant C^{2 M / D} a_{M+i} r\left(\tau x_{\infty}\right)^{-i} \leqslant C^{2 M / D} a_{M+i} r\left(x_{\infty}\right)^{-i}
\end{aligned}
$$

et par suite, à plus forte raison

$$
\tau_{1}^{N} \Phi_{\infty}\left(\tau x_{\infty}\right) \leqslant C^{2 M / D} \varphi\left(r\left(x_{\infty}\right)\right) .
$$

On satisfera donc aux conditions du lemme en posant

$$
\Phi_{0}(x)=C^{2 M / D} \varphi\left(r\left(x_{\infty}\right)\right) \Phi^{\prime}\left(x^{\prime}\right) .
$$

\section{Préliminaires algébriques}

Dans ce Chapitre, on supposera une fois pour toutes que le corps de base $k$ n'est pas de caractéristique 2. Chaque fois qu'il y aura lieu de faire des hypothèses supplémentaires sur $k$, cela sera mentionné explicitement.

14. Reprenant les hypothèses et les notations de [14], $\mathrm{n}^{\circ} 49$, nous considérons, sur le corps de base $k$, une algèbre $\mathcal{A}$ munie d'une involution $\iota$ et d'une fonction trace $\tau$, ainsi que des modules à gauche sur $\mathcal{A}$. Eventuellement, on pourra avoir à "étendre " ces données à un corps $K$ contenant $k$; cela veut dire qu'on prend le produit tensoriel $\mathcal{A}_{K}$ de $\mathcal{A}$ et de $K$ sur $k$, en le munissant de la structure d'algèbre évidente sur $K$ et de l'involution et de la fonction trace qui se déduisent " naturellement " de $\iota$ et $\tau$; par abus de notation, on les notera encore $\iota$ et $\tau$ (au lieu de $\iota_{K}, \tau_{K}$ ). De même, l'extension par $K$ d'un $\mathcal{A}$-module à gauche $X$ donnera un $\mathcal{A}_{K}$-module à gauche $X_{K}$; etc.

Du fait que $k$ n'est pas de caractéristique 2, certains résultats de [14], Chap. V, peuvent être précisés ou énoncés plus simplement. Par exemple, on a déjà observé au no 31 de [14] que $P s(X)$ est alors isomorphe au groupe symplectique. Plus généralement, notons $S p(X / \mathcal{A})$ le groupe des automorphismes $\sigma \mathrm{du} \mathcal{A}$-module à gauche $X \oplus X^{*}$ qui satisfont à $\sigma \sigma^{I}=\mathbf{I}, \sigma^{\mathrm{I}}$ étant défini comme d'habitude par

$$
\sigma=\left(\begin{array}{cc}
\alpha & \beta \\
\gamma & \delta
\end{array}\right), \quad \sigma^{\mathrm{I}}=\left(\begin{array}{rr}
\delta^{*} & -\beta^{*} \\
-\gamma^{*} & \alpha^{*}
\end{array}\right) .
$$

Alors, si $\sigma \in S p(X / \mathcal{A})$, il y a un élément $f$ et un seul de $Q(X / \mathcal{A})$ tel que $(\sigma, f)$ appartienne à $P s(X / \mathcal{A})$; un calcul facile montre que $f$ est donné par les formules 


$$
\begin{aligned}
& f\left(x, x^{*}\right)=\frac{1}{2} \tau\left(F\left(x, x^{*} ; x, x^{*}\right)\right) \\
& F\left(x, x^{*} ; y, y^{*}\right)=\left\{x \alpha+x^{*} \gamma, y \beta\right\}+\left\{y^{*} \gamma, x \beta+x^{*} \delta\right\}^{\iota}
\end{aligned}
$$

du fait que $\sigma \sigma^{\mathrm{I}}=1, F$ est ici une forme hermitienne sur $X \oplus X^{*}$. Il s'ensuit que $P s(X / \mathcal{A})$ est isomorphe à $S p(X / \mathcal{A})$. De plus, d'après le $n^{\circ} 31$ de [14], la condition de symplecticité de $\sigma$ équivaut à dire que $\sigma$ laisse invariante la forme $k$-bilinéaire alternée

$$
\left[x_{1}, x_{2}^{*}\right]-\left[x_{2}, x_{1}^{*}\right]=\tau\left(\left\{x_{1}, x_{2}^{*}\right\}-\left\{x_{2}, x_{1}^{*}\right\}^{\prime}\right)
$$

En remplaçant $\left(x_{1}, x_{1}^{*}\right)$ par $\left(t x_{1}, t x_{1}^{*}\right)$ avec $t \in \mathcal{A}$, on voit que cela revient à dire que $\sigma$ laisse invariante la forme $\left\{x_{1}, x_{2}^{*}\right\}-\left\{x_{2}, x_{1}^{*}\right\}^{\imath}$, qui est sesquilinéaire et antihermitienne sur $\left(X \oplus X^{*}\right) \times\left(X \oplus X^{*}\right)$.

15. Comme il est bien connu, on peut former le produit tensoriel sur $\mathcal{A}$ d'un $\mathcal{A}$-module à droite $X$ et d'un $\mathcal{A}$-module à gauche $Y$; c'est l'espace vectoriel sur $k$, quotient du produit tensoriel $X \otimes_{k} Y$ de $X$ et $Y$ (ou plutôt des espaces vectoriels sur $k$, respectivement sousjacents à $X$ et à $Y$ ), pris sur $k$, par le sous-espace de ce produit engendré par les élements $x t \otimes y-x \otimes t y$ pour $x \in X, y \in Y, t \in \mathcal{A}$. Mais, au moyen de l'involution $\iota$, tout $\mathcal{A}$-module à gauche $X$ peut être considéré aussi comme $\mathcal{A}$-module à droite, par la formule $x t=t^{t} x(x \in X, t \in \mathcal{A})$. Cela conduit à définir un produit tensoriel, qu'on notera $\otimes_{t}$, entre $A$-modules à gauche $X, Y$; par définition, $X \otimes_{\iota} Y$ sera l'espace vectoriel sur $k$, quotient de $X \otimes_{k} Y$ par le sous-espace engendré par les éléments $t^{t} x \otimes y-x \otimes t y(x \in X, y \in Y, t \in \mathcal{A})$; l'image de $x \otimes y$ dans ce quotient sera notée $x \otimes_{s} y$.

Comme le dual de $X \otimes_{k} Y$ n'est autre que l'espace des formes $k$-bilinéaires sur $X \times Y$, le dual de $X \otimes_{\imath} Y$ s'identifie à l'espace des formes $k$-bilinéaires $f$ sur $X \times Y$ qui satisfont à $f\left(t^{t} x, y\right)=f(x, t y)$ quels que soient $x \in X, y \in Y, t \in \mathcal{A}$. Mais, si $x \in X, y \in Y$, l'application $t \rightarrow f(t x, y)$ est une forme $k$-linéaire sur $\mathcal{A}$, qu'on peut donc, d'une manière et d'une seule, écrire sous la forme $t \rightarrow \tau(t F(x, y))$, où $F$ est une application de $X \times Y$ dans $\mathcal{A}$; on vérifie alors immédiatement que, dans ces conditions, $F$ est sesquilinéaire. Réciproquement, ces formules font correspondre à toute forme sesquilinéaire sur $X \times Y$ un élément du dual de $X \otimes_{\imath} Y$. Il revient au même de dire que ce dual s'identifie à l'espace des formes sesquilinéaires $F$ sur $X \times Y$ au moyen de la formule

$$
[x \otimes, y, F]=\tau(F(x, y))
$$

Nous aurons à peu près exclusivement à faire usage de ces notions dans le cas où $X=Y$. Dans ce cas, soit $s$ l'automorphisme d'ordre 2 de $X \otimes_{\imath} X$ défini par $s\left(x \otimes_{\imath} y\right)=y \otimes_{\imath} x$. Comme la caractéristique de $k$ n'est pas $2, X \otimes_{\imath} X$ est somme directe de l'espace $I(X)$ 
des éléments invariants par $s$ et de l'espace $I^{-}(X)$ des éléments $u$ tels que $s u=-u ; I(X)$ et $I^{-}(X)$ sont les sous-espaces de $X \otimes_{\imath} X$ respectivement engendrés par les éléments de la forme $x \otimes_{\iota} x$ (pour $x \in X$ ) et par les éléments de la forme $x \otimes_{\iota} y-y \otimes_{\imath} x$ (pour $x \in X, y \in X$ ). De plus, on peut, d'une manière évidente, identifier $I(X)$ avec $\left(X \otimes_{t} X\right) / I^{-}(X)$.

Une fois pour toutes, on conviendra de noter $i_{X}$ l'application $x \rightarrow x \otimes_{\imath} x$ de $X$ dans $I(X)$, c'est-à-dire qu'on posera $i_{X}(x)=x \otimes_{\imath} x$. Cette application est évidemment quadratique, c'est-à-dire que, pour un choix quelconque de bases de $X$ et de $I(X)$ sur $k$, les coordonnées de $i_{X}(x)$ s'expriment comme polynomes homogènes du second degré par rapport à celles de $x$.

Soit alors $F$ une forme sesquilinéaire sur $X \times X$, que nous identifions à un élément du dual de $X \otimes_{\imath} X$ au moyen de (15). Si $F$ est hermitienne, on aura, quels que soient $x, y$ :

$$
\left[x \otimes, y, F^{\prime}\right]=\left[y \otimes, x, F^{\prime}\right]
$$

done $[u, F]=0$ pour $u \in I^{-}(X)$; autrement dit, $F$ appartient au dual de $\left(X \otimes_{\imath} X\right) / I^{-}(X)$, c'est-à-dire, d'après les conventions ci-dessus, au dual $I(X)^{*}$ de $I(X)$, considéré comme sous-espace du dual de $X \otimes_{\imath} X$. Réciproquement, s'il en est ainsi, on aura, quels que soient $x \in X, y \in X, t \in \mathcal{A}$ :

$$
\tau(F(t x, y))=\tau(F(y, t x)),
$$

ce qui s'écrit aussi, puisque $F$ est sesquilinéaire et que $\tau$ est invariante par $\iota$ :

$$
\tau(t F(x, y))=\tau\left(F(y, x) t^{t}\right)=\tau\left(t \cdot F(y, x)^{x}\right)
$$

cette relation ayant lieu quel que soit $t$, il s'ensuit que $F$ est hermitienne. Pour $x=y$, les formules ci-dessus donnent

$$
\left[i_{X}(x), F\right]=\tau(F(x, x)),
$$

de sorte qu'on peut identifier $I(X)^{*}$ avec l'espace des formes hermitiennes sur $X \times X$ au moyen de cette formule. On a vu d'ailleurs au $n^{\circ} 49$ de [14] que ce dernier espace s'identifie avec l'espace $Q(X / \mathcal{A})$ des formes $\mathcal{A}$-quadratiques $f$ sur $X$ au moyen de $f(x)=\tau(F(x, x)$ ); il s'ensuit que la formule $f(x)=\left[i_{X}(x), f\right]$ permet d'identifier $Q(X / \mathcal{A})$ avec $I(X)^{*}$.

Soit encore $Z$ un sous-module de $X$, et supposons que $Z$ admette un supplémentaire $Z^{\prime}$ dans $X$ (ce qui sera toujours le cas si $\mathcal{A}$ est semisimple); on peut donc écrire $X=Z \oplus Z^{\prime}$. Il est immédiat qu'alors $I(X)$ s'identifie canoniquement à la somme directe de $I(Z), Z \otimes \otimes_{1} Z^{\prime}$ et $I\left(Z^{\prime}\right)$, et que le sous-espace de $I(X)$ engendré par les éléments $i_{X}(z)$ pour $z \in Z$ est $I(Z)$. Autrement dit, on a le droit, dans ces conditions, d'identifier $I(Z)$ avec le sous-espace de $I(X)$ engendré par l'image de $Z$ par $i_{X}$, et d'identifier $i_{Z}$ avec $i_{X}$. 
On notera aussi que, si $K$ est un corps contenant $k, I\left(X_{K}\right)$ s'identifie à $I(X)_{K}$, et par suite $Q\left(X_{K} / \mathcal{A}_{K}\right)$ à $Q(X / \mathcal{A})_{K}$. On notera toujours $i_{X}$ l'application de $X_{K}$ dans $I(X)_{K}$ déduite de la manière évidente de $i_{X}$.

La propriéte suivante de $i_{X}$ jouera un rôle important par la suite :

Lемм т. Pour que l'application $i_{X}$ de $X$ dans $I(X)$ soit submersive en un point $x$ de $X$, il faut et il suffit qu'on ait $I(X / \mathcal{A} x)=\{0\}$, ou en d'autres termes que l'espace $Q((X / \mathcal{A} x) / \mathcal{A})$ soit réduit à $\{0\}$.

La différentielle de $i_{X}$ en $x$ est en effet donnée par

$$
d i_{X}(x)=x \otimes_{\imath} d x+d x \otimes_{\imath} x
$$

de sorte que l'application linéaire de $X$ dans $I(X)$, tangente à $i_{X}$ en $x$, est $y \rightarrow x \otimes_{\imath} y+y \otimes_{\imath} x$. Pour que celle-ci ne soit pas surjective, il faut et il suffit qu'il y ait $f \neq 0$ dans $I(X)^{*}$ telle que l'on ait

$$
\left[x \otimes, y+y \otimes_{\imath} x, f\right]=0
$$

quel que soit $y$. Identifiant $I(X)^{*}$ avec l'espace des formes hermitiennes sur $X \times X$ comme il a été dit plus haut, on voit que cette condition équivaut à la suivante : il existe sur $X \times X$ une forme hermitienne $F \neq 0$ telle que $\tau(F(x, y))=0$ pour tout $y \in X$. En remplaçant $y$ par ty, donc $F(x, y)$ par $F(x, y) t^{\imath}$, dans cette condition, on voit qu'elle revient à dire que $F(x, y)=0$ quel que soit $y$; en remplaçant alors $x$ par $t x$, on voit que cela revient à dire que $F(z, y)=0$ quels que soient' $z \in A x$ et $y \in X$. Posons $Z=A(x ; Z$ est le sous-module de $X$ engendré par $x$. Par passage au quotient, l'espace des formes hermitiennes sur $X \times X$ ayant la propriété qu'on vient d'énoncer s'identifie à l'espace des formes hermitiennes sur $(X / Z) \times(X / Z)$, c'est-à-dire au dual de $I(X / Z)$, ou encore à $Q((X / Z) / \mathcal{A})$, ce qui achève la démonstration.

16. Désormais, nous supposerons une fois pour toutes que l'algèbre $\mathcal{A}$ est semisimple. Nous allons d'abord énoncer, dans le langage qui nous sera commode, quelques-uns des théorèmes de structure classiques au sujet de ces algèbres, et fixer quelques notations.

Une telle algèbre $\mathcal{A}$, comme on sait. est somme directe d'une famille finie d'algèbres simples $\mathcal{A}_{\nu}(\nu \in N)$; pour chaque $\nu$, on choisira une fois pour toutes un $\mathcal{A}_{\nu}$-module à gauche simple $A_{v}$, qu'on pourra aussi considérer d'une manière évidente comme $\mathcal{A}$-module (l'annulateur de $A_{\nu}$ dans $\mathcal{A}$ étant la somme des $\mathcal{A}_{\mu}$ pour $\left.\mu \neq \nu\right)$. On notera $A$ la somme directe des $A_{v}$, considérée comme $\mathcal{A}$-module à gauche. L'anneau $\operatorname{End}\left(A_{v}\right)$ des endomorphismes de $A_{v}$ considéré comme module à gauche, soit sur $\mathcal{A}_{v}$, soit sur $\mathcal{A}$, est une algèbre à division 
sur $k$ (donc un corps, commutatif ou non) qu'on notera $\mathfrak{l}_{v} ; A_{v}$ est alors un espace vectoriel à droite sur $\mathfrak{l}_{v}$, dont la dimension sera notée $m_{v}$, et $\mathcal{A}_{v}$ n'est pas autre chose que l'anneau des endomorphismes de cet espace vectoriel. Par suite, $\mathcal{A}_{v}$ est isomorphe à l'anneau $M_{m_{\nu}}\left(\mathfrak{f}_{v}\right)$ des matrices d'ordre $m_{v}$ sur $\mathfrak{k}_{\nu}$. On désignera par $\boldsymbol{B}$ la somme directe des $\mathfrak{l}_{\nu}$; alors les $A_{v}$, et par suite $A$, peuvent, d'une manière évidente, être considérés comme $B$-modules à droite; $B$, opérant ainsi sur $A$, n'est autre d'ailleurs que l'anneau $\operatorname{End}_{A}(A)$ des endomorphismes de $A$ considéré comme $\mathcal{A}$-module à gauche. Réciproquement, $\mathcal{A}$, opérant à gauche sur $A$, est l'anneau $\operatorname{End}_{B}(A)$ des endomorphismes de $A$ considéré comme $\mathcal{B}$-module à droite. Comme les opérations de $\mathcal{A}$ et de $B$ sur $A$ sont permutables les unes avec les autres, on peut considérer $A$ comme bimodule sur $\mathcal{A}$ et sur $\boldsymbol{B}$.

Soit $X$ un $A$-module à gauche; il est somme directe de modules simples, dont chacun est isomorphe à l'un des $A_{v}$; on désignera par $X_{v}$ la somme de celles des composantes simples de $X$ qui sont isomorphes à $A_{\nu}$, et par $n_{v}$ le nombre de ces composantes. La famille d'entiers $n=\left(n_{v}\right)_{v \in N}$ s'appellera le rang de $X ; X$ est déterminé d'une manière unique par son rang, ̀̀ un isomorphisme près. Si $x \in X$, le rang du sous-module $\mathcal{A} x$ de $X$ engendré par $x$ s'appellera le rang de $x$; si celui-ci est égal au rang de $X$, c'est-à-dire si $x$ engendre $X$, on conviendra de dire que $x$ est de rang maximal dans $X$; pour qu'il existe de tels éléments dans $X$, il faut et il suffit que $X$ soit " monogène ».

17. Avec les mêmes notations que ci-dessus, soit encore $B=\operatorname{Hom}_{A}(X, A)$; c'est l'ensemble des applications $\mathcal{A}$-linéaires de $X$ dans $A$, ceux-ci étant considérés comme $\mathcal{A}$ modules à gauche; on considérera $B$ comme opérant à droite sur $X$, et on lui donnera sa structure "naturelle " de $\mathcal{B}$-module à droite. De même, soit $B_{v}$ l'ensemble des applications $A_{v}$-linéaires de $X_{v}$ dans $A_{v} ; B_{v}$ est « naturellement " espace vectoriel à droite sur $\mathfrak{l}_{v}$, et, comme tel, il a la dimension $n_{v} ; B$ est alors somme directe des $B_{v}$. L'application de $X$ dans $A$ définie par un élément $b$ de $B$ étant notée $x \rightarrow x b$, il s'ensuit que, pour tout $x \in X, b \rightarrow x b$ est une application $\mathcal{B}$-linéaire de $B$ dans $A$, ceux-ci étant considérés comme $\mathcal{B}$-modules à droite; et toute application $\mathcal{B}$-linéaire de $B$ dans $A$ est de cette forme. Autrement dit, on peut identifier $X$ avec l'espace $\operatorname{Hom}_{\mathfrak{B}}(B, A)$ de ces applications. Cette identification permet d'énoncer le lemme suivant :

Lем т 8. Soient $\mathcal{A}, A, X, B$ comme ci-dessus; soient $x_{0}, x_{1}$ deux éléments de $X$, et soient $N_{0}, N_{1}$ leurs noyaux quand on les considère comme applications de $B$ dans $A$. Alors, pour que $\mathcal{A} x_{0} \supset \mathcal{A} x_{1}$, il faut et il suffit que $N_{0} \subset N_{1}$; pour que $\mathcal{A} x_{0}=X$, il faut et il suffit que $N_{0}=\{0\}$.

Il est clair que $\mathcal{A} x_{0} \supset \mathcal{A} x_{1}$ équivaut à $x_{1} \in \mathcal{A} x_{0}$ et entraîne $N_{0} \subset N_{1}$. Pour démontrer a réciproque, on peut se borner au cas où $\mathcal{A}$ est une algèbre simple, le cas général résultant 
trivialement de celui-ci. On peut done supposer que $A$ et $B$ sont des espaces vectoriels à droite sur un corps $\mathfrak{l}$ et que $A=\operatorname{End}_{\mathfrak{f}}(A)$ et $X=\operatorname{Hom}_{\mathfrak{f}}(B, A)$. Soient $C_{0}, C_{1}$ les images de $B$ dans $A$ par $x_{0}, x_{1}$; par passage au quotient, $x_{0}$ détermine un isomorphisme $u_{0}$ de $B / N_{0}$ sur $C_{0}$, et, si $N_{1} \supset N_{0}, x_{1}$ détermine une application f-linéaire $u_{1}$ de $B / N_{0}$ sur $C_{1}$. Il existe alors un endomorphisme $a$ de $A$ qui coïncide avec $u_{1} \circ u_{0}^{-1}$ sur $C_{0}$; par exemple, on peut achever de le déterminer en lui imposant de s'annuler sur un supplémentaire arbitrairement choisi de $C_{0}$ dans $A$. On a alors $x_{1}=a x_{0}$, ce qui démontre la première partie du lemme. La seconde s'ensuit si on observe qu'à tout $b \in B$ correspond au moins un $x \in X$ tel que $x b \neq 0$.

Il résulte en particulier du lemme 8 que, si $\mathcal{A}$ est une algèbre simple, le rang d'un élément $x$ de $X$, tel qu'il a été défini ci-dessus, n'est autre que le rang de $x$ en tant qu'application $\mathfrak{k}$-linéaire de $B$ dans $A$.

18. Si on s'est donné de plus une involution $\iota$ sur $\mathcal{A}$, celle-ci détermine sur l'ensemble $N$ des composantes simples $\mathcal{A}_{\nu}$ de $\mathcal{A}$ une permutation $\boldsymbol{v} \rightarrow \boldsymbol{v}^{\imath}$ d'ordre 2. La détermination de $A$, en tant qu'algèbre à involution, se ramène alors à celle des " algèbres à involution simples » $\mathcal{A}_{\nu}$ (pour $\nu=\nu^{\imath}$ ) et $\mathcal{A}_{v} \oplus A_{\mu}$ (pour $v^{\imath}=\mu \neq v$ ). Comme on sait (cf. p. ex. [1], Chap. X), celles-ci sont nécessairement, à un isomorphisme près, de l'un des types suivants :

(I) $\nu=v^{\imath} ; \mathcal{A}_{\nu}$ est l'algèbre de matrices $M_{m}(\mathfrak{f})$ sur une algèbre à division $\mathfrak{f}$ munie d'une involution $\xi \rightarrow \xi^{\prime}$, et $\iota$ est l'involution $x \rightarrow h^{-1} \cdot x^{\prime} \cdot h$, où $h$ est une matrice inversible satisfaisant à ${ }^{t} h^{\prime}=\eta h$, avec $\eta= \pm 1$;

(II) $v^{\imath}=\mu \neq v ; \mathcal{A}_{\nu}$ est l'algèbre de matrices $M_{m}(\mathfrak{f})$ sur une algèbre à division $\mathfrak{f} ; \mathcal{A}_{\mu}$ est l'algèbre $M_{m}\left(\mathfrak{f}^{\prime}\right)$, où $\mathfrak{f}^{\prime}$ est telle qu'il existe un anti-isomorphisme $\alpha$ de $\mathfrak{l}$ sur $\mathfrak{f}^{\prime}$; et $\iota$ est l 'involution $(x, y) \rightarrow\left({ }^{t} y^{\alpha^{-1}},{ }^{t} x^{\alpha}\right)$ de $M_{m}(\mathfrak{f}) \oplus M_{m}\left(\mathfrak{l}^{\prime}\right)$.

Pour des raisons évidentes, la plupart des problèmes qu'on peut se poser au sujet des algèbres semisimples à involution se ramènent aux problèmes correspondants dans les cas " simples " (I) et (II). Nous allons maintenant achever de fixer les notations en ce qui concerne ceux-ci et démontrer divers résultats auxiliaires.

Dans le cas $(\mathrm{I})$, on se donne donc une algèbre à division $\mathfrak{l}_{\text {sur }} k$, munie d'une involution $\xi \rightarrow \xi^{\prime}$. On notera $z$ le centre de $\mathfrak{k}$, et $z_{0}$ le sous-corps de $z$ formé des éléments de $z$ invariants par l'automorphisme induit sur $z$ par $\xi \rightarrow \xi^{\prime}$; suivant que ce dernier est ou non l'automorphisme identique, on $a z=z_{0}$, ou bien $z$ est une extension quadratique de $z_{0}$. Si $V$ est un espace vectoriel sur $k$, on étendra $\xi \rightarrow \xi^{\prime}$ à une application $k$-linéaire de $V \otimes_{k} \mathfrak{k}$ sur lui-même en posant $(v \otimes \xi)^{\prime}=v \otimes \xi^{\prime}$ pour $v \in V, \xi \in \mathfrak{l}$. En particulier, si $V$ est l'espace $M_{m, n}(k)$ des matrices à $m$ lignes et $n$ colonnes sur $k, V \bigotimes_{k} \mathfrak{f}$ sera l'espace analogue $M_{m, n}(\mathfrak{f})$ sur $\mathfrak{l}$, et, si $x \in M_{m, n}(\mathfrak{f}), x^{\prime}$ sera la matrice obtenue en appliquant à chaque élément de la 
matrice $x$ l'involution $\xi \rightarrow \xi^{\prime}$. On écrira $M_{m}(k), M_{m}(\mathfrak{f})$ au lieu de $M_{m, m}(k), M_{m, m}(\mathfrak{f})$. Pour tout $m, u \rightarrow^{t} u^{\prime}$ est une involution de $M_{m}(\mathfrak{f})$. On notera $\tau_{m}$ la trace réduite prise dans $M_{m}(\mathfrak{f})$ sur zo considéré comme corps de base.

Soit $h$ un élément inversible de $M_{m}(\mathfrak{l})$ tel que ${ }^{t} h^{\prime}=\eta h$ avec $\eta= \pm 1$. On prend alors pour $\mathcal{A}$ l'algèbre $M_{m}(\mathfrak{f})$ munie de l'involution $\iota$ définie par $u^{\iota}=h^{-1} \cdot{ }^{t} \cdot h$. On vérifie facilement que, si $\lambda$ est une forme $k$-linéaire sur $z_{0}, \tau=\lambda \circ \tau_{m}$ est une fonction trace sur $\mathcal{A}$ pourvu que $\lambda \neq 0$, et que réciproquement toute fonction trace sur $\mathcal{A}$ est de cette forme.

Conformément aux conventions du $\mathrm{n}^{\circ} 16$, on posera ici $A=M_{m .1}(\mathfrak{f})$; $\mathcal{A}$ opérant sur $A$ par multiplication matricielle, $A$ est bien un $A$-module simple à gauche. De même, pour tout $n, M_{m, n}(\mathfrak{t})$ est, pour $\mathcal{A}$ opérant à gauche par multiplication matricielle, un $\mathcal{A}$ module de rang $n$. Quels que soient $n$ et $p$, toute application $\mathcal{A}$-linéaire de $M_{m, n}(\mathfrak{f})$ dans $M_{m, p}(\mathfrak{f})$ est de la forme $x \rightarrow x \alpha$ avec $\alpha \in M_{n, p}(\mathfrak{f})$. En particulier, si on pose $X=M_{m, n}(\mathfrak{f})$ et, comme au $\mathrm{n}^{\circ} 17, B=\operatorname{Hom}_{A}(X, A)$, on peut identifier $B$ avec $M_{n, 1}(\mathfrak{f})$, puis $X$ avec $\operatorname{Hom}_{\mathfrak{k}}(B, A)$, c'est-à-dire avec l'espace des applications $\mathfrak{k}$-linéaires de $B$ dans $A$ lorsque ceux-ci sont considérés comme espaces vectoriels à droite sur $\mathfrak{l}$. Comme on l'a déjà observé, le rang d'un élément $x$ de $X$ au sens du $n^{\circ} 16$ coïncide avec son rang en tant que matrice à $m$ lignes et $n$ colonnes sur $\mathfrak{1}$. On notera que l'anneau $\operatorname{End}_{A}(X)$ des endomorphismes de $X$ considéré comme $A$-module à gauche, et l'anneau $\operatorname{End}_{\mathfrak{f}}(B)$ des endomorphismes de $B$ considéré comme espace vectoriel à droite sur $\mathfrak{f}$, s'identifient tous deux à $M_{n}(\mathfrak{f})$ et s'identifient donc l'un avec l'autre.

19. Si $X=M_{m, n}(\mathfrak{l}), Y=M_{m, p}(\mathfrak{t})$, on voit aisément que toute forme sesquilinéaire $F$ sur $X \times Y$ peut s'écrire $F(x, y)=x \cdot w^{\cdot t}(h y)^{\prime}$, avec $w \in M_{n, p}(\mathfrak{t})$; l'espace de ces formes, c'està-dire, d'après le $\mathrm{n}^{\circ} 15$, le dual de $X \otimes_{\imath} Y$, peut donc alors s'identifier avec $M_{n, p}(\mathfrak{l})$ au moyen de cette formule; si l'on fait cette identification, (15) s'écrit

$$
[x \otimes, y, w]=\tau\left(x w \cdot{ }^{t}(h y)^{\prime}\right)=\lambda\left(\tau_{n}\left(w \cdot{ }^{t}(h y)^{\prime} \cdot x\right)\right) .
$$

Mais $\left(w_{1}, w_{2}\right) \rightarrow \lambda\left(\tau_{n}\left(w_{1} \cdot{ }^{t} w_{2}^{\prime}\right)\right)$ est une forme bilinéaire non dégénérée sur $M_{n, p}(\mathfrak{f}) \times M_{n, p}(\mathfrak{f})$; si on identifie $M_{n, p}(\mathfrak{k})$ avec son dual au moyen de cette forme, on voit que $X \otimes, Y$ s'identifie aussi avec $M_{n, p}(\mathfrak{f})$, au moyen de la formule $x \otimes_{\imath} y={ }^{t} x^{\prime} \cdot h \cdot y$. En particulier, pour $X=Y$, c'est-à-dire pour $n=p$, on voit que l'automorphisme $s$ de $X \otimes_{\imath} X$ défini par $s\left(x \otimes_{\imath} y\right)=y \otimes_{\imath} x$ devient l'automorphisme $w \rightarrow \eta^{t} w^{\prime}$ de $M_{n}(\mathfrak{f}) ; I(X)$ est l'espace des $w \in M_{n}(\mathfrak{l})$ tels que $w=\eta^{t} w^{\prime}$, et $i_{X}$ est l'application $x \rightarrow^{t} x^{\prime} \cdot h \cdot x$ de $X$ dans $I(X)$.

Suivant l'usage, si $V$ et $W$ sont des espaces vectoriels à droite sur $\mathfrak{k}, \varphi$ une application f-linéaire de $V$ dans $W$, et $f$ une forme sesquilinéaire (par exemple $\eta$-hermitienne) sur $W \times W$, on notera $f[\varphi]$ la forme sesquilinéaire $f \circ(\varphi, \varphi)$ sur $V \times V$, où $(\varphi, \varphi)$ désigne l'applica- 
tion $\left(v_{1}, v_{2}\right) \rightarrow\left(\varphi\left(v_{1}\right), \varphi\left(v_{2}\right)\right)$ de $V \times V$ dans $W \times W$. Les espaces $A, X, B$ étant comme ci-dessus, considérons alors sur $A \times A$ la forme $\eta$-hermitienne définie par $\left(a_{1}, a_{2}\right)^{\rightarrow}{ }^{t} a_{1}^{\prime} \cdot h \cdot a_{2}$, et convenons de la désigner aussi par $h$; de même, à tout $w \in M_{n}(\mathfrak{f})$ tel que $w=\eta^{t} w^{\prime}$, faisons correspondre la forme $\eta$-hermitienne $\left(b_{1}, b_{2}\right) \rightarrow^{t} b_{1}^{\prime} \cdot w \cdot b_{2}$ sur $B \times B$, et identifions $I(X)$ avec l'espace des formes $\eta$-hermitiennes sur $B \times B$ au moyen de cette correspondance. Il résulte alors de ce qui précède que, si on identifie $X$ avec $\operatorname{Hom}_{\mathfrak{f}}(B, A)$ comme plus haut, $i_{X}$ n'est autre que l'application $x \rightarrow h[x]$ de ce dernier espace dans l'espace des formes $\eta$-hermitiennes sur $B \times B$.

Lемме 9. Soient respectivement $\delta$ et $\delta^{\prime}$ les dimensions, sur $k$, de $\mathrm{f}$ et de l'espace des éléments $\xi$ de $\mathfrak{l}$ tels que $\xi^{\prime}=\eta \xi$, et soit $\varepsilon=\delta^{\prime} / \delta$. Alors, si $X$ est un A-module de rang $n, I(X)$ a la dimension $\delta n(n+2 \varepsilon-1) / 2$ sur $k$. De plus, pour que $i_{X}$ soit submersive en un point $x$ de $X$, il faut et il suffit que $x$ soit de rang $n$ ou bien que $\varepsilon=0$ et que $x$ soit de rang $n-1$.

La première assertion résulte de ce qui précède; la seconde suit de là, du lemme 7 du $n^{\circ} 15$, et du fait que, si $x$ est de rang $r, X / A x$ est de rang $n-r$.

20. Nous plaçant toujours dans le cas (I), nous allons, avec les notations ci-dessus, préciser la structure du groupe $P s(X / \mathcal{A})$ quand $X$ est un $\mathcal{A}$-module de rang $n$.

Soit $\varphi$ un isomorphisme de $X$ sur $M_{m . n}\left(\ell^{\natural}\right)$. Comme $X^{*}$ a même dimension que $X$ sur $k$, à savoir $\delta m n, X^{*}$ est aussi de rang $n$. Au moyen des résultats du $\mathbf{n}^{\circ} 19$, on voit immédiatement alors qu'on peut choisir, d'une manière et d'une seule, un isomorphisme $\psi$ de $X^{*} \operatorname{sur} M_{m, n}(\mathfrak{f})$ de manière à avoir

$$
\left\{x, x^{*}\right\}=(x \varphi) \cdot{ }^{t}\left(x^{*} \psi\right)^{\prime} \cdot h
$$

Désignons par $\omega$ l'isomorphisme $\left(x, x^{*}\right) \rightarrow\left(x \varphi, x^{*} \psi\right)$ de $X \oplus X^{*}$ sur $M_{m, n}(\mathfrak{f}) \oplus \boldsymbol{M}_{m, n}(\mathfrak{f})$, ou, ce qui revient au même, sur $M_{m .2 n}(\mathfrak{l})$. D'après le $\mathrm{n}^{\circ} 14$, pour qu'un automorphisme $\sigma$ de $X \oplus X^{*}$ soit symplectique, il faut et il suffit qu'il laisse invariante la forme sesquilinéaire

$$
\left(\left(x_{1}, x_{1}^{*}\right),\left(x_{2}, x_{2}^{*}\right)\right) \rightarrow\left\{x_{1}, x_{2}^{*}\right\}-\left\{x_{2}, x_{1}^{*}\right\}^{\natural}
$$

sur $\left(X \oplus X^{*}\right) \times\left(X \oplus X^{*}\right)$. Comme le montre un calcul facile, il revient au même de dire que l'automorphisme $s=\omega \sigma \omega^{-1}$ de $M_{m, 2 n}(\mathfrak{l})$ laisse invariante la forme

$$
\left(u_{1}, u_{2}\right) \rightarrow u_{1} \cdot e \cdot t u_{2}^{\prime} \cdot h
$$

sur $M_{m, 2 n}(\mathfrak{f}) \times M_{m, 2 n}(\mathfrak{l})$, où $e$ désigne la matrice $(-\eta)$-hermitienne donnée par

$$
e=\left(\begin{array}{cc}
0 & 1_{n} \\
-\eta \cdot 1_{n} & 0
\end{array}\right)
$$


Mais cette condition équivaut évidemment à $s \cdot e \cdot{ }^{t} s^{\prime}=e$. Autrement dit, l'application $(\sigma, f) \rightarrow$ $\omega \sigma \omega^{-1}$ est un isomorphisme de $P s(X / \mathcal{A})$ sur le groupe des éléments $s$ de $M_{2 n}\left(\mathfrak{f}^{*}\right)$ qui satisfont à $s \cdot e \cdot{ }^{t} s^{\prime}=e$. De plus, il est clair que l'image de $P(X / \mathcal{A})$ par cet isomorphisme est l'ensemble des éléments de ce dernier groupe qui sont de la forme

avec $a, b, d$ appartenant à $M_{n}(\mathfrak{f})$.

$$
s=\left(\begin{array}{ll}
a & b \\
0 & d
\end{array}\right)
$$

21. Dans le cas (II), on procèdera de même en se donnant d'abord une algèbre à division $\mathfrak{t}$ sur $k$, dont le centre sera noté zo. On désignera par $\mathfrak{f}^{\prime}$ l'algèbre "opposée 》 à $k$, et par $\xi \rightarrow \xi^{\prime}$ l'antiautomorphisme "canonique " de $\mathfrak{f}$ sur $\mathfrak{l}^{\prime}$, ainsi que son inverse, de sorte qu'on a $\left(\xi^{\prime}\right)^{\prime}=\xi$ et que $\xi \rightarrow \xi^{\prime}$ induit l'identité sur zo (qui est donc le centre commun de $\mathfrak{f}$ et de $\left.\mathfrak{l}^{\prime}\right)$.

Pour simplifier les notations, on conviendra, dans ce $\mathrm{n}^{\mathrm{o}}$, d'écrire $M_{m, n}, M_{m, n}^{\prime}$ au lieu de $M_{m, n}(\mathfrak{f}), M_{m, n}\left(\mathfrak{f}^{\prime}\right)$, et de même $M_{m}, M_{m}^{\prime}$ au lieu de $M_{m}(\mathfrak{f}), M_{m}\left(\mathfrak{f}^{\prime}\right)$. On prendra $\mathcal{A}=M_{m} \oplus M_{m}^{\prime}$, l'involution $\iota$ sur $\mathcal{A}$ étant donnée par $(u, v)^{\imath}=\left({ }^{t} v^{\prime},{ }^{t} u^{\prime}\right)$. On notera $\tau_{m}$ la trace réduite prise, soit dans $M_{m}$, soit dans $M_{m}^{\prime}$, sur zo considéré comme corps de base. La fonction trace $\tau$ sur $\mathcal{A}$ sera alors donnée par

$$
\tau(u, v)=\lambda\left(\tau_{m}(u)+\tau_{m}(v)\right)
$$

où $\lambda$ est une forme $k$-linéaire sur zo, et $\lambda \neq 0$.

On posera $A=A_{0} \oplus A_{0}^{\prime}$, avec $A_{0}=M_{m, 1}$ et $A_{0}^{\prime}=M_{m, 1}^{\prime}$; on notera que $A_{0}^{\prime}$ peut s'identifier d'une manière évidente avec le dual de $A_{0}$ sur $\mathfrak{t}$. Tout $\mathcal{A}$-module de rang $(p, q)$ est isomorphe au module $M_{m, p} \oplus M_{m, a}^{\prime}, \mathcal{A}$ opérant sur celui-ci par multiplication matricielle dans chaque composante. Toute application $\mathcal{A}$-linéaire de $M_{m, p} \oplus M_{m, q}^{\prime}$ dans $M_{m, r} \oplus M_{m, s}^{\prime}$ est de la forme $(x, y) \rightarrow(x \alpha, y \beta)$, avec $\alpha \in M_{p . r}, \beta \in M_{q, s}^{\prime}$. En particulier, si on pose de nouveau $B=\operatorname{Hom}_{A}(X, A)$, et qu'on prenne $X=X_{0} \oplus X_{0}^{\prime}$ avec $X_{0}=M_{m, p}, X_{0}^{\prime}=M_{m, q}^{\prime}$, on aura $B=B_{0} \oplus B_{0}^{\prime}$ avec $B_{0}=$ $M_{p, 1}, B_{0}^{\prime}=M_{q, 1}^{\prime}$, puis $X_{0}=\operatorname{Hom}_{\mathfrak{f}}\left(B_{0}, A_{0}\right)$ et $X_{0}^{\prime}=\operatorname{Hom}_{\mathfrak{f}^{\prime}}\left(B_{0}^{\prime}, A_{0}^{\prime}\right)$.

Si $X$ est comme ci-dessus, et si $Z=M_{m, r} \oplus M_{m, s}^{\prime}$, toute forme sesquilinéaire sur $X \times Z$ s'écrit :

$$
((x, y),(z, t)) \rightarrow\left(x \cdot v \cdot{ }^{t} t^{\prime}, y \cdot w \cdot z^{\prime}\right)
$$

avec $v \in M_{p, s}$ et $w \in M_{q, r}^{\prime}$; cela permet d'identifier le dual de $X \otimes_{\imath} Y$ avec $M_{p, s} \oplus M_{q, r}^{\prime}$. Raisonnant comme au $\mathrm{n}^{\circ} 19$, on en conclut qu'on peut identifier $X \otimes_{\imath} X$ avec $M_{q, p} \oplus M_{p, q}^{\prime}$, puis qu'on peut identifier $I(X)$ avec $M_{a, p}$ de manière à avoir $i_{X}(x, y)={ }^{t} y^{\prime} \cdot x$. Cela donne en particulier : 
Lемме 10. Soit $\delta$ la dimension de $\mathfrak{1}$ sur $k$. Alors, si $X$ est un A-module de rang $(p, q)$, $I(X)$ a la dimension $\delta p q$ sur $k$; et, pour que $i_{X}$ soit submersive en un point $x$ de rang $\left(p^{\prime}, q^{\prime}\right)$ dans $X$, il faut et il suffit $q u^{\prime}$ on ait $p=p^{\prime}$ ou $q=q^{\prime}$.

Toujours avec les mêmes notations, on pourra identifier le dual $X^{*} \operatorname{de} X$ avec $M_{m, q} \oplus M_{m, p}^{\prime}$ de manière à avoir

$$
\left\{(x, y),\left(x^{*}, y^{*}\right)\right\}=\left(x \cdot{ }^{t}\left(y^{*}\right)^{\prime}, y \cdot{ }^{t}\left(x^{*}\right)^{\prime}\right)
$$

Le dual d'un module de rang $(p, q)$ est donc de rang $(q, p)$. En posant $n=p+q, X \oplus X^{*}$ s'identifie d'une manière évidente à $M_{m, n} \oplus M_{m, n}^{\prime}$; d'après ce qu'on a vu, l'anneau des endomorphismes de ce dernier module s'identifie à $M_{n} \oplus M_{n}^{\prime}$. Pour qu'un automorphisme $\sigma=(\lambda, \mu)$ de $X \oplus X^{*}$ soit symplectique, il faut et il suffit qu'il laisse invariante la forme sesquilinéaire

$$
\left\{\left(x_{1}, y_{1}\right),\left(x_{2}^{*}, y_{2}^{*}\right)\right\}-\left\{\left(x_{2}, y_{2}\right),\left(x_{1}^{*}, y_{1}^{*}\right)\right\}^{\ell}
$$

Un calcul facile montre que, si on pose

$$
e=\left(\begin{array}{rl}
0 & 1_{p} \\
-1_{q} & 0
\end{array}\right)
$$

cette condition équivaut à $\mu=e^{-1} \cdot{ }^{t} \lambda^{\prime}-1 \cdot e$. Il s'ensuit qu'avec ces notations l'application $(\sigma, f) \rightarrow \lambda$ est un isomorphisme de $\operatorname{Ps}(X / \mathcal{A})$ sur $G L(n, \mathfrak{f})$. On vérifie facilement alors que l'image de $P(X / \mathcal{A})$ par cet isomorphisme est le sous-groupe de $G L(n, \mathfrak{f})$ formé des éléments de la forme

$$
\lambda=\left(\begin{array}{ll}
\lambda_{1} & \lambda_{2} \\
0 & \lambda_{3}
\end{array}\right)
$$

avec $\lambda_{1} \in G L(p, \mathfrak{f}), \lambda_{2} \in M_{p, q}(\mathfrak{f}), \lambda_{3} \in G L(q, \mathfrak{t})$.

22. Revenant au cas général des $\mathrm{n}^{\circ \mathrm{s}}$ 16-17, nous désignerons par $G$ le groupe des éléments $u$ de $\mathcal{A}$ tels que $u \cdot u^{\imath}=1$. Il est clair que $G$ est le produit direct des groupes analogues relatifs aux composantes "simples" de $\mathcal{A}$, celles-ci pouvant être de type (I) ou (II). Dans le cas (I), avec les notations du $\mathrm{n}^{\circ} 18, G$ est le groupe des éléments $u$ de $M_{m}(\mathfrak{t})$ qui satisfont à̀ ${ }^{t} u^{\prime} \cdot h \cdot u=h$, c'est-à-dire à $h[u]=h$; autrement dit, si $\operatorname{Aut}(A)=G L(m, \mathfrak{f})$ désigne le groupe des automorphismes de l'espace vectoriel à droite $A=M_{m .1}(\mathfrak{f})$ sur $\mathfrak{f}, G$ est le sous-groupe $\operatorname{Aut}(A, h)$ de $\operatorname{Aut}(A)$ formé des éléments de $\operatorname{Aut}(A)$ qui laissent invariante la forme $\eta$-hermitienne $h$. Dans le cas (II), avec les notations du $\mathrm{n}^{\circ} 21, G$ est le groupe des éléments de $\mathcal{A}$ de la forme $\left(u,{ }^{t} u^{\prime-1}\right)$ avec $u \in \operatorname{Aut}\left(A_{0}\right)=G L(m, \mathfrak{t})$, et est donc isomorphe à ce dernier groupe. Dans le cas général, $G$ est donc un produit de "groupes classiques ".

La définition de $i_{X}$ montre qu'on a, quels que soient $x \in X, t \in \mathcal{A}$ :

3-652922. Acta mathematica. 113. Imprimé le 26 février 1965. 


$$
i_{X}(t x)=(t x) \otimes_{\iota}(t x)=x \otimes_{\iota} t^{t}(t x) .
$$

Il s'ensuit que, pour $u \in G$, on a $i_{X}(u x)=i_{X}(x)$ quel que soit $x \in X$. En d'autres termes, $i_{X}$ est invariant par $G$ opérant à gauche sur $X$.

Dans les chapitres suivants, on aura besoin de divers résultats sur les orbites de $G$, et de certains groupes apparentés à $G$, dans $X$; ces résultats vont être établis dans les $\mathrm{n}^{\text {os }}$ 22-25. Dans le cas où $\mathcal{A}$ est de type $(\mathrm{I})$, et où $X$ est de rang 1 sur $\mathcal{A}$, ils sont en substance contenus dans le théorème de Witt; le lecteur qui n'a que ce cas en vue (cf. l'Introduction) pourra donc se dispenser de lire la plus grande partie des démonstrations qui vont suivre.

Proposition 3. Pour tout $i \in I(X)$, soit $U(i)$ l'ensemble des éléments de $i_{X}^{-1}(\{i\}) q u i$ sont de rang maximal dans $X$; alors, si $U(i)$ n'est pas vide, c'est une orbite de $G$ dans $X$.

Soit $x_{0} \in U(i)$, ce qui veut dire qu'on a $i_{X}\left(x_{0}\right)=i$ et $\mathcal{A} x_{0}=X$. Il est clair que, si $x_{1}=u x_{0}$ avec $u \in G$, on a $x_{1} \in U(i)$. Pour démontrer la réciproque, il suffit évidemment de traiter les cas (I) et (II). Dans le cas (I), avec les notations des $n^{\text {os }} 18$-19, l'hypothèse signifie que $h\left[x_{0}\right]=h\left[x_{1}\right]=i$ et que $x_{0}, x_{1}$ sont de rang maximal, c'est-à-dire (d'après le lemme $8 \mathrm{du} \mathrm{n}^{\circ} 17$ ) qu'ils sont de noyau $\{0\}$ si on les considère comme applications de $B$ dans $A$. Alors $x_{0}, x_{1}$ déterminent des isomorphismes $\bar{x}_{0}, \bar{x}_{1}$ de $B$ sur des sous-espaces $L_{0}, L_{1}$ de $A$, et $z=\bar{x}_{1} \bar{x}_{0}^{-1}$ est un isomorphisme de $L_{0}$ sur $L_{1}$. Soient $h_{0}, h_{1}$ les formes $\eta$-hermitiennes induites par $h$ sur $L_{0} \times L_{0}$ et sur $L_{1} \times L_{1}$, respectivement. L'hypothèse $h\left[x_{0}\right]=h\left[x_{1}\right]$ équivaut à $h_{0}=h_{1}[z]$, c'est-à-dire que $z$, en un sens évident, est un isomorphisme de $\left(L_{0}, h_{0}\right)$ sur $\left(L_{1}, h_{1}\right)$. Le théorème de Witt dit alors que $z$ peut être prolongé à un automorphisme $u$ de $(A, h)$, c'est-àdire à un élément de $G$; et on a bien $x_{1}=u x_{0}$.

Dans le cas (II), reprenons les notations du $n^{\circ} 21$, et considérons deux éléments $\left(x_{0}, y_{0}\right)$, $\left(x_{1}, y_{1}\right)$ de $U(i)$. On voit, comme plus haut, que $y_{0}, y_{1}$ déterminent des isomorphismes $\bar{y}_{0}, \bar{y}_{1}$ de $B_{0}^{\prime}$ sur des sous-espaces $L_{0}^{\prime}, L_{1}^{\prime}$ de $A_{0}^{\prime}$, et $\bar{y}_{1} \bar{y}_{0}^{-1}$ est un isomorphisme de $L_{0}^{\prime} \operatorname{sur} L_{1}^{\prime}$, qu'on peut prolonger à un automorphisme $v$ de $A_{0}^{\prime}$, de sorte qu'on a $y_{1}=v y_{0}$. En remplaçant $\left(x_{0}, y_{0}\right) \operatorname{par}\left({ }^{t} v^{\prime}-1 \cdot x_{0}, v y_{0}\right)$, on est done ramené à traiter le cas où $y_{0}=y_{1}$. Soit alors $z_{0}={ }^{t} y_{0}^{\prime}$, de sorte qu'on a $z_{0} x_{0}=z_{0} x_{1}$. Comme $z_{0} \in M_{q, m}(\mathfrak{l})$, on peut considérer $z_{0}$ comme application ‡-linéaire de $A_{0}=M_{m .1}(\mathfrak{k})$ dans $M_{q .1}(\mathfrak{k})$; soit $N_{0}$ le noyau de cette application. D'autre part, $x_{0}, x_{1}$ déterminent des isomorphismes $\bar{x}_{0}, \bar{x}_{1}$ de $B_{0}$ sur des sous-espaces $L_{0}, L_{1}$ de $A_{0}$, de sorte que $\bar{u}=\bar{x}_{1} \bar{x}_{0}^{-1}$ est un isomorphisme de $L_{0}$ sur $L_{1}$. Comme on a $z_{0}\left(x_{1}-x_{0}\right)=0$, on a $\bar{u} a-a \in N_{0}$ quel que soit $a \in L_{0}$, et de même $\bar{u}^{-1} a-a \in N_{0}$ quel que soit $a \in L_{1}$; on en conclut aussitôt que $L_{0}+N_{0}=L_{1}+N_{0}$, et aussi que $\bar{u}$ induit sur $L_{0} \cap N_{0}$ un isomorphisme de $L_{0} \cap N_{0}$ sur $L_{1} \cap N_{0}$; on peut alors prolonger ce dernier isomorphisme à un automorphisme $v$ de $N_{0}$. Comme $\bar{u}$ et $v$ coĩncident sur $L_{0} \cap N_{0}$, il existe un endomorphisme $u_{0}$ de $L_{0}+N_{0}$ et un seul qui coïncide avec $\bar{u} \operatorname{sur} L_{0}$ et avec $v$ sur $N_{0}$; de même, il existe un endomorphisme $u_{1}$ de 
$L_{0}+N_{0}$ et un seul qui coïncide avec $\bar{u}^{-1} \operatorname{sur} L_{1}$ et avec $v^{-1}$ sur $N_{0}$. On a donc $u_{0} u_{1}=1$, de sorte que $u_{0}$ est un automorphisme de $L_{0}+N_{0}$. Soient enfin $L^{\prime}$ un supplémentaire de $L_{0}+N_{0}$ dans $A_{0}$, et $u$ l'automorphisme de $A_{0}$ qui coïncide avec $u_{0}$ sur $L_{0}+N_{0}$ et avec l'identité sur $L^{\prime}$. On a alors $x_{1}=u x_{0}$; et $u-1$ applique $A_{0}$ dans $N_{0}$, de sorte qu'on a $z_{0} u=z_{0}$, ou encore $y_{0}={ }^{t} u^{\prime-1} \cdot y_{0}$. Cela achève la démonstration de la proposition 3 .

Corollaire. Pour que deux points $x_{0}, x_{1}$ de $X$ appartiennent à une même orbite pour $G$, il faut et il suffit qu'on ait $i_{X}\left(x_{0}\right)=i_{X}\left(x_{1}\right)$ et $\mathcal{A} x_{0}=\mathcal{A} x_{1}$.

Ces conditions sont évidemment nécessaires. Supposons-les satisfaites, et soit $X^{\prime}=$ $\mathcal{A} x_{0}$. Comme $\mathcal{A}$ est semisimple, $X^{\prime}$ a un supplémentaire dans $X$; par suite, d'après le $\mathrm{n}^{\circ} 15$, on peut identifier $I\left(X^{\prime}\right)$ avec le sous-espace de $I(X)$ engendré par les éléments $i_{X}\left(x^{\prime}\right)$ pour $x^{\prime} \in X^{\prime}$, et $i_{X^{\prime}}$ avec l'application de $X^{\prime}$ dans ce sous-espace de $I(X)$ qui est induite par $i_{X}$. Alors $x_{0}$ et $x_{1}$ sont des éléments de $X^{\prime}$ de rang maximal dans $X^{\prime}$, et on a $i_{X^{\prime}}\left(x_{0}\right)=i_{X^{\prime}}\left(x_{1}\right)$; d'après la proposition 3 , ils appartiennent donc à une même orbite de $G$ dans $X^{\prime}$.

23. La proposition 3 montre que, si $U(i)$ n'est pas vide, c'est un espace homogène par rapport au groupe $G$. Pour déterminer complètement sa structure, il faut connaître le groupe de stabilité $g_{0}$, dans $G$, d'un élément $x_{0}$ de $U(i)$.

Ici encore, on peut se borner aux cas (I) et (II). Dans le cas (I), considérons $i$ comme forme $\eta$-hermitienne sur $B \times B$; soit $B^{\prime}$ le sous-espace de $B$ qui, par rapport à $i$, est orthogonal à tous les vecteurs de $B$, et soit $B^{\prime \prime}$ un supplémentaire de $B^{\prime}$ dans $B$; alors la forme $\eta$-hermitienne induite par $i$ sur $B^{\prime \prime} \times B^{\prime \prime}$ est non-dégénérée. Soit $r$ le rang de $i$; alors $B^{\prime}, B^{\prime \prime}$ sont de dimensions $n-r$ et $r$, respectivement. Soit $x_{0} \in U(i)$; comme $x_{0}$ est de rang maximal, il définit un isomorphisme $\bar{x}_{0}$ de $B$ sur un sous-espace $L_{0}$ de $A$; comme on a $i=h\left[x_{0}\right]$, la forme induite par $h$ sur $L_{0} \times L_{0}$ est celle qui se déduit de $i$ par $\bar{x}_{0}$. Par suite, si $L^{\prime}, L^{\prime \prime}$ sont les images de $B^{\prime}, B^{\prime \prime}$ par $x_{0}$, la forme induite par $h$ sur $L^{\prime \prime} \times L^{\prime \prime}$ est non-dégénérée, et $L^{\prime}$ est l'intersection de $L_{0}$ avec l'orthogonal de $L_{0}$ par rapport à $h$ dans $A$. Soit $M$ l'orthogonal de $L^{\prime \prime}$ par rapport à $h$ dans $A$; la forme induite par $h$ sur $M \times M$ est non-dégénérée; comme $L^{\prime}$ est un sous-espace "totalement isotrope " de $M$, on sait ([5], p. 21) qu'il existe un sousespace totalement isotrope $L_{1}^{\prime}$ de $M$, supplémentaire dans $M$ de l'orthogonal de $L^{\prime}$ dans $M$. Soit enfin $L_{1}$ l'orthogonal de $L^{\prime \prime}+L^{\prime}+L_{1}^{\prime}$ dans $A$. Il est facile de vérifier que $A$ est somme directe de $L^{\prime \prime}, L^{\prime}, L_{1}, L_{1}^{\prime}$; done $L_{1}$ est de dimension $m-2 n+r$, ce qui implique $m \geqslant 2 n-r$. De plus, la forme $h_{1}$ induite par $h$ sur $L_{1} \times L_{1}$ est non-dégénérée. Alors, si on prolonge tout élément de $\operatorname{Aut}\left(L_{1}, h_{1}\right)$ à un automorphisme de $A$ en lui imposant d'induire l'identité sur $L_{0}+L_{1}^{\prime}$, on obtient un sous-groupe $g_{1}$ du groupe de stabilité $g_{0}$ de $x_{0}$ dans $G$, qui est évidemment isomorphe à $\operatorname{Aut}\left(L_{1}, h_{1}\right)$; de plus, on voit facilement que $g_{0}$ est produit semidirect de $g_{1}$ et d'un groupe $g_{0}^{\prime}$ qui induit l'identité sur $L^{\prime \prime}$ et sur $L^{\prime}$, qui laisse invariants les espaces 
$L^{\prime}+L_{1}$ et $L^{\prime}+L_{1}+L_{1}^{\prime}=M$, et qui induit l'identité sur $\left(L^{\prime}+L_{1}\right) / L^{\prime}$ et sur $M /\left(L^{\prime}+L_{1}\right)$; g $g_{0}^{\prime}$ est donc unipotent, de sorte qu'en définitive $g_{0}$ est isomorphe à un produit semidirect de Aut $\left(L_{1}, h_{1}\right)$ et d'un groupe unipotent. En particulier, si $i$ est non-dégénéré, donc de rang $n$, $L^{\prime}$ et $L_{1}^{\prime}$ se réduisent à $\{0\}, L_{1}$ est de dimension $m-n$, et $g_{0}$ est isomorphe à $\operatorname{Aut}\left(L_{1}, h_{1}\right)$.

Dans le cas (II), soit $i \in M_{q . p}(\mathfrak{f})$, et soit $r$ le rang de $i ; U(i)$ est l'ensemble des couples $\left(x,{ }^{t} z^{\prime}\right)$ avec $x$ de rang $p$ dans $M_{m, p}(\mathfrak{f}), z$ de rang $q$ dans $M_{q, m}(\mathfrak{f})$, et $z x=i$. Soit $\left(x_{0},{ }^{t} z_{0}^{\prime}\right)$ un tel couple, et soit $g_{0}$ son groupe de stabilité dans $G$; quand on identifie $G$ avec $G L(m, \mathfrak{f})$ comme au $\mathrm{n}^{\circ} 22, g_{0}$ s'identifie au groupe des $u \in G L(m, \mathfrak{l})$ tels que $u x_{0}=x_{0}$ et $z_{0} u=z_{0}$. Soit $L_{0}$ l'image de $B_{0}=M_{p .1}(\mathfrak{f})$ par $x_{0}$ dans $A_{0}=M_{m .1}(\mathfrak{f}) ; L_{0}$ est de dimension $p$. Soit $N_{0}$ le noyau de $z_{0}$ considéré comme application de $A_{0}$ dans $M_{q .1}(\mathfrak{l}) ; N_{0}$ est de dimension $m-q$. Comme $i$ est de rang $r, z_{0}$ induit sur $L_{0}$ une application de rang $r$ de $L_{0}$ dans $M_{q, 1}(\mathfrak{f})$, de sorte que $L_{0} \cap N_{0}$ a la dimension $p-r$. Posons $L^{\prime}=L_{0} \cap N_{0}$; soient $L^{\prime \prime}, L_{1}$ des supplémentaires de $L^{\prime}$ dans $L_{0}$ et dans $N_{0}$, respectivement; $L_{1}$ est de dimension $m-p-q+r$, ce qui entraîne $m \geqslant p+q-r$. Soit encore $L_{1}^{\prime}$ un supplémentaire de $L_{0}+N_{0} \operatorname{dans} A_{0}$, et soit $M=L^{\prime}+L_{1}+L_{1}^{\prime}$. Si on prolonge tout automorphisme de $L_{1}$ à un automorphisme de $A_{0}$ en lui imposant d'induire l'identité sur $L_{0}+L_{1}^{\prime}$, on obtient un sous-groupe $g_{1}$ de $g_{0}$, isomorphe à Aut $\left(L_{1}\right)$. Soit $g_{0}^{\prime}$ le sous-groupe de $G$ qui induit l'identité sur $L^{\prime \prime}$ et sur $L^{\prime}$, qui laisse invariants les espaces $L^{\prime}+L_{1}$ et $M$, et qui induit l'identité sur $\left(L^{\prime}+L_{1}\right) / L^{\prime}$ et sur $M /\left(L^{\prime}+L_{1}\right)$. Alors $g_{0}$ est produit semidirect de $g_{1}$ et du groupe unipotent $g_{0}^{\prime}$. Si $p=q=r, L^{\prime}$ et $L_{1}^{\prime}$ se réduisent à $\{0\}, L_{1}$ est de dimension $m-p$, et $g_{0}$ est isomorphe à Aut $\left(L_{1}\right)$. Enfin, on notera que, dans le cas (II), la condition $m \geqslant p+q-r$ est, non seulement nécessaire, mais aussi suffisante pour que $U(i)$ ne soit pas vide.

24. En même temps que $G$, on fera opérer sur $X$, mais à droite, l'anneau $\operatorname{End}_{A}(X)$ des endomorphismes de $X$ considéré comme $\mathcal{A}$-module à gauche, et en particulier le groupe des éléments inversibles de cet anneau, c'est-à-dire des automorphismes de $X$, groupe qu'on notera simplement $\operatorname{Aut}(X)$. Si $B$ est défini comme au $n^{\circ} 17$, il est immédiat que $\operatorname{End}_{A}(X)$ s'identifie à l'anneau $\operatorname{End}_{B}(B)$ des endomorphismes de $B$ considéré comme $\mathcal{B}$ module à droite; par suite, $\operatorname{Aut}(X)$ s'identifie au groupe $\operatorname{Aut}(B)$ des automorphismes de $B$.

Par transport de structure, tout automorphisme $v$ de $X$ détermine un automorphisme de $I(X)$ qu'on notera $\bar{v}$, de sorte qu'on aura $i_{X}(x v)=i_{X}(x) \bar{v}$. Pour tout $i \in I(X)$, on notera $\operatorname{Aut}(X, i)$ le sous-groupe de $\operatorname{Aut}(X)$ qui laisse $i$ invariant, c'est-à-dire le groupe des $v \in \operatorname{Aut}(X)$ tels que $i \bar{v}=i$. On conviendra d'autre part de faire opérer le groupe $G \times \operatorname{Aut}(X)$ sur $X$ au moyen de la formule

$$
(u, v) \cdot x=u x v^{-1} \quad(u \in G, x \in X, v \in \operatorname{Aut}(X)) .
$$

Dans ces conditions, il est clair que l'ensemble $i_{X}^{-1}(\{i\})$ est invariant par le groupe 
$G \times \operatorname{Aut}(X, i)$. On va maintenant démontrer quelques résultats auxiliaires, qui serviront au Chapitre III, au sujet des orbites de ce dernier groupe et de certains de ses sous-groupes dans $i_{X}^{-1}(\{i\})$; dans le cas où $\mathcal{A}$ est de type $(\mathrm{I})$ et $X$ de rang 1 , ces résultats sont triviaux ou sont des conséquences triviales de ce qui précède.

Reprenant les notations du $n^{\circ} 17$, considérons d'abord l'espace $B^{*}=\operatorname{Hom}_{A}\left(X^{*}, A\right)$ attaché à $X^{*}$ comme $B$ l'est à $X$; supposant chaque composante "simple " de $\mathcal{A}$ mise sous la forme décrite au $\mathrm{n}^{\circ} 18$, et les composantes correspondantes de $X$ mises sous la forme décrite aux $n^{\text {os }} 19$ et 21 , nous allons définir un isomorphisme de $X \otimes_{6} X$ sur l'espace $\operatorname{Hom}_{\mathfrak{B}}\left(B, B^{*}\right)$ des morphismes de $B$ dans $B^{*}$ pour leurs structures de $B$-modules à droite; pour cela, il suffira évidemment de décrire cet isomorphisme séparément dans les cas (I) et (II); pour abréger, nous ne nous occuperons pas de savoir dans quel mesure il est "canonique n. Dans le cas (I), nous identifions $X$ avec $M_{m, n}(\mathfrak{f})$ et $X \otimes_{l} X$ avec $M_{n}(\mathfrak{f})$ comme au $\mathrm{n}^{\circ} 19$, cette dernière identification se faisant au moyen de la formule $x \otimes_{\imath} y={ }^{t} x^{\prime} \cdot h \cdot y$. Comme au $n^{\circ} 20$, identifions $X^{*}$ avec $M_{m, n}(\mathfrak{f})$ au moyen de la formule $\left\{x, x^{*}\right\}=x \cdot{ }^{t}\left(x^{*}\right)^{\prime} \cdot h$; puis identifions $B$ et $B^{*}$ avec $M_{n, 1}(\mathfrak{f})$ comme au $n^{\circ} 18$. Dans ces conditions, à tout élément $w$ de $X \otimes_{t} X$, on fera correspondre le morphisme $\lambda_{w}$ de $B$ dans $B^{*}$ donné par $\lambda_{w}(b)=w \cdot b$ pour tout $b \in B$. En particulier, pour $i \in I(X), \lambda_{i}$ sera donc le morphisme $b \rightarrow i \cdot b$; d'autre part, d'après le $\mathrm{n}^{\circ} 19, i$ satisfait alors à ${ }^{t} i^{\prime}=\eta \dot{ }$, et est la matrice de la forme $\eta$-hermitienne $\left(b_{1}, b_{2}\right) \rightarrow^{t} b_{1}^{\prime} \cdot i \cdot b_{2}$ sur $B \times B$; il s'ensuit que le noyau de $\lambda_{i}$ dans $B$ est le sous-espace $B^{\prime}$ de $B$ qui est orthogonal, par rapport à $i$, à tous les vecteurs de $B$. De plus, pour $x \in X$ et $i=i_{X}(x)$, on a $i={ }^{t} x^{\prime} \cdot h \cdot x$, de sorte que le noyau de $x$ dans $B$ est contenu dans celui de $\lambda_{i}$.

De même, dans le cas (II), on identifiera de nouveau $X$ avec $M_{m, p} \oplus M_{m, q}^{\prime}$ et $X \otimes_{\imath} X$ avec $M_{q, p} \oplus M_{p, q}^{\prime}$ comme au $\mathrm{n}^{\circ} 21$, cette dernière identification se faisant au moyen de la formule

$$
(x, y) \otimes_{\imath}(z, t)=\left({ }^{t} t^{\prime} \cdot x,{ }^{t} z^{\prime} \cdot y\right)
$$

pour $x$ et $z$ dans $M_{m, p}$ et $y$ et $t$ dans $M_{m, q}^{\prime}$. On identifiera $X^{*}$ avec $M_{m, q} \oplus M_{m, p}^{\prime}$ et $B$ avec $M_{p, 1} \oplus M_{q, 1}^{\prime}$ comme au $\mathrm{n}^{\circ} 21$, et de même $B^{*}$ avec $M_{q, 1} \oplus M_{p, 1}^{\prime}$ Alors, pour tout $(u, v) \in X \otimes_{t} X$, on définira le morphisme $\lambda_{(u, v)}$ de $B$ dans $B^{*}$ en posant, pour tout élément $(b, c)$ de $B$ :

$$
\lambda_{(u, v)}(b, c)=(u b, v c)
$$

En particulier, pour $(x, y) \in X$ et $i=i_{X}(x, y), \lambda_{i}$ sera donné, d'après le $n^{\circ} 21$, par la formule

$$
\lambda_{i}(b, c)=\left({ }^{t} y^{\prime} \cdot x \cdot b,{ }^{t} x^{\prime} \cdot y \cdot c\right)
$$

d'où il résulte que le noyau de $(x, y)$ dans $B$ est contenu dans celui de $\lambda_{i}$, comme dans le cas (I). 
25. Ces définitions étant étendues d'une manière évidente, composante par composante, au cas général où $\mathcal{A}$ est seulement supposée semisimple, on voit qu'on fait ainsi correspondre à tout $i \in I(X)$ un morphisme $\lambda_{i}$ de $B$ dans $B^{*}$ ayant les propriétés qui résultent de ce qui précède. En particulier, pour $i=i_{X}(x)$, le noyau de $\lambda_{i}$ contient toujours celui de $x$ quand $x$ est considéré comme application de $B$ dans $A$.

On dira qu'un élément $i$ de $I(X)$ est non-dégénéré si le noyau de $\lambda_{i}$ se réduit à $\{0\}$; les formules du $n^{\circ} 24$ montrent immédiatement dans les cas (I) et (II) qu'alors $\lambda_{i}$ est un isomorphisme de $B$ sur $B^{*}$, et par suite qu'alors $X$ est isomorphe à son dual $X^{*}$; il s'ensuit que cette conclusion reste valable dans le cas général. Pour qu'il existe des éléments nondégénérés dans $I(X)$, il est donc nécessaire que $X$ soit isomorphe à $X^{*}$; mais cela n'est pas suffisant. Si $x \in X$ et que $i_{X}(x)$ soit non-dégénéré, le noyau de $x$ dans $B$ doit être $\{0\}$; d'après le lemme 8 du $n^{\circ} 17$, cela revient à dire que $x$ est de rang maximal. Autrement dit, si $i$ est non-dégénéré, on a $U(i)=i_{X}^{-1}(\{i\})$; par suite, d'après la proposition $3 \mathrm{du} \mathrm{n}^{\circ} 22$, le groupe $G$ opère alors transitivement sur $i_{X}^{-1}(\{i\})$.

D'autre part, soit $i \in I(X)$; soit $B^{\prime}$ le noyau de $\lambda_{i}$; soit $B^{\prime \prime}$ un supplémentaire de $B^{\prime}$ dans $B$. Désignons par $g^{\prime}$ le sous-groupe de $\operatorname{Aut}(B)$ formé des automorphismes de $B$ qui laissent $B^{\prime}$ et $B^{\prime \prime}$ invariants et induisent l'identité sur $B^{\prime \prime}$. On vérifie immédiatement, dans le cas (I) et dans le cas (II), que $g^{\prime}$ laisse $i$ invariant; identifiant $\operatorname{Aut}(B)$ avec $\operatorname{Aut}(X)$ comme au $\mathrm{n}^{\circ} 24$, on peut donc considérer $g^{\prime}$ comme un sous-groupe de $\operatorname{Aut}(X, i)$; et cette conclusion, une fois vérifiée dans les cas (I) et (II), reste évidemment valable dans le cas général. Avec ces notations, on a alors le résultat suivant :

Proposition 4. Pour que deux éléments de $i_{X}^{-1}(\{i\})$ appartiennent à une même orbite de $G \times g^{\prime}$, il faut et il suffit qu'ils aient même rang.

La condition est évidemment nécessaire. Réciproquement, soient $x_{0}$ et $x_{1}$ deux éléments de $i_{\bar{X}}^{-1}(\{i\})$, et soient $N_{0}$ et $N_{1}$ leurs noyaux dans $B$; d'après ce qui précède, $N_{0}$ et $N_{1}$ sont des sous-modules du noyau $B^{\prime}$ de $\lambda_{i}$ pour la structure de $\boldsymbol{B}$-module à droite de $B$. Mais $\mathcal{B}$ est somme directe des corps $\mathfrak{l}_{v}$; si on décompose $B, B^{\prime}, N_{0}$ et $N_{1}$ en leurs composantes relatives aux $\mathfrak{f}_{v}$, ces composantes sont des espaces vectoriels à droite sur les $\mathfrak{f}_{v}$, et l'hypothèse qu'on a faite sur les rangs de $x_{0}$ et $x_{1}$ équivaut à dire que, pour chaque $v$, les composantes de $N_{0}$ et de $N_{1}$ relatives à $\mathfrak{f}_{v}$ sont des espaces de même dimension sur $\mathfrak{f}_{v}$, contenus tous deux dans la composante correspondante de $B^{\prime}$. Il y a done un automorphisme $v^{\prime}$ de $B^{\prime}$ qui transforme $N_{1}$ en $N_{0}$. Alors l'automorphisme $v$ de $B$ qui coìncide avec $v^{\prime}$ sur $B^{\prime}$ et avec l'identité sur $B^{\prime \prime}$ appartient à $g^{\prime}$ et laisse donc $i$ invariant, de sorte que $x_{0} v$ appartient encore à $i_{X}^{-1}(\{i\})$; et $x_{0} v$ a même noyau $N_{1}$ que $x_{1}$, ce qui implique, d'après le lemme 8 du $n^{\circ} 17$ et la proposition $3 \mathrm{du} \mathrm{n}^{\circ} 22$, que $x_{0} v$ et $x_{1}$ appartiennent à une même orbite de $G$. 
Corollaire. Pour que deux éléments de $i_{X}^{-1}(\{i\})$ appartiennent à une même orbite de $G \times \operatorname{Aut}(X, i)$, il faut et il suffit qu'ils aient même rang.

La condition est évidemment nécessaire, et la proposition 4 montre qu'elle est suffisante.

La proposition 4 et son corollaire montrent en particulier que les groupes $G \times g^{\prime}$ et $G \times \operatorname{Aut}(X, i)$ n'ont qu'un nombre fini d'orbites dans $i_{X}^{-1}(\{i\})$. On observera que la condition qui y intervient est en général plus faible que celle du corollaire de la proposition $3 \mathrm{du}^{\circ} 22$, mais est équivalente à celle-ci dans certains cas. Soient en effet $n=\left(n_{v}\right)$ et $n^{\prime}=\left(n_{p}^{\prime}\right)$ les rangs de $X$ et d'un point $x_{0}$ de $X$, et supposons que, pour tout $v$, on ait, soit $n_{v}^{\prime}=n_{\nu}$, soit $n_{v}^{\prime}=0$ (ce qui aura lieu par exemple si $x_{0}$ est de rang maximal dans $X$, mais aussi chaque fois que $n_{p} \leqslant 1$ quel que soit $v$ ). Il est clair qu'alors on aura $\mathcal{A} x_{0}=\mathcal{A} x_{1}$ chaque fois que $x_{1}$ a même rang que $x_{0}$.

26. Supposons $\mathcal{A}$ absolument semisimple, ce qui revient à dire que, pour tout $\nu$, le centre de $\mathfrak{l}_{v}$ est séparable sur $k$; il en sera toujours ainsi lorsque $k$ est de caractéristique 0 . Alors, si on étend $\mathcal{A}$ par un corps $K \supset k$, l'algèbre étendue $\mathcal{A}_{K}$ est encore semisimple; si en même temps, comme il a été dit au $\mathrm{n}^{\circ} 14$, on étend par $K$ l'involution $\iota$, la trace $\tau$, et les modules sur $\mathcal{A}$, on pourra leur appliquer tout ce qui a été dit ci-dessus. On va maintenant décrire les changements de structure que peut entraîner une telle extension; il suffira pour cela de se placer successivement dans les cas (I) et (II). Pour simplifier, on supposera dans les deux cas que $z_{0}=k$, le cas général se ramenant à celui-ci pour des raisons bien connues; rappelons que $z_{0}$ a été défini, dans le cas $(\mathrm{I})$, comme étant l'ensemble des éléments du centre de $\mathfrak{l}$ qui sont invariants par $\iota$, et, dans le cas (II), comme étant le centre commun de $\mathfrak{l}$ et de $\mathfrak{l}^{\prime}$. Il s'ensuit, dans le cas $(\mathrm{I})$, que le centre $z$ de $\mathfrak{f}$ est, soit $k$, soit de degré 2 sur $k$.

Plaçons-nous d'abord dans le cas (I). Supposons d'abord qu'on ait $z \neq k$ et $K \supset z^{\prime}$; alors $z_{K}$ est somme directe de deux corps isomorphes à $K$, et $\mathcal{A}_{K}$ est somme directe de deux algèbres simples de centre $K$, échangées par l'involution $\iota$; l'extension par $K$ fait donc passer du type (I) au type (II). De plus, $\mathfrak{l}_{K}$ est alors somme directe de deux algèbres simples, anti-isomorphes l'une à l'autre, qu'on pourra écrire $M_{v}(\mathfrak{N})$ et $M_{v}\left(\Re^{\prime}\right)$, où $\Re$ et $\Re^{\prime}$ sont deux algèbres à division de centre $K$, anti-isomorphes l'une à l'autre, de sorte que $\mathcal{A}_{K}$ sera isomorphe à la somme directe de $M_{m_{K}}(\mathscr{\Re})$ et de $M_{m_{K}}\left(\Re^{\prime}\right)$, avec $m_{K}=m \nu$. Si $\delta$ désigne comme précédemment la dimension de $\mathfrak{f}$ sur $k$, et si $\delta_{K}$ désigne celle de $\Re$ sur $K$, on a $\delta=2 \delta_{K} \nu^{2}$. Si $X$ est un $\mathcal{A}$-module à gauche de rang $n, X_{K}$ sera un $\mathcal{A}_{K}$-module de rang $\left(n_{K}, n_{K}\right)$ avec $n_{K}=n \nu$.

Supposons maintenant qu'on soit dans le cas (I) avec $z=k$, ce qui donne $z_{K}=K$, ou bien avec $z \neq k$ et $K \ngtr z$, ce qui entraîne que $z_{K}$ est une extension quadratique de $K$. Alors 
$\mathcal{A}_{K}$ est une algèbre simple; $\mathfrak{l}_{K}$ est de la forme $M_{\nu}(\mathfrak{K})$, où $\mathfrak{\Re}$ est une algèbre à division de centre ${ }_{z_{K}}$, et $\mathcal{A}_{K}$ est alors isomorphe à $M_{m_{K}}(\mathfrak{R})$ avec $m_{K}=m v$. Si $\delta$ et $\delta_{K}$ sont les dimensions de $\mathfrak{l}$ sur $k$ et de $\Re$ sur $K$, respectivement, on a $\delta=\delta_{K} \nu^{2}$. Si $X$ est un $\mathcal{A}$-module à gauche de rang $n, X_{K}$ est un $\mathcal{A}_{K}$-module à gauche de rang $n_{K}=n v$. Enfin on a déjà noté au $n^{\circ} 15$ que $I\left(X_{K}\right)$ s'identifie à $I(X)_{K}$, de sorte que la dimension de $I\left(X_{K}\right)$ sur $K$ est égale à celle de $I(X)$ sur $k$; en appliquant à ces dimensions le lemme 9 du $\mathbf{n}^{\circ} 19$, on en conclut qu'on a

$$
2 \varepsilon_{K}-1=(2 \varepsilon-1) \nu
$$

si $\varepsilon$ est défini comme au n $\mathrm{n}^{\circ} 19$, et si $\varepsilon_{K}$ est le nombre défini de même pour l'algèbre à involution $\mathcal{A}_{K}$ sur $K$.

Si $\mathcal{A}$ est de type (II), $\mathcal{A}_{K}$ sera aussi de type (II); $\mathfrak{f}_{K}$ sera de'la forme $M_{\nu}(\mathfrak{I}), \mathfrak{I}$ étant une algèbre à division de centre $K$; si $\mathfrak{K}^{\prime}$ est anti-isomorphe à $\mathfrak{\Re}, \mathcal{A}_{K}$ sera somme directe de $M_{m_{K}}(\Re)$ et de $M_{m_{K}}\left(\Omega^{\prime}\right)$, avec $m_{K}=m \nu$; on aura $\delta=\delta_{K} \nu^{2}$ si $\delta$ et $\delta_{K}$ sont les dimensions de $\mathfrak{f}$ sur $k$ et de $\mathscr{K}$ sur $K$. Enfin, si $X$ est un $\mathcal{A}$-module à gauche de rang $(p, q), X_{K}$ sera un $\mathcal{A}_{K^{-}}$ module à gauche de rang $\left(p_{K}, q_{K}\right)$ avec $p_{K}=p v, q_{K}=q v$.

On notera que, si on est dans le cas $(I)$ avec $z \neq k$, on a nécessairement $\varepsilon=\frac{1}{2}$; si donc on convient de définir $\varepsilon$ comme ayant la valeur $\frac{1}{2}$ chaque fois que $\mathcal{A}$ est de type (II), la formule (17) restera valable dans tous les cas.

Enfin, il est immédiat que le dual $\left(X_{K}\right)^{*}$ de $X_{K}$ peut toujours s'identifier à $\left(X^{*}\right)_{K}$, et que tout élément de $X$ de rang maximal dans $X$ est encore de rang maximal dans $X_{K}$. On peut vérifier aussi que tout élément de $I(X)$ qui est non-dégénéré dans $I(X)$ l'est encore dans $I\left(X_{K}\right)$.

27. Dans tous les cas qui nous intéressent, $k$ est, soit un corps local, soit un corps de nombres algébriques, soit un corps de fonctions algébriques de dimension 1 sur un corps fini. Si $k$ est l'un de ces corps, il est bien connu que toute algèbre à division $\mathfrak{f}$ sur $k$ sur laquelle il existe une involution laissant invariants tous les éléments du centre de $\mathfrak{f}$ est commutative ou bien est une algèbre de quaternions sur son centre. On en conclut aisément que les algèbres à involution de type (I) sur $k$ se répartissent comme suit en sous-types, dont chacun est caractérisé par la valeur correspondante du nombre $\varepsilon$ défini dans le lemme $9 \mathrm{du} \mathrm{n}^{\circ} 19$ :

$\left(\mathrm{I}_{0}\right) \varepsilon=0$; c'est le cas où on a, avec les notations du $\mathrm{n}^{\circ} 18, \mathfrak{l}=z^{\prime}=z_{0}$ et $\eta=-1$, l'involution $\iota$ étant donc définie par une forme alternée $h$ sur un corps commutatif zo; le groupe $G$ est le groupe symplectique à $m$ variables, $G=S p\left(m, z_{0}\right)$. Comme $h$ doit être non-dégénérée, $m$ est pair. 
$\left(\mathrm{I}_{1}\right) \varepsilon=\frac{1}{4}$; on a $z=z_{0}$, et $\mathfrak{f}$ est une algèbre de quaternions sur $z_{0} ; \xi \rightarrow \xi^{\prime}$ est l'involution "usuelle » sur $\mathfrak{k}$, définie par le fait que $\xi+\xi$ est la trace réduite de $\xi$ sur zo; et $\eta=1$. La forme $h$ est hermitienne (ou, si l'on veut préciser, hermitienne-quaternionique) sur $\mathfrak{l}$, par rapport à l'involution usuelle.

$\left(\mathrm{I}_{2}\right) \varepsilon=\frac{1}{2}$; c'est le cas où ż est une extension quadratique de zo, c'est-à-dire où $\iota$ induit un automorphisme non trivial sur le centre de $\mathcal{A}$; il est d'usage de dire alors que $\iota$ est une involution " de $2^{e}$ espèce ". On peut supposer que $\eta=1$, c'est-à-dire que $h$ est une forme hermitienne.

$\left(\mathrm{I}_{3}\right) \varepsilon=\frac{3}{4}$; on a $z=z_{0}$, f est une algèbre de quaternions sur $z_{0}, \xi \rightarrow \xi$ est l'involution usuelle sur $\mathfrak{f}$, et $\eta=-1$, c'est-à-dire que $h$ est antihermitienne, ou, si l'on veut préciser, antihermitienne-quaternionique, par rapport à l'involution usuelle.

$\left(\mathrm{I}_{4}\right) \varepsilon=1$; on $\mathrm{a} \mathfrak{\mathfrak { l }}=z=z_{0}$ et $\eta=1 ; h$ est une forme quadratique, et $G$ est le groupe ortho. gonal de $h$.

L'effet sur ces sous-types d'une extension par un corps $K \supset k$ se déduit immédiatement de la formule (17) du $n^{\circ} 26$. Comme au $n^{\circ} 26$, supposons pour simplifier qu'on ait $z_{0}=k$. Alors l'extension par $K$ transforme une algèbre de type $\left(\mathrm{I}_{0}\right)$ ou $\left(\mathrm{I}_{4}\right)$ en une algèbre de même type; elle transforme une algèbre de type $\left(I_{1}\right)$ ou $\left(I_{3}\right)$ en une algèbre de même type, si

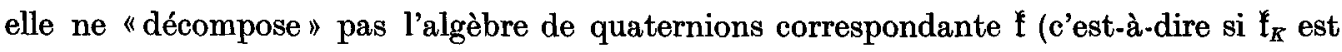
sans diviseurs de zéro), et transforme le type $\left(I_{1}\right)$ en $\left(I_{0}\right)$, et $\left(I_{3}\right)$ en $\left(I_{4}\right)$, dans le cas contraire. Enfin, comme on l'a vu au $n^{\circ} 26$, elle transforme une algèbre de type $\left(I_{2}\right)$ en une algèbre de même type, ou bien de type (II), suivant que $K$ ne contient pas le centre $z$ de l'algèbre en question ou qu'il contient $z$. En particulier, par extension au domaine universel (ou à n'importe quel corps algébriquement clos), les cas $\left(I_{1}\right),\left(I_{2}\right),\left(I_{3}\right)$ se ramènent respectivement à $\left(\mathrm{I}_{\mathbf{0}}\right),(\mathrm{II}),\left(\mathrm{I}_{\mathbf{4}}\right)$, tandis que ceux-ci subsistent tels quels.

28. On a défini le groupe $G$ au $\mathrm{n}^{\circ} 22$ comme le groupe des éléments $u$ de $\mathcal{A}$ tels que $u \cdot u^{\imath}=1$; les résultats ci-dessus permettent dans chaque cas de déterminer la structure de $G$ en tant que groupe algébrique (c'est-à-dire après extension au domaine universel).

Pour cela, convenons de désigner par $\mathcal{A}_{\nu}^{(1)}$, pour chaque $\nu$, le groupe des éléments de $\mathcal{A}_{p}$ dont la norme réduite relativement au centre de $\mathcal{A}_{v}$ est égale à 1 ; soit $\mathcal{A}^{(1)}=\prod \mathcal{A}_{v}^{(1)}$, et soit $G_{1}=G \cap \mathcal{A}^{(1)}$. Dans les cas $\left(\mathrm{I}_{0}\right)$ et $\left(\mathrm{I}_{1}\right)$, on a $G=G_{1} ; G$ est connexe, simple et simplement connexe pour tout $m>0$. Dans les cas $\left(\mathrm{I}_{2}\right)$ et $(\mathrm{II}), G$ est connexe, réductif, et produit (non direct) de son centre et de $G_{1} ; G_{1}$ se réduit à $\{1\}$ pour $m=1, \mathfrak{l}=z$ dans le cas $\left(\mathrm{I}_{2}\right)$, pour $m=1, \mathfrak{l}=\mathfrak{l}^{\prime}=z_{0}$ dans le cas (II), et est connexe, simple et simplement connexe dans tout autre cas. Enfin, dans les cas $\left(\mathrm{I}_{3}\right)$ et $\left(\mathrm{I}_{4}\right), G$, en tant que groupe algébrique, a deux composantes connexes, dont l'une est $G_{1}$; il se trouve d'ailleurs que, dans le cas $\left(\mathrm{I}_{3}\right)$, tout point 
A. WEIL

de $G$, rationnel sur le corps de base, appartient à $G_{1}$; quant à $G_{1}$, il est réduit à $\{1\}$ pour $m=1$ dans le cas $\left(I_{4}\right)$; c'est un tore connexe, de dimension 1 , pour $m=2$ dans le cas $\left(I_{4}\right)$ et pour $m=1$ dans le cas $\left(I_{3}\right)$; il est connexe et semisimple dans tout autre cas; il est même simple pour $m=3$ et pour $m \geqslant 5$ dans le cas $\left(\mathrm{I}_{4}\right)$, et pour $m \geqslant 3$ dans le cas $\left(\mathrm{I}_{3}\right)$. Ces énoncés sont à modifier d'une manière évidente si on ne suppose pas $z_{0}=k$.

On déduit aisément de là, dans chaque cas, la structure du groupe $P s(X / \mathcal{A})$. Dans le cas (II), cette structure est donnée par les résultats du n ${ }^{\circ} 21$, où on a défini un isomorphisme de $P s(X / \mathcal{A})$ sur $G L(p+q, \mathfrak{l})$; dans ce cas, le sous-groupe de $P s(X / \mathcal{A})$ qui correspond à $S L(p+q, \not)$ par cet isomorphisme sera désigné par $P s^{\prime}(X / \mathcal{A})$. Dans le cas $(\mathrm{I})$, il résulte du $n^{\circ} 20$ que $P s(X / \mathcal{A})$ est le groupe analogue à $G$ déterminé par l'algèbre $M_{2 n}(\mathfrak{k})$ munie de l'involution $u \rightarrow e^{\cdot}{ }^{t} u^{\prime} \cdot e^{-1}, e$ étant l'élément de $M_{2 n}(\mathfrak{f})$ donné par (16) et satisfaisant dono $\grave{a ̀ ~}^{t} e^{\prime}=-\eta e$; si $\mathcal{A}$ est de type $\left(\mathrm{I}_{\boldsymbol{p}}\right)$, avec $0 \leqslant \nu \leqslant 4$, cette dernière algèbre est de type $\left(\mathrm{I}_{4-\nu}\right)$. Si $\mathcal{A}$ est de type $\left(\mathrm{I}_{0}\right)$ et qu'on ait $n=1$, on posera $P s^{\prime}(X / \mathcal{A})=\{1\}$; sinon, on désignera par $P s^{\prime}(X / \mathcal{A})$ le groupe (analogue à $\left.G_{1}\right)$ formé des éléments de $P s(X / \mathcal{A})$ dont la norme réduite, prise dans l'algèbre $M_{2 n}(\mathfrak{l})$ relativement à son centre, a la valeur 1 . En vertu de cette définition, $P s^{\prime}(X \mid \mathcal{A})$ se réduit à $\{1\}$ si $\mathcal{A}$ est de type $\left(\mathrm{I}_{0}\right)$ et si $n=1$, ou bien si $\mathcal{A}$ est de type (II) avec $\mathfrak{f}=z_{0}$ et si $p+q=1$, et est connexe et semisimple dans tout autre cas. Si $\mathcal{A}$ est seulement supposée semisimple, on désignera par $P s^{\prime}(X / A)$ le produit direct des groupes analogues relatifs aux composantes simples de $\mathcal{A}$; ce groupe est done toujours, soit connexe et semisimple, soit réduit à $\{1\}$.

29. Voici enfin deux résultats auxiliaires au sujet du sous-groupe parabolique $P(X / \mathcal{A})$ de $P s(X / \mathcal{A})$ qui a été défini au $\mathrm{n}^{\circ} 51$ de [14] et dont la structure dans les cas (I) et (II) a été donnée dans les $\mathrm{n}^{0 s} 20$ et 21 ci-dessus.

Lem Me 11. $P(X / \mathcal{A}) \cdot P s^{\prime}(X / \mathcal{A})$ est un sous-groupe d'indice fini $\operatorname{de} P s(X / \mathcal{A})$.

Il suffit naturellement de le vérifier quand $\mathcal{A}$ est de type (I) ou (II). Dans le cas (II), d'après le $\mathrm{n}^{\circ} 21$, le premier groupe est même égal au second. Quand $\mathcal{A}$ est de type $\left(\mathrm{I}_{0}\right)$ avec $n>1$, ou bien de type $\left(I_{1}\right),\left(I_{3}\right)$ ou $\left(I_{4}\right), P s^{\prime}(X / \mathcal{A})$ coïncide avec $P s(X / \mathcal{A})$ ou bien est d'indice 2 dans celui-ci. Quand $\mathcal{A}$ est de type $\left(\mathrm{I}_{0}\right)$ avec $n=1, P(X / A)$ est d'indice 2 dans

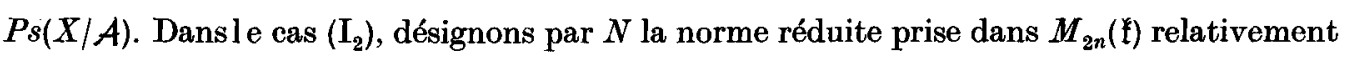
à $\mathfrak{z}$, de sorte que $P s^{\prime}(X / \mathcal{A})$ est le noyau de l'homomorphisme de $P s(X / \mathcal{A})$ dans le groupe multiplicatif de $z$ qui est induit par $N$; si $v$ est l'indice de l'image de $P(X / A)$ dans celle de $P s(X / \mathcal{A})$ par cet homomorphisme, $\nu$ sera aussi l'indice de $P(X / \mathcal{A}) \cdot P s^{\prime}(X / \mathcal{A})$ dans $P s(X / \mathcal{A})$. Mais, d'après la proposition 8 de [14], $\mathrm{n}^{\circ} 51$, tout élément de $P s(X / \mathcal{A})$ appartient à la classe bilatère par rapport à $P(X / \mathcal{A})$ définie par un élément de la forme $s_{1}=d^{\prime}\left(\gamma_{1}\right) \otimes e_{2}$, où $\otimes$ 
se rapporte à une décomposition en somme directe $X=X_{1} \oplus X_{2}$ et où $\gamma_{1}$ est un isomorphisme de $X_{1}^{*}$ sur $X_{1}$ qu'on peut choisir à volonté. Il est facile alors de voir que, si $X_{1}$ et $X_{2}$ sont donnés, on peut choisir $\gamma_{1}$ de manière que $N\left(s_{1}\right)=1$; par suite, dans ce cas, les deux groupes dont il s'agit coïncident.

Lемме 12. Soit $\Delta_{P}$ le module algébrique du groupe $P=P(X / \mathcal{A})$. Soient $g \in Q(X / \mathcal{A})$, $\lambda \in \operatorname{Aut}(X)$ et $p=t(g) d(\lambda)$; et soit $\Delta(\lambda)$ le déterminant, par rapport à une base quelconque de $Q(X / \mathcal{A})$ sur $k$, de l'automorphisme $f \rightarrow f \circ \lambda$ de $Q(X / \mathcal{A})$. Alors on a $\Delta_{P}(p)=\Delta(\lambda)^{-1}$.

En effet, si $p=t(g) d(\lambda)$ est un élément générique de $P$, et si $d g, d \lambda$ sont des jauges invariantes sur l'espace vectoriel $Q(X / \mathcal{A})$ et sur $\operatorname{Aut}(X)$, respectivement, $d p=d g \wedge d \lambda$ est une jauge invariante à droite sur $P$. La conclusion suit immédiatement de là et des résultats du $n^{\circ}$ 4. Bien entendu, $f \circ \lambda$ désigne ici la forme quadratique $x \rightarrow f(x \lambda)$, qui a été notée $f^{\lambda^{-1}}$ dans [14].

\section{Le problème local}

30. Avant d'aborder l'objet propre de ce Chapitre, on va établir encore un résultat auxiliaire. Soit $G$ un groupe algébrique unimodulaire, défini sur $k$; soient $P$ et $U$ des sousgroupes algébriques de $G$, définis aussi sur $k$, tels que $P \cap U=\{e\}$ et que l'algèbre de Lie de $G$ soit somme directe de celles de $P$ et de $U$. Alors $(p, u) \rightarrow p u$ est un $k$-isomorphisme de $P \times U$ sur une partie $k$-ouverte de $G$ (au sens de la topologie de Zariski); pour $g=p u \in P \cdot U$ on pourra donc écrire $p=\varpi(g), u=\sigma(g)$, où $\varpi, \sigma$ sont des morphismes de $P \cdot U$ dans $P$ et dans $U$, respectivement. Soient $d g, d p, d u$ des jauges invariantes à gauche dans $G, P$ et $U$, respectivement. Dans $P \cdot U$, on pourra écrire $d g=f(p, u) d p \wedge d u$, où $f$ est une fonction partout finie et non nulle dans $P \times U$; on aura alors $f\left(p^{\prime} p, u u^{\prime}\right)=f(p, u) \Delta_{U}\left(u^{\prime}\right)^{-1}$, et par suite $f$ ne diffère de $\Delta_{U}^{-1}$ que par un facteur constant. Si on suppose que $U$ soit aussi unimodulaire, on aura $d g=d p \wedge d u$ à un facteur constant près qu'on pourra supposer égal à 1 .

Quel que soit $g_{0} \in G$, l'ensemble $U_{0}=U \cap P \cdot U g_{0}$ est une partie $k$-ouverte de $U$. Pour $u \in U_{0}$, posons $p^{\prime}=\sigma\left(u g_{0}^{-1}\right), u^{\prime}=\sigma\left(u g_{0}^{-1}\right)$; on aura $u g_{0}^{-1}=p^{\prime} u^{\prime}$, d'où $p^{\prime-1} u=u^{\prime} g_{0}$ et par suite $p^{\prime}=\varpi\left(u^{\prime} g_{0}\right)^{-1}, u=\sigma\left(u^{\prime} g_{0}\right)$ et $u^{\prime} \in U_{0}^{\prime}=U \cap P \cdot U g_{0}^{-1}$; il s'ensuit que $u \rightarrow u^{\prime}$ est un isomorphisme de $U_{0}$ sur $U_{0}^{\prime}$. On a alors, dans $P \cdot U_{0}, d\left(p u g_{0}^{-1}\right)=d\left(p p^{\prime} u^{\prime}\right)$, c'est-à-dire

$$
d p \wedge d u=d\left(p p^{\prime}\right) \wedge d u^{\prime}=\Delta_{P}\left(p^{\prime}\right) d p \wedge d u^{\prime}
$$

et par suite

$$
d u=\Delta_{P}\left(p^{\prime}\right) d u^{\prime}
$$


Supposons maintenant que $k$ soit un corps local, et aussi que $G$ soit un groupe réductif et $P$ un sous-groupe "parabolique " (non nécessairement minimal) de $G$, ce qui revient à dire que $G / P$ est isomorphe à une variété projective. Pour simplifier les notations, on écrira $G, P, U$ au lieu de $G_{k}, P_{k}, U_{k}$; on notera $|d g|,|d p|,|d u|$ les mesures de Haar sur ces groupes déduites des jauges $d g, d p, d u$ comme il a été dit au $n^{\circ} 3$ du Chapitre I. Comme d'habitude, pour $x \in k$, on notera $|x|$ le module de $x$ dans $k$. Avec ces notations, $G, P$ et $U$ sont des variétés $k$-analytiques, et $G / P$ est une variété $k$-analytique compacte; il en est de même de l'espace $V=P \backslash G$ des classes à gauche suivant $P$ dans $G$.

LEM $\left|\Delta_{P}\right| \cdot \psi$; soit $f_{0}$ une fonction continue, partout $>0$ sur $G$, telle que $f_{0}(p g)=\psi(p) f_{0}(g) q u e l s q u$ soient $p \in P, g \in G$. Alors, pour que $\int_{U} f_{0}(u)|d u|<+\infty$, il faut que, pour tout $g_{0} \in G$, il y ait un voisinage $U^{\prime}$ de e dans $U$ tel que $\psi^{\prime}\left(\varpi\left(u g_{0}\right)^{-1}\right)$ soit intégrable dans $U^{\prime} \cap P \cdot U g_{0}^{-1}$, et il suffit que toute classe à gauche suivant $P$ dans $G$ contienne un élément $g_{0}$ qui possède cette propriété.

Comme $V=P \backslash G$ est compact, il faut et il suffit, pour que $f_{0}$ soit intégrable sur $U$, que tout point de $V$ (c'est-à-dire toute classe à gauche $P g_{0}$ suivant $P$ ) ait un voisinage $V^{\prime}$ tel que $f_{0}$ soit intégrable dans $U \cap V^{\prime}$; comme $U g_{0}$ est transversale à $P g_{0}$ en $g_{0}$, on peut d'ailleurs toujours supposer que $V^{\prime}$ est pris de la forme $V^{\prime}=P \cdot U^{\prime} g_{0}$, où $U^{\prime}$ est un voisinage de $e$ dans $U$. La condition qu'on vient d'énoncer revient alors à dire que $f_{0}(u)|d u|$ est intégrable dans $U \cap P \cdot U^{\prime} g_{0}$. En faisant le changement de variable $u=\sigma\left(u^{\prime} g_{0}\right)$, cela s'écrit aussi, d'après (18) :

$$
\int_{U_{1}^{\prime}} f_{0}\left(p^{\prime} u^{\prime} g_{0}\right)\left|\Delta_{P}\left(p^{\prime}\right)\right| \cdot\left|d u^{\prime}\right|<+\infty
$$

avec $p^{\prime}=\varpi\left(u^{\prime} g_{0}\right)^{-1}$ et $U_{1}^{\prime}=U^{\prime} \cap P \cdot U g_{0}^{-1}$. Si on tient compte des hypothèses faites sur $f_{0}$, on obtient le résultat annoncé.

Il est bien connu que, si $\psi$ est donnée, il existe toujours une fonction $f_{0}$ ayant les propriétés énoncées dans le lemme 13 (cf. [4], § 2, n $\mathrm{n}^{\circ}$, prop. 7). Par suite, si la condition du lemme 13 est satisfaite, et si $C$ est une partie compacte de $G$ telle que $G=P \cdot C$, l'intégrale $\int_{U} f(u)|d u|$ sera absolument convergente, uniformément sur tout ensemble de fonctions continues $f$ sur $G$, uniformément bornées sur $C$ et satisfaisant à $f(p g)=\psi(p) f(g)$ quels que soient $p \in P$ et $g \in G$. En effet, si $|f| \leqslant M$ sur $C$, et si $m=\inf _{C}\left(f_{0}\right)$, on aura $\left|f_{0}^{-1} f\right| \leqslant m^{-1} M$ sur $C$, donc aussi en tout point de $G$ puisque $f_{0}^{-1} f$ est constante sur les classes à gauche suivant $P$ dans $G$.

31. Dans tout le reste de ce chapitre, $k$ désignera un corps local de caractéristique autre que $2, \mathcal{A}$ une algèbre semisimple sur $k$ munie d'une involution, et $X$ un. A-module à gauche. 
On conservera les hypothèses et notations de [14], Chap. II, $\mathrm{n}^{\circ} 24$, de [14], Chap. V, $\mathrm{n}^{\text {os }}$ 49-51, et du Chapitre II ci-dessus. Pour abréger, on écrira $Q, P s, P$ au lieu de $Q(X / \mathcal{A})$, $P s(X / \mathcal{A}), P(X / \mathcal{A})$.

Dans le présent Chapitre, on se propose principalement d'appliquer la proposition 1 du Chapitre $\mathrm{I}, \mathrm{n}^{\circ} 2$, au cas où $X$ est comme ci-dessus, où $G=I(X)$, et où on prend pour $f$ l'application $i_{X}$ de $X$ dans $I(X)$. Pour cela, il faut avant tout déterminer dans quels cas $i_{X}$ satisfait à la condition $(\mathrm{A})$ de cette proposition.

On posera donc, pour $\Phi \in S(X)$ et $i^{*} \in I(X)^{*}$ :

$$
F_{\Phi}^{*}\left(i^{*}\right)=\int_{X} \Phi(x) \chi\left(\left[i_{X}(x), i^{*}\right]\right)|d x|
$$

Identifiant $I(X)^{*}$ avec $Q=Q(X / \mathcal{A})$ comme il a été dit au $\mathbf{n}^{\circ} 15$, on pourra encore écrire, pour $q \in Q$ :

$$
F_{\Phi}^{*}(q)=\int_{X} \Phi(x) \chi(q(x))|d x|
$$

Dire que $i_{X}$ satisfait à la condition (A) équivaut alors à dire que la fonction $F_{\Phi}^{*}$ ainsi définie est intégrable sur $Q$, et cela uniformément par rapport à $\Phi$ sur toute partie compacte de $S(X)$; quand il en sera ainsi, on dira que $\mathcal{A}$ et $X$ ont la propriété (A) ou encore satisfont à la condition (A).

Pour abréger, nous nous bornerons au cas où $X$ est isomorphe à son dual $X^{*}$. Nous démontrerons entre autres le résultat suivant :

Proposition 5. Si $X$ est isomorphe à son dual $X^{*}$, il faut et il suffit, pour que $\mathcal{A}$ et $X$ aient la propriété (A), qu'il en soit ainsi de chaque composante simple de $\mathcal{A}$ et de la composante correspondante de $X$. Pour que $\mathcal{A}$ et $X$ aient la propriété (A), il suffit qu'on ait $m \geqslant 2 n+4 \varepsilon-2$ si $\mathcal{A}$ est de type (I) et $X$ de rang $n$, ou $m \geqslant 2 p$ si $A$ est de type (II) et $X$ de rang $(p, p)$.

Soit $\gamma$ un isomorphisme de $X^{*}$ sur $X$. Avec les notations de [14], Chap. III, on aura

$$
F_{\Phi}^{*}(q)=|\gamma|^{\frac{1}{2}} \mathbf{d}^{\prime}(\gamma) \mathbf{t}(q) \Phi(0)
$$

Mais, en vertu de la définition du groupe $M p(X / \mathcal{A})$ et de sa projection canonique $\pi$ sur $P s=P s(X / A)$ (cf. [14], Chap. III, et Chap. V, $\mathrm{n}^{\circ} 51$ ), on peut définir sur $P s$ une fonction continue $f_{\Phi}$ en posant, pour tout $\mathrm{S} \in M p(X / \mathcal{A})$ :

$$
f_{\Phi}(\pi(\mathbf{S}))=\left|\mathbf{S} \mathbf{d}^{\prime}(\gamma) \Phi(0)\right|
$$

Pour tout élément $p=t(q) d(\lambda)$ de $P$, avec $q \in Q, \lambda \in \operatorname{Aut}(X)$, posons $\psi(p)=|\lambda|_{X}^{\frac{1}{x}}$, où $|\lambda|_{x}$ 
désigne comme d'habitude le module de l'automorphisme $\lambda$ de $X$. Posons aussi $\Phi^{\prime}=\mathbf{S d}^{\prime}(\gamma) \Phi$, et $s=\pi(\mathrm{S})$; d'après la définition $\operatorname{de~} \mathrm{t}(q)$ et de $\mathrm{d}(\lambda)$ aux $\mathrm{n}^{\text {os }} 13$ et 34 de [14], on aura

et par suite

$$
\mathbf{t}(q) \mathbf{d}(\lambda) \Phi^{\prime}(x)=|\lambda|_{X}^{\frac{1}{\prime}} \Phi^{\prime}(x \lambda) \chi(q(x))
$$

$$
f_{\Phi}(p s)=\left|\mathbf{t}(q) \mathbf{d}(\lambda) \Phi^{\prime}(0)\right|=|\lambda| \frac{1}{X} \cdot\left|\Phi^{\prime}(0)\right|=\psi(p) f_{\Phi}(s) .
$$

Soit d'autre part $U$ le sous-groupe de $P s$ formé des éléments $t^{\prime}\left(q^{\prime}\right)$ avec $q^{\prime} \in Q^{\prime}=Q\left(X^{*} / \mathcal{A}\right)$; pour $G=P s$, les sous-groupes $P$ et $U$ ont bien les propriétés énoncées ci-dessus au $\mathrm{n}^{\circ} 30$. De plus, $q \rightarrow-q \circ \gamma$ est un isomorphisme de $Q$ sur $Q^{\prime}$, de sorte que $q \rightarrow t^{\prime}(-q \circ \gamma)$ est un isomorphisme de $Q$ sur $U$. On vérifie aisément d'ailleurs qu'on a, quel que soit $q \in Q$ :

$$
t^{\prime}(-q \circ \gamma)=d^{\prime}(\gamma) t(q) d^{\prime}(\gamma)^{-1}
$$

Il s'ensuit qu'on a, dans ces conditions :

$$
\left|F_{\Phi}^{*}(q)\right|=|\gamma|^{\frac{1}{2}} f_{\Phi}\left(t^{\prime}(-q \circ \gamma)\right)
$$

ce qui montre que l'intégrabilité de $F_{\Phi}^{*}$ sur $Q$ équivaut à celle de $f_{\Phi}$ sur $U$. Compte tenu de la remarque finale du $n^{\circ} 30$, on voit donc que la condition (A) pour $\mathcal{A}$ et $X$ équivant à la condition du lemme $13 \mathrm{du} \mathrm{n}^{\circ} 30$ pour les groupes $G=P s, P$ et $U$ et la représentation $\psi$ de $P$ tels qu'on vient de les définir.

32. Le module $\Delta_{P}$ de $P$, qui figure dans la condition du lemme 13, est donné par le lemme $12 \mathrm{du} \mathrm{n}^{\circ} 29$. D'autre part, dans cette condition, on peut prendre pour représentants des classes à gauche suivant $P$ ceux qui sont donnés par la proposition 8 de [14], $\mathrm{n}^{\circ} 51$. D'après celle-ci, pour obtenir de tels représentants, on doit choisir de toutes les manières possibles un sous-module $X_{1}$ de $X$, isomorphe à son dual $X_{1}^{*}$; pour chaque $X_{1}$, on choisit un isomorphisme $\gamma_{1}$ de $X_{1}^{*}$ sur $X_{1}$ et un supplémentaire $X_{2}$ de $X_{1}$ dans $X$; enfin on choisit, de toutes les manières possibles, un élément $q_{1}$ de $Q\left(X_{1} / \mathcal{A}\right)$, et, pour chaque $q_{1}$, on écrit

$$
s_{0}=d^{\prime}\left(\gamma_{1}\right) t\left(q_{1}\right) \otimes e_{2}
$$

Les $s_{0}$ forment le système de représentants en question.

Avec les notations du $\mathrm{n}^{\circ} 30$, il reste à calculer $\psi^{\prime}\left(p^{\prime}\right)$ pour $p^{\prime}=\varpi\left(u s_{0}\right)^{-1}, u \in U \cap P \cdot U s_{0}^{-1}$, $s_{0}$ étant donné par (19). Puisque $u \in U$, on peut l'écrire sous la forme $u=t^{\prime}\left(q^{\prime}\right)$ avec $q^{\prime} \in Q^{\prime}$. Par définition de $\varpi$, il y aura alors $q^{\prime \prime} \in Q^{\prime}$ tel que l'on ait

$$
t^{\prime}\left(q^{\prime}\right) s_{0}=p^{\prime-1} t^{\prime}\left(q^{\prime \prime}\right)
$$

Ecrivons $p^{\prime}=t(q) d(\lambda)$ avec $q \in Q, \lambda \in \operatorname{Aut}(X)$, et explicitons les éléments du groupe $S p(X / \mathcal{A})$ qui correspondent aux deux membres de (20). Si on note 


$$
\sigma_{0}=\left(\begin{array}{ll}
\alpha_{0} & \beta_{0} \\
\gamma_{0} & \delta_{0}
\end{array}\right)
$$

celui qui correspond à $s_{0}$, et qu'on désigne par $\varrho^{\prime}$ le morphisme symétrique de $X^{*}$ dans $X$ associé à la forme quadratique $q^{\prime}$ sur $X^{*}$, on trouve ainsi que (20) entraîne en particulier la relation $\lambda^{*}=\varrho^{\prime} \beta_{0}+\delta_{0}$.

Désignons encore par $\varrho_{1}$ le morphisme symétrique de $X_{1}$ dans $X_{1}^{*}$ associé à $q_{1}$; pour les décompositions $X=X_{1} \oplus X_{2}, X^{*}=X_{1}^{*} \oplus X_{2}^{*}$ de $X$ et $X^{*}$ en sommes directes, $\beta_{0}$ et $\delta_{0}$ s'écrivent sous forme matricielle comme suit :

$$
\beta_{0}=\left(\begin{array}{cc}
-\gamma_{1}^{*-1} & 0 \\
0 & 0
\end{array}\right), \quad \delta_{0}=\left(\begin{array}{cc}
\gamma_{1} \varrho_{1} & 0 \\
0 & 1
\end{array}\right)
$$

Si nous écrivons $\varrho^{\prime}$ sous forme matricielle pour cette même décomposition de $X$ et de $X^{*}$, on aura, puisque $\varrho^{\prime}$ est symétrique :

$$
\varrho^{\prime}=\left(\begin{array}{cc}
\varrho_{1}^{\prime} & \tau \\
\tau^{*} & \varrho_{2}^{\prime}
\end{array}\right)
$$

où $\varrho_{1}^{\prime}, \varrho_{2}^{\prime}$ sont des morphismes symétriques de $X_{1}^{*}$ dans $X_{1}$ et de $X_{2}^{*}$ dans $X_{2}$, respectivement, et où $\tau$ est un morphisme de $X_{1}^{*}$ dans $X_{2}$. On aura alors

$$
\lambda^{*}=\left(\begin{array}{cc}
\gamma_{1} \varrho_{1}-\varrho_{1}^{\prime} \gamma_{1}^{*-1} & 0 \\
-\tau^{*} \gamma_{1}^{*-1} & 1
\end{array}\right)
$$

ou, ce qui revient au même, et en posant de plus $\bar{\varrho}_{1}=\gamma_{1} \varrho_{1} \gamma_{1}^{*}$ :

$$
\lambda=\left(\begin{array}{cc}
\gamma_{1}^{-1}\left(\bar{\varrho}_{1}-\varrho_{1}^{\prime}\right) & -\gamma_{1}^{-1} \tau \\
0 & 1
\end{array}\right)
$$

Ici $\bar{\varrho}_{1}$ est le morphisme symétrique de $X_{1}^{*}$ dans $X_{1}$ associé à la forme quadratique $\bar{q}_{1}=q_{1} \circ \gamma_{1}$ sur $X_{1}^{*}$, de sorte que $\bar{\varrho}_{1}-\varrho_{1}^{\prime}$ est un morphisme symétrique de $X_{1}^{*}$ dans $X_{1}$; si celui-ci est un isomorphisme, (22) définit un automorphisme $\lambda$ de $X_{1}$. De plus, quand cette dernière condition est satisfaite, il est aisé de voir, en appliquant la proposition 1 de [14], $\mathrm{n}^{\circ} 7$, ̀̀ $s=d(\lambda) t^{\prime}\left(q^{\prime}\right) s_{0} d^{\prime}(\gamma)$, qu'on peut déterminer $q$ et $q^{\prime \prime}$ de manière à satisfaire à $(20)$, ce qui montre qu'alors $u=t^{\prime}\left(q^{\prime}\right)$ appartient à $P \cdot U s_{0}^{-1}$.

Toujours avec les mêmes notations, on a alors, d'après ce qui précède :

$$
\psi^{\prime}\left(p^{\prime}\right)=\psi^{\prime}\left(\varpi\left(u s_{0}\right)^{-1}\right)=|\lambda|_{X}^{\frac{1}{2}}|\Delta(\lambda)|^{-1} .
$$

Donc, pour que (A) soit satisfaite, il faut et il suffit que cette expression, considérée comme fonction de $q^{\prime}$, soit intégrable au voisinage de 0 dans $Q^{\prime}$, et cela pour tout $s_{0}$ de la forme (19). 
33. Cette question se ramène évidemment aux questions analogues relatives aux composantes simples de $\mathcal{A}$; cela démontre la première assertion de la proposition 5 . Supposons maintenant que $\mathcal{A}$ soit de type (I); alors, avec nos notations habituelles, Aut $(X)$ s'identifie à $G L(n, \mathfrak{f})$, c'est-à-dire au groupe des éléments inversibles de $M_{n}(\mathfrak{f})$. On sait que tout caractère de ce groupe, rationnel sur $k$ (au sens de la théorie des groupes algébriques) est de la forme $\lambda \rightarrow \nu_{n}(\lambda)^{N}$, où $N$ est un entier et où $\nu_{n}$ est la norme réduite prise dans $M_{n}(\mathfrak{f})$ relativement à $k$. Il en sera donc ainsi, en particulier, de $\Delta(\lambda)$, et aussi du déterminant $D(\lambda)$ de l'automorphisme $\lambda$ de $X$, pris par rapport à une base quelconque de $X$ sur $k$. Pour achever de déterminer ceux-ci, il suffit de les calculer, ainsi que $v_{n}(\lambda)$, dans le cas où $\lambda$ est de la forme $t \cdot 1$, avec $t \in k, t \neq 0$. Si $\delta$ désigne comme toujours la dimension de $\mathfrak{f}$ sur $k$, et $\alpha^{2}$ celle de $\mathfrak{l}$ sur son centre $\mathfrak{z}$, on aura, pour ce choix de $\lambda$ :

$$
\nu_{n}(\lambda)=t^{\delta n / \alpha}, \quad D(\lambda)=t^{\delta m n},
$$

et, d'après le lemme 9 du $n^{\circ} 19$ :

$$
\Delta(\lambda)=t^{\delta n(n+2 \varepsilon-1)} .
$$

On a donc, quel soit $\lambda \in \operatorname{Aut}(X)$ :

$$
D(\lambda)=v_{n}(\lambda)^{\alpha m}, \quad \Delta(\lambda)=\nu_{n}(\lambda)^{\alpha(n+2 \varepsilon-1)} .
$$

Comme on a d'ailleurs $|\lambda|_{X}=|D(\lambda)|$, (23) s'écrit donc:

$$
\psi^{\prime}\left(p^{\prime}\right)=\left|\nu_{n}(\lambda)\right|^{\alpha(m-2 n-4 \varepsilon+2) / 2} .
$$

Mait $\lambda$ est donné par (22) chaque fois que (22) définit un automorphisme de $X$. Posons $\varrho=\bar{\varrho}_{1}-\varrho_{1}^{\prime}$; si $n_{1}$ est le rang du module $X_{1}$, $\varrho$ est une matrice $\eta$-hermitienne à $n_{1}$ lignes et $n_{1}$ colonnes, et on a :

$$
\nu_{n}(\lambda)=\nu_{n_{1}}\left(\gamma_{1}\right)^{-1} \cdot \nu_{n_{1}}(\varrho)
$$

Nous avons à exprimer que $\psi^{\prime}\left(p^{\prime}\right)$, considéré comme fonction de $q^{\prime}$, ou, ce qui revient au même, de $\varrho^{\prime}$, ou, ce qui revient encore au même d'après $(21)$, de $\left(\varrho_{1}^{\prime}, \varrho_{2}^{\prime}, \tau\right)$, est intégrable au voisinage de 0 dans le complémentaire de l'ensemble des points où $v_{n_{1}}(\varrho)=0$. Cela équivaut à dire que $\psi^{\prime}\left(p^{\prime}\right)$, considéré comme fonction de $\varrho$, est intégrable dans ce complémentaire au voisinage de $\bar{\varrho}_{1}$. En d'autres termes, on a démontré ce qui suit :

Lемме 14. Soient $\mathcal{A}$ de type $(\mathrm{I})$, et $X$ de rang $n$. Pour que $\mathcal{A}$ et $X$ aient la propriété (A), il faut et il suffit que, pour tout $n_{1} \leqslant n$, la fonction

$$
\varphi(\varrho)=\left|\nu_{n_{1}}(\varrho)\right|^{\alpha(m-2 n-4 \varepsilon+2) / 2}
$$


soit localement intégrable au voisinage de tout point dans l'espace des matrices $\eta$-hermitiennes $\grave{a} n_{1}$ lignes et $n_{1}$ colonnes sur $\mathfrak{l}$.

Comme cette fonction est partout continue pour $m \geqslant 2 n+4 \varepsilon-2$, la seconde assertion de la proposition 5 est contenue dans ce résultat.

Soient maintenant $\mathcal{A}$ de type (II), et $X$ de rang $(p, p)$. Avec nos notations habituelles, $X$ s'identifie à la somme directe de $M_{m, p}\left(\mathfrak{f}^{\prime}\right)$ et de $M_{m, p}\left(\mathfrak{l}^{\prime}\right)$, et par suite $\operatorname{Aut}(X)$ à

$$
G L(p, \mathfrak{l}) \times G L\left(p, \mathfrak{l}^{\prime}\right)
$$

Soient $v_{p}, v_{p}^{\prime}$ les normes réduites prises relativement à $k$ dans $M_{p}(\mathfrak{f})$ et $M_{p}\left(\mathfrak{f}^{\prime}\right)$,respectivement. En raisonnant comme plus haut, on voit aisément que, si $\lambda=(v, w)$ appartient à $\operatorname{Aut}(X)$, on a :

$$
|\lambda|_{X}=\left|v_{p}(v) v_{p}^{\prime}(w)\right|^{\alpha m}, \quad|\Delta(\lambda)|=\left|v_{p}(v) \nu_{p}^{\prime}(w)\right|^{\alpha n},
$$

où on a de nouveau désigné par $\alpha^{2}$ la dimension de $\mathfrak{f}$ sur son centre. Procédant alors comme dans le cas (I), on obtient le résultat suivant :

Lemme 15. Soient $\mathcal{A}$ de type (II), et $X$ de rang $(p, p)$. Pour que $\mathcal{A}$ et $X$ aient la propriété (A), il faut et il suffit que, pour tout $p_{1} \leqslant p$, la fonction

$$
\varphi(r)=\left|\nu_{p_{1}}(r)\right|^{\alpha(m-2 p)}
$$

soit localement intégrable au voisinage de tout point dans l'espace $M_{p_{1}}(\mathfrak{t})$.

La dernière assertion de la proposition 5 est un cas particulier de ce lemme.

34. En fait, une analyse un peu plus poussée montrerait que, dans les conditions du lemme 14, la fonction $\left|\nu_{n}(\varrho)\right|^{\alpha s}$ est localement intégrable au voisinage de tout point, dans l'espace des éléments $\eta$-hermitiens de $M_{n}(\mathfrak{f})$, chaque fois que $s>-\varepsilon$ si $\varepsilon \neq 0$, chaque fois que $s>-\frac{1}{2}$ si $\varepsilon=0$, et qu'elle cesse de l'être au voisinage de 0 si $s$ ne satisfait pas à cette condition. De même, dans les conditions du lemme 15, la condition d'intégrabilité locale de $\left|v_{p}(r)\right|^{\alpha s}$ est $s>-1$. Enfin, si on cesse de supposer que $X$ soit isomorphe à son dual, on trouve que la première assertion de la proposition 5 reste vraie; et, dans le cas (II), on trouve que (A) équivaut à $m \geqslant p+q$ si $X$ est de rang $(p, q)$.

Convenons de dire que $\mathcal{A}$ et $X$ satisfont à la condition ( $\left.\mathbf{A}^{\prime}\right)$ si $\varepsilon=0$ et $m>2 n-3$ ou bien si $\varepsilon \neq 0$ et $m>2 n+2 \varepsilon-2$ dans le cas (I), si $m \geqslant p+q$ dans le cas (II), et, dans le cas général, si ces dernières conditions sont satisfaites par chaque composante simple de $\mathcal{A}$ et par la composante correspondante de $X$. Il résulte de ce qu'on vient de dire qu'en fait (A) est toujours équivalente à ( $\left.\mathbf{A}^{\prime}\right)$. D'ailleurs, si $\mathcal{A}$ est de type $(\mathbf{I}),\left(\mathbf{A}^{\prime}\right)$ ne diffère de la 4-652922. Acta mathematica. 113. Imprimé le 26 fóvrier 1965. 
condition suffisante fournie par la proposition 5 que dans les cas $\left(\mathrm{I}_{3}\right),\left(\mathrm{I}_{4}\right) ;\left(\mathrm{A}^{\prime}\right)$ donne $m \geqslant 2 n$ dans le cas $\left(\mathrm{I}_{3}\right)$ et $m \geqslant 2 n+1$ dans le cas $\left(\mathrm{I}_{4}\right)$, tandis que la proposition 5 donnerait seulement $m \geqslant 2 n+1$ et $m \geqslant 2 n+2$, respectivement.

35. Dans le reste de ce Chapitre, nous supposerons une fois pour toutes que la condition (A) est satisfaite, c'est-à-dire qu'on a le droit d'appliquer à $i_{X}$ la proposition 1 du $\mathrm{n}^{\circ} 2$. On peut donc, d'une manière et d'une seule, définir pour tout $i \in I(X)$ une mesure $\mu_{i}$, de support contenu dans $i_{X}^{-1}(\{i\})$, de telle sorte que, pour toute fonction $\Phi$ continue à support compact sur $X$, la fonction $F_{\Phi}(i)=\int \Phi d \mu_{i}$ soit continue sur $I(X)$ et satisfasse à $\int \Phi|d x|=\int F_{\Phi}|d i|$.

Avec les notations du Chapitre II, $\mathrm{n}^{\circ} 24$, soient alors $u \in G, v \in \operatorname{Aut}(X)$. On a $i_{X}(u x v)=$ $i_{X}(x) \bar{v}$, où $\bar{v}$ est l'automorphisme de $I(X)$ déduit de $v$ par transport de structure. Dans la formule

$$
\int_{X} \Phi(x)|d x|=\int_{I(X)}\left(\int \Phi(x) d \mu_{i}(x)\right)|d i|
$$

remplaçons $\Phi$ par la fonction $\Psi^{\circledR}$ donnée par

$$
\Psi(x)=\Phi\left(u^{-1} x v^{-1}\right)
$$

puis remplaçons $x$ par uxv dans les deux membres, et $i$ par $i \bar{v}$ au second membre. Cela donne

$$
|u|_{X} \cdot|v|_{X} \int_{X} \Phi(x)|d x|=|\bar{v}|_{I(X)} \int_{I(X)}\left(\int \Phi(x) d \mu_{i \bar{v}}(u x v)\right)|d i|
$$

où $|u|_{X},|v|_{X},|\bar{v}|_{I(X)}$ désignent bien entendu les modules de $u, v, \bar{v}$ dans $X, X$ et $I(X)$, respectivement. En vertu de la propriété d'unicité des $\mu_{i}$ qu'on a rappelée plus haut, on en conclut:

$$
d \mu_{i \bar{v}}(u x v)=|u|_{X} \cdot|v|_{X} \cdot|\bar{v}|_{I(X)}^{-1} \cdot d \mu_{i}(x) .
$$

En particulier, pour tout $i \in I(X), \mu_{i}$ est relativement invariante par $G \times \operatorname{Aut}(X, i)$, et on a, pour $\mu=\mu_{i}, u \in G, v \in \operatorname{Aut}(X, i)$ :

$$
d \mu(u x v)=|u|_{X} \cdot|v|_{X} \cdot|\bar{v}|_{I(X)}^{-1} \cdot d \mu(x)
$$

Mais le corollaire de la proposition 4 du Chapitre II, $\mathbf{n}^{\circ} 25$, montre que $G \times \operatorname{Aut}(X, i) n^{\prime a}$ qu'un nombre fini d'orbites dans $i_{X}^{-1}(\{i\})$; cela permet d'appliquer la remarque élémentaire suivante, qu'on formulera sous forme de lemme :

LEм Е 16. Soit $G$ un groupe localement compact, dénombrable à l'infini, opérant sur un espace localement compact $E$; supposons que $G$ 'ait qu'un nombre fini d'orbites distinctes 
$E_{\alpha}$ dans $E$. Alors chaque orbite $E_{\alpha}$ est relativement ouverte dans son adhérence et est isomorphe au quotient $G / g_{\alpha}$ de $G$ par le groupe de stabilité $g_{\alpha}$ de l'un de ses points; et toute mesure $\mu$ sur $E$ s'écrit d'une manière et d'une seule comme somme de mesures $\mu_{\alpha}$ respectivement portées par les orbites $\boldsymbol{E}_{\alpha}$.

On procédera par récurrence sur le nombre $N$ des orbites $E_{\alpha}$. Soient $a_{\alpha}$, pour $1 \leqslant \alpha \leqslant N$, des représentants de ces orbites. Soit $V$ un voisinage compact de $e$ dans $G$; par hypothèse, $G$ admet un recouvrement par une famille dénombrable d'ensembles de la forme $g_{i} V$; les ensembles $g_{i} V a_{\alpha}$ forment donc un recouvrement de $E$. Comme tout espace localement compact a la "propriété de Baire ", il s'ensuit que l'un au moins des $g_{i} V a_{\alpha}$ a un point intérieur; pour cette valeur de $\alpha$, l'orbite $E_{\alpha}=G a_{\alpha}$ est donc ouverte dans $E$, et à plus forte raison dans son adhérence dans $E$. Le même raisonnement montre alors que, si $V^{\prime}$ est un voisinage compact de $e$ dans $V, V^{\prime} a_{\alpha}$ a un point intérieur; donc $x \rightarrow x a_{\alpha}$ est une application ouverte de $G$ sur $E_{\alpha}$, et, si $g_{\alpha}^{\prime}$ est le groupe de stabilité de $a_{\alpha}, E_{\alpha}$ est isomorphe à $G / g_{\alpha}$. Pour obtenir la première partie du lemme, il n'y a qu'à appliquer l'hypothèse de récurrence à $E^{\prime}=E-E_{\alpha}$, qui est fermé dans $E$ et est réunion de $N-1$ orbites de $G$. Soient enfin $\mu$ une mesure sur $E, \mu_{\alpha}$ sa restriction à l'orbite ouverte $E_{\alpha}$, et $\mu^{\prime}=\mu-\mu_{\alpha} ;$ le support de $\mu^{\prime}$ étant contenu dans $E^{\prime}$, on peut lui appliquer l'hypothèse de récurrence, d'où la dernière conclusion.

Appliquant le lemme 16 à la mesure $\mu_{i}$ et aux orbites du groupe $G \times \operatorname{Aut}(X, i)$ dans $i_{X}^{-1}(\{i\})$, on voit qu'on peut écrire $\mu_{i}$ comme somme de mesures respectivement portées par ces orbites; il résulte alors de l'unicité de cette décomposition que chacune de ces dernières mesures satisfait, comme $\mu_{i}$, à la condition (25).

36. On va maintenant achever de déterminer $\mu_{i}$ en montrant qu'une seule de ces orbites, au plus, peut porter une telle mesure. Pour cela, introduisons encore une définition. Supposons d'abord $\mathcal{A}$ de type (I) ou (II); on conviendra de dire qu'un élément $x$ de $X$ est de rang quasimaximal s'il est de rang maximal, ou bien si $\mathcal{A}$ est de type $\left(\mathrm{I}_{0}\right)$, si $m=2 n-2$, si $i_{X}(x)=0$ et si $x$ est de rang $n-1$. En général, on dira qu'un élément $x$ de $X$ est de rang quasimaximal si, pour chacune des composantes simples de $\mathcal{A}$, la composante correspondante de $x$ est de rang quasimaximal. Pour tout $i \in I(X)$, on désignera par $\check{U}(i)$ l'ensemble des éléments de $i_{X}^{-1}(\{i\})$ qui sont de rang quasimaximal dans $X$. On observera que, si $\mathcal{A}$ est de type (I) et si un élément $x$ de $X$ est de rang maximal $n$ et satisfait à $i_{X}(x)=0$, on a $m \geqslant 2 n$; c'est là un cas particulier des résultats $d u n^{\circ} 23$. On en conclut immédiatement, d'abord dans les cas (I) et (II), puis dans le cas général, que tous les éléments de $\vec{U}(i)$ ont même rang pour $i$ donné; d'après le corollaire de la proposition 4 du Chapitre II, $\mathrm{n}^{\circ} \mathbf{2 5}$, $\bar{U}(i)$ est donc, soit vide, soit une orbite de $G \times \operatorname{Aut}(X, i)$. 
Lемме 17. Supposons que $\mathcal{A}$ et $X$ satisfassent à la condition $\left(\mathbf{A}^{\prime}\right) d u n^{\circ} 34 ;$ soit $i \in I(X)$, et soit $U$ une orbite de $G \times \operatorname{Aut}(X, i)$ dans $i_{X}^{-1}(\{i\})$ sur laquelle il existe une mesure $\mu \neq 0$ satisfaisant à (25). Alors $U=\bar{U}(i)$.

Soit $x_{0}$ un point de $U$, de sorte qu'on a $i=i_{X}\left(x_{0}\right)$; soient $n=\left(n_{v}\right)$ et $n^{\prime}=\left(n_{\nu}^{\prime}\right)$ les rangs de $X$ et de $x_{0}$, respectivement. Reprenant les notations du Chapitre II, $\mathrm{n}^{\circ} 25$, désignons par $N_{0}$ le noyau de $x_{0}$ dans $B$, par $B^{\prime}$ celui de $\lambda_{i}$, par $B^{n}$ un supplémentaire de $B^{\prime}$ dans $B$, et par $g^{\prime}$ le groupe des automorphismes de $B$ qui laissent $B^{\prime}$ et $B^{\prime \prime}$ invariants et induisent l'identité sur $B^{\prime \prime}$. Alors $N_{0}$ est un sous-module de $B^{\prime}$, et, d'après la proposition $4 \mathrm{du} \mathrm{n}^{\circ} 25$ et son corollaire, $U$ est l'orbite de $x_{0}$ par $G \times g^{\prime}$. Soit $G_{0}$ le groupe de stabilité de $x_{0}$ dans $G \times g^{\prime}$. Comme $G$ et $g^{\prime}$ sont unimodulaires, il en est de même de $G \times g^{\prime}$; d'après les théorèmes connus sur les mesures relativement invariantes dans les espaces homogènes (cf. [4], $\S 2$, $\mathrm{n}^{\circ} 6$, th. 3), toute mesure sur $U$, relativement invariante par $G \times g^{\prime}$, doit satisfaire, pour tout $(u, v) \in G_{0}$, à la formule

$$
d \mu\left(u x v^{-1}\right)=\left|\Delta_{G_{0}}(u, v)\right| d \mu(x)
$$

Soit $g^{\prime \prime}$ le groupe des éléments $v$ de $g^{\prime}$ tels que $(1, v) \in G_{0}$, ou autrement dit tels que $x_{0}=$ $x_{0} v$; si $B_{0}^{\prime}$ est un supplémentaire de $N_{0}$ dans $B^{\prime}$, on peut définir aussi $g^{\prime \prime}$ comme formé des automorphismes $v$ de $B$ qui, pour la décomposition $B=N_{0} \oplus B_{0}^{\prime} \oplus B^{\prime \prime}$ de $B$ en somme directe, s'écrivent sous forme matricielle comme suit :

$$
v=\left(\begin{array}{ccc}
v^{\prime \prime} & w & 0 \\
0 & 1 & 0 \\
0 & 0 & 1
\end{array}\right)
$$

avec $v^{\prime \prime} \in \operatorname{Aut}\left(N_{0}\right), w \in \operatorname{Hom}\left(B_{0}^{\prime}, N_{0}\right)$. Soit $G^{\prime \prime}$ le sous-groupe de $G_{0}$ formé des éléments de la forme $(1, v)$, avec $v \in g^{\prime \prime}$; pour $v$ donné par (27), désignons par $U^{\prime \prime}\left(v^{\prime \prime}, w\right)$ l'élément $(1, v)$ de $G^{\prime \prime}$.

Soit d'autre part $G^{\prime}$ le groupe des éléments $(u, v)$ de $G_{0}$ tels que $v$ induise l'identité sur $N_{0}$ et laisse $B_{0}^{\prime}$ invariant. Si $U_{0}=(u, v)$ appartient à $G_{0}$, on a $u x_{0} v^{-1}=x_{0}$, ce qui implique que $v$ laisse $N_{0}$ invariant; on voit aisément alors qu'on peut, d'une manière et d'une seule, déterminer $v_{1} \in g^{\prime \prime}$ de manière que $v \cdot v_{1}^{-1}$ induise l'identité sur $N_{0}$ et laisse $B_{0}^{\prime}$ invariant; il revient au même de dire que $U_{0}$ se met d'une manière et d'une seule sous la forme $U_{0}=$ $U^{\prime} U^{\prime \prime}$, avec $U^{\prime} \in G^{\prime}, U^{\prime \prime} \in G^{\prime \prime}$. On a donc $G_{0}=G^{\prime} \cdot G^{\prime \prime}$, et $G_{0}$ est le produit semidirect de $G^{\prime}$ et $G^{\prime \prime}$. Soient $d U^{\prime}$ une jauge invariante à gauche sur $G^{\prime}$, et $d v^{\prime \prime}, d w$ des jauges invariantes sur $\operatorname{Aut}\left(N_{0}\right)$ et sur le groupe additif $\operatorname{Hom}\left(B_{0}^{\prime}, N_{0}\right)$, respectivement. Si on écrit $U_{0}=U^{\prime} \cdot U^{\prime \prime}\left(v^{\prime \prime}, w\right)$ et qu'on pose

$$
\omega\left(U_{0}\right)=d U^{\prime} \wedge d v^{\prime \prime} \wedge d w
$$


on voit aussitôt que $\omega\left(U_{0}\right)$ est une jauge relativement invariante à gauche, donc aussi à droite, sur $G_{0}$, au moyen de laquelle on pourra calculer $\Delta_{G_{0}}$ comme il a été dit au $\mathrm{n}^{\circ} 4$ du Chapitre I.

Supposons maintenant qu'on ait $n_{v}^{\prime}<n_{\nu}$ pour une certaine valeur de $\nu$. Reprenons les notations des $\mathrm{n}^{\text {os }} 16,17$ et 25 ; désignons aussi par $N_{v}, B_{v}, B_{v}^{\prime}, B_{v}^{\prime \prime}$ les composantes relatives à $\mathfrak{f}_{v}$ de $N_{0}, B, B_{0}^{\prime}$ et $B^{\prime \prime}$, respectivement; ce sont des espaces vectoriels à droite sur $\mathfrak{f}_{v} ; B_{v}$ est somme directe de $N_{v}, B_{v}^{\prime}$ et $B_{v}^{\prime \prime} ; B_{v}$ est de dimension $n_{v}$, et $N_{v}$ de dimension $n_{v}-n_{v}^{\prime}$, sur $\mathfrak{I}_{v}$. Pour $t \in k, t \neq 0$, soit $v_{t}^{\prime \prime}$ l'automorphisme de $N_{0}$ qui induit $t \cdot 1$ sur $N_{v}$ et l'identité sur toutes les autres composantes de $N_{0}$; soit $v_{t}$ l'automorphisme de $B$ qui induit $v_{t}^{\prime \prime} \operatorname{sur} N_{0}$ et l'identité sur $B_{0}^{\prime}$ et sur $B^{\prime \prime} ;\left(1, v_{t}\right)$ appartient à $G^{\prime \prime}$. On va appliquer $(25)$ avec $u=1$, $v=v_{t}^{-1}$, et (26) avec $u=1, v=v_{t}$, et comparer les résultats. Comme $\left(1, v_{t}\right)$ est permutable avec tout élément de $G^{\prime}$ et que $d v^{\prime \prime}$ est invariante à droite et à gauche dans $\operatorname{Aut}\left(N_{0}\right)$, on a, avec les notations de (28) :

$$
\omega\left(\left(1, v_{t}\right)^{-1} \cdot U_{0} \cdot\left(1, v_{t}\right)\right)=d U^{\prime} \wedge d v^{\prime \prime} \wedge d\left(v_{t}^{-1} w\right) .
$$

Compte tenu des résultats du $\mathrm{n}^{\circ} 4 \mathrm{du}$ Chapitre $\mathrm{I}$, on aura donc, en posant $r=n_{v}-n_{\nu}^{\prime}$ et en désignant par $s$ la dimension de $B_{v}^{\prime}$ sur $\mathfrak{x}_{v}$ :

$$
\Delta_{G_{0}}\left(1, v_{t}\right)=t^{-\delta r s}
$$

où $\delta$ est la dimension de $\mathfrak{f}_{v}$ sur $k$.

Considérons d'abord le cas où $\mathcal{A}_{\nu}$ est de type $(\mathrm{I})$; reprenant nos notations habituelles relatives à ce type, écrivons $m, n$ au lieu de $m_{v}, n_{v}$, et $n^{\prime}$ au lieu de $n_{v}^{\prime}$ d'où $r=n-n^{\prime}$. Il est immédiat qu'on a :

$$
\left|v_{t}\right|_{X}=|t|^{\delta r m},\left|\bar{v}_{t}\right|_{I(X)}=|t|^{\delta r(n+2 \varepsilon-1)}
$$

La comparaison de (25) et de (26) donne alors

$$
m=n+2 \varepsilon-1+s .
$$

Comme $s \leqslant n^{\prime} \leqslant n-1$, on en conclut $m \leqslant 2 n+2 \varepsilon-2$, ce qui contredit l'hypothèse $\left(\mathbf{A}^{\prime}\right)$ si $\varepsilon \neq 0$. Si $\varepsilon=0,\left(\mathbf{A}^{\prime}\right)$ donne $m \geqslant 2 n-2$; on doit donc avoir $m=2 n-2, s=n^{\prime}=n-1, r=1$, de sorte que $N_{v} \oplus B_{v}^{\prime}$ est de dimension $n$ et coïncide donc avec $B_{v}$. Comme $N_{v} \oplus B_{v}^{\prime}$ est l'espace des vecteurs de $B_{v}$ orthogonaux à $B_{v}$ par rapport à la forme $\eta$-hermitienne déterminée par la composante $i_{\nu}$ de $i$ relative à $\mathcal{A}_{\nu}$, on en conclut que $i_{\nu}=0$, ce qui achève de montrer que la composante de $x_{0}$ relative à $\mathcal{A}_{\nu}$ est de rang quasimaximal au sens de la définition donnée plus haut.

Supposons enfin que l'involution $\iota$ transforme $\mathcal{A}_{v}$ en $\mathcal{A}_{v^{\prime}}$, avec $\boldsymbol{v}^{\prime} \neq v$, de sorte que $\mathcal{A}_{\nu} \oplus \mathcal{A}_{v^{\prime}}$ est de type (II); reprenant nos notations habituelles relatives à ce type, écrivons $m, p, q$ au lieu de $m_{v}, n_{v}, n_{v^{\prime}}$, et $p^{\prime}$ au lieu de $n_{v}^{\prime}$, d'où $r=p-p^{\prime}$. On a alors 


$$
\left|v_{t}\right|_{X}=|t|^{\delta r m}, \quad\left|\bar{v}_{t}\right|_{(X)}=|t|^{\delta r q} .
$$

La comparaison de (25) et de (26) donne ici $m=q+s$. Comme on a $s \leqslant p^{\prime} \leqslant p-1$, cela contredit $\left(\mathbf{A}^{\prime}\right)$, ce qui achève la démonstration.

37. Si (A) et $\left(\mathbf{A}^{\prime}\right)$ sont toutes deux satisfaites, il résulte du $\mathrm{n}^{\circ} 35$ et 'du lemme 17 que, pour tout $i \in I(X)$, la mesure $\mu_{i}$ est portée par l'orbite $\bar{U}(i)$. Les lemmes 9 du $\mathrm{n}^{\circ} 19$ et 10 du $\mathrm{n}^{\circ} 21$ montrent d'ailleurs que $i_{X}$ est submersive en tout point de $\bar{U}(i)$; et il est immédiat, d'abord dans les cas (I) et (II), puis en général, que $\bar{U}(i)$ est toujours une partie ouverte de $i_{X}^{-1}(\{i\})$. D'après le $\mathrm{n}^{\circ} 6 \mathrm{du}$ Chapitre $\mathrm{I}, \mu_{i}$ coinncide avec la mesure $\left|\theta_{i}\right|$ déterminée sur $\bar{U}(i)$ par la jauge

$$
\theta_{i}(x)=\left(\frac{d x}{d i_{X}(x)}\right)_{i}
$$

cette formule ayant le sens expliqué au $\mathrm{n}^{\circ} 6 \mathrm{du}$ Chapitre I; autrement dit, quel que soit $x_{0} \in \bar{U}(i), \theta_{i}(x)$ coīncide au voisinage de $x_{0}$ avec la forme induite sur $\bar{U}(i)$ par n'importe quelle forme différentielle $\eta(x)$ dans $X$ satisfaisant au voisinage de $x_{0}$ à la relation $d x=$ $\eta(x) \wedge d i_{X}(x)$.

On peut résumer comme suit les résultats ci-dessus :

Propostiton 6. Supposons que $\mathcal{A}$ et $X$ satisfassent à $(\mathbf{A})$ et à $\left(\mathbf{A}^{\prime}\right) ;$ soit $i \in I(X)$; soit $\bar{U}(i)$ l'ensemble des points de $i_{X}^{-1}(\{i\})$ de rang quasimaximal dans $X$, et soit $\theta_{i}$ la jauge sur $\bar{U}(i)$ définie par (29). Alors la mesure $\left|\theta_{i}\right|$ sur $\bar{U}(i)$ détermine une mesure tempérée dans $X$. De plus, pour tout $\Phi \in S(X)$, les fonctions $F_{\Phi}, F_{\Phi}^{*}$ respectivement définies sur $I(X)$ et sur $I(X)^{*}$ par les formules

$$
F_{\Phi}(i)=\int_{\bar{U}(i)} \Phi(x)\left|\theta_{i}(x)\right|, \quad F_{\Phi}^{*}\left(i^{*}\right)=\int_{X} \Phi(x) \chi\left(\left[i_{X}(x), i^{*}\right]\right)|d x|
$$

sont continues, intégrables, et sont les transformées de Fourier l'une de l'autre; en particulier, on $a \int \Phi|d x|=\int F_{\Phi}|d i|$.

En réalité, comme on l'a annoncé au $\mathbf{n}^{\circ} 34,(\mathbf{A})$ est équivalente à ( $\left.\mathbf{A}^{\prime}\right)$; mais, dans les chapitres suivants, il nous suffira de savoir que l'une et l'autre de ces conditions sont conséquences des conditions suffisantes énoncées dans la proposition 5 du $n^{\circ} 31$.

38. Lorsque les hypothèses de la proposition 6 sont vérifiées, celle-ci montre en particulier qu'on ne peut avoir $\mu_{i}=0$, pour $i$ donné dans $I(X)$, que si $\bar{U}(i)$ est vide; il est souvent important de savoir dans quels cas cette circonstance peut se présenter; à cet égard, nous nous contenterons d'énoncer les résultats suivants, qui en substance sont bien connus. 
Convenons d'abord de dire que $\mathcal{A}$ et $X$ satisfont à la condition $(\mathbf{B})$ si $m>2 n+4 \varepsilon-2$ dans le cas (I), si $m>p+q$ dans le cas (II), et, dans le cas général, si ces conditions sont satisfaites par chaque composante simple de $\mathcal{A}$ et par la composante correspondante de $X$; convenons aussi de dire que $\mathcal{A}$ et $X$ satisfont à la condition $\left(\mathbf{B}^{\prime}\right)$ si $m \geqslant 2 n+4 \varepsilon-2$ dans le cas (I); si $p=q$ et $m \geqslant p+q$ dans le cas (II), et; dans le cas général, si ces conditions sont satisfaites par chaque composante simple de $\mathcal{A}$ et par la composante correspondante de $X ;\left(\mathbf{B}^{\prime}\right)$ n'est autre que la condition suffisante de la proposition 5 du $\mathbf{n}^{\circ} 31$, qui, d'après cette proposition, entraîne (A), et qui entraîne évidemment aussi $\left(\mathbf{A}^{\prime}\right)$.

Cela posé, on a déjà fait observer au $\mathrm{n}^{\circ} 23 \mathrm{du}$ Chapitre II que, dañ le cas (II), la condition $m \geqslant p+q$, c'est-à-dire $\left(\mathbf{B}^{\prime}\right)$, est nécessaire et suffisante pour que $U(0)$ ne soit pas vide, et suffisante pour que $U(i)$ ne soit pas vide quel que soit $i \in I(X)$. Dans le cas $\left(I_{0}\right)$, on démontre élémentairement que $m \geqslant 2 n-2$, c'est-à-dire encore $\left(\mathbf{B}^{\prime}\right)$, est nécessaire et suffisant pour que $\tilde{U}(0)$ ne soit pas vide, et qu'alors $U(i)$ n'est pas vide quel que soit $i \neq 0$; il en serait ainsi même si on ne supposait pas que $k$ fût un corps local. Dans le cas (I) avec $\varepsilon \neq 0$, on sait, pour $n=1$ (cf. p. ex. [9]), et on vérifie aisément à partir de là par récurrence sur $n$, dans le cas général, que $U(i)$ n'est jamais vide quand la condition (B) est satisfaite, pourvu que $k$ soit un corps local à valuation discrète. Par suite, si $k$ est un corps local à valuation discrète, $(\mathbf{B})$ entraîne toujours $U(i) \neq \varnothing$ quel que soit $i \in I(X)$.

Rappelons enfin que, si $\mathcal{A}$ est de type (I) sur le corps local $k$ et que $X$ soit de rang $n=1$, la condition $U(0)=\varnothing$ équivaut à la compacité du groupe $G ; G$ n'est jamais compact dans le cas (II).

\section{La série d'Eisenstein-Siegel}

39. A partir de maintenant, on supposera une fois pour toutes que le corps de base $k$ est un corps de nombres algébriques, et on adoptera les hypothèses et notations de [14], Chapitres II et V, ainsi que celles des Chapitres I et II ci-dessus; en particulier, $\mathcal{A}_{k}$ désignera toujours une algèbre semisimple sur $k$, et $X_{k}$ un $\mathcal{A}_{k}$-module à gauche.

Soit $\Phi \in S\left(X_{A}\right)$; le groupe $M p(X / A)_{A}$ opère sur $S\left(X_{A}\right)$ comme il a été expliqué dans [14], Chapitres III et $V$, de sorte que $S \Phi$ est défini pour tout $S \in M p(X / \mathcal{A})_{A}$; en particulier, pour $s \in P s\left(X_{k} / \mathcal{A}_{k}\right), \mathbf{r}_{k}(s) \Phi$ est défini, et même est donné explicitement par la formule (38) de [14], Chapitre III, $n^{\circ}$ 40. Au moyen de cette formule, ou directement, on voit aussitôt que $\mathbf{r}_{k}(p) \Phi(0)=\Phi(0)$ quel que soit $p \in P\left(X_{k} / A_{k}\right)$; il s'ensuit que, pour $\Phi$ donné, $\mathbf{r}_{k}(s) \Phi(0)$ est constant sur les classes à gauche suivant $P\left(X_{k} / \mathcal{A}_{k}\right)$ dans $P s\left(X_{k} / \mathcal{A}_{k}\right)$. Si $R$ est un système de représentants de ces classes, la série

$$
E(\Phi)=\sum_{s \in R} \mathbf{r}_{k}(s) \Phi(0),
$$


qu'on appellera la série d'Eisenstein-Siegel relative à $\mathcal{A}_{k}$ et $X_{k}$, est donc indépendante du choix de $R$. Son étude formera l'objet principal de ce chapitre.

Il s'agira avant tout de donner une condition suffisante de convergence pour la série $E(\Phi)$. Observons d'abord que la question ne se pose pas quand $A$ est de type (II) et que $p=0$ ou $q=0$, puisqu'alors, d'après le $n^{0} 21, P\left(X_{k} / \mathcal{A}_{k}\right)$ coinncide avec $P_{s}\left(X_{k} / \mathcal{A}_{k}\right)$, de sorte que $E(\Phi)$ se réduit à $\Phi(0)$, ni quand $\mathcal{A}$ est de type $\left(\mathrm{I}_{0}\right)$ avec $n=1$, puisqu'alors, comme on l'a fait remarquer au n ${ }^{\circ} 29, P\left(X_{k} / \mathcal{A}_{k}\right)$ est d'indice 2 dans $P s\left(X_{k} / \mathcal{A}_{k}\right)$.

Pour aborder la question dans le cas général, on suivra une méthode analogue à celle qui a servi à traiter le "problème local " au $n^{\circ} 31$. Pour abréger, écrivons de nouveau $P s$, $P, Q$ au lieu de $P s(X / \mathcal{A}), P(X / \mathcal{A}), Q(X / \mathcal{A})$, de sorte que la série $E(\Phi)$ est étendue à $P_{k} \backslash P s_{k}$; $\pi$ dénotant cette fois la projection canonique de $M p(X / A)_{A}$ sur $P s_{A}$, on définira une fonction continue $f_{\Phi}$ sur $P s_{A}$ en posant, pour tout $\mathrm{S} \in M p(X / \mathcal{A})_{A}$ :

$$
f_{\Phi}(\pi(\mathbf{S}))=|\mathbf{S} \Phi(0)| \text {. }
$$

Pour tout élément $p=t(q) d(\lambda)$ de $P_{A}$, avec $q \in Q_{A}, \lambda \in \operatorname{Aut}(X)_{A}$, posons $\psi(p)=|\lambda| \frac{t}{x}$, où $|\lambda|_{X}$ désigne le module de l'automorphisme $\lambda$ de $X_{A}$. En raisonnant exactement comme on a fait au no 31 dans le cas local, on voit qu'on a $f_{\Phi}(p s)=\psi(p) f_{\Phi}(s)$ quels que soient $p \in P_{A}$ et $s \in P s_{A}$. Pour $\lambda \in \operatorname{Aut}(X)_{k}, \lambda$ laisse invariant $X_{k}$, qui est un sous-groupe discret de $X_{A}$ à quotient compact, de sorte que $|\lambda|_{x}=1$; on a donc $\psi(p)=1$ pour $p \in P_{k}$.

L Е Мм Е 18. Soit $f_{0}$ une fonction continue, partout $>0$ sur $P_{s_{A}}$, telle que $f_{0}(p s)=\psi(p) f_{0}(s)$ quels que soient $p \in P_{A}$ et $s \in P_{s_{A}}$. Alors, pour que la série $E(\Phi)$ soit absolument convergente quelle que soit $\Phi \in S\left(X_{A}\right)$, il faut et il suffit que la série $\sum_{R} f_{0}(s)$ soit convergente; et, quand il en est ainsi, $E(\Phi)$ est absolument convergente, uniformément par rapport à $\Phi$ sur toute partie compacte de $S\left(X_{A}\right)$.

Comme $P$ est un sous-groupe parabolique de $P s$, l'espace $P_{A} \backslash P_{s_{A}}$ des classes à gauche suivant $P_{A}$ dans $P_{s_{A}}$ est compact, et isomorphe à $(P \backslash P s)_{A}$; soit $C_{0}$ une partie compacte de $P s_{A}$ telle que $P s_{A}=P_{A} \cdot C_{0}$. En raisonnant alors comme on a fait dans le cas local à la fin $\mathrm{du} \mathrm{n}^{\circ} 30$, on voit immédiatement qu'on peut, à toute partie compacte $C$ de $S\left(X_{A}\right)$, faire correspondre $c>0$ tel que $f_{0}^{-1} f_{\Phi} \leqslant c$ sur $P_{s_{A}}$ quel que soit $\Phi \in C$; donc, si $\sum_{R} f_{0}(s)$ est convergente, $E(\Phi)$ est absolument convergente uniformément sur $C$. Quant à la réciproque, soit $\Phi$ telle que $\Phi(0) \neq 0$, donc $f_{\Phi}(e) \neq 0$; en vertu de la compacité de $C_{0}$, on peut choisir des $s_{i} \in P_{s_{A}}$ et des $c_{i}>0$, en nombre fini, tels que $f_{0}(s) \leqslant \sum c_{i} f_{\Phi}\left(s s_{i}\right)$ quel que soit $s \in C_{0}$, et par suite aussi quel que soit $s \in P_{s_{A}}$. Pour chaque $i$, soient $S_{i} \in M p(X / A)_{A}$ tel que $\pi\left(S_{i}\right)=s_{i}$, et $\Phi_{i}=S_{i} \Phi$; on aura $f_{\Phi}\left(s s_{i}\right)=f_{\Phi_{i}}(s)$. La convergence absolue des séries $E\left(\Phi_{i}\right)$ entraîne donc celle de $\sum_{R} f_{0}(s)$. 
Comme dans le cas local, il existe toujours des fonctions $f_{0}$ ayant les propriétés énoncées dans le lemme 18. On conclut immédiatement du lemme 18, en particulier, que la convergence absolue de $E(\Phi)$ quelle que soit $\Phi \in S\left(X_{A}\right)$, pour $A$ et $X$ donnés, équivaut à la conjonction des conditions analogues relatives à chacune des composantes simples de $\mathcal{A}$ et à la composante correspondante de $X$. Autrement dit, il suffit de discuter la convergence de $E(\Phi)$ quand $\mathcal{A}$ est de type (I) ou (II). De plus, au moyen de l'opération de " restriction du corps de base " (cf. [13], Chap. I), on voit que, dans l'un et l'autre de ces cas, on peut toujours supposer $z_{0}=k$; pour des raisons analogues, on fera presque toujours cette hypothèse dans toute la suite de ce travail.

40. On va maintenant établir le théorème suivant :

ThÉor ìme 1. Pour que la série $E(\Phi)$, définie par $(30)$ au $n^{\circ} 39$, soit absolument convergente quel que soit $\Phi \in S\left(X_{A}\right)$, et le soit uniformément sur toute partie compacte de $S\left(X_{A}\right)$, il suffit que $\mathcal{A}_{k}$ et $X_{k}$ satisfassent à la condition (B) $d u n^{\circ} 38$; en particulier, il suffit pour cela que $m>2 n+4 \varepsilon-2$ si $\mathcal{A}_{k}$ est de type (I), et que $m>p+q$ si $\mathcal{A}_{k}$ est de type (II).

D'après ce qui précède, on peut se borner à considérer les cas (I) et (II), en supposant $z_{0}=k$; on exclura de plus le cas trivial où $\mathcal{A}_{k}$ est de type (II) et où $p=0$ ou $q=0$, puisqu'alors $E(\Phi)$ se réduit à $\Phi(0)$, et aussi le cas $\left(\mathrm{I}_{0}\right)$ arec $n=1$, où $E(\Phi)$ ne comprend que deux termes. Dans tout autre cas, considérons à nouveau le sous-groupe $P s^{\prime}=P s^{\prime}(X / \mathcal{A})$ de $P s(X / \mathcal{A})$ qu'on a introduit au $n^{\circ} 28$; soit $P^{\prime}=P s^{\prime} \cap P$, et soit $R^{\prime}$ un système de représentants des classes à gauche suivant $P_{k}^{\prime}$ dans $P s_{k}^{\prime}$. Alors $P s^{\prime}$ est connexe et semisimple, $P^{\prime}$ est un sousgroupe parabolique de $P s^{\prime}$, et le lemme 11 du Chapitre II, $n^{\circ} 29$, montre qu'on peut prendre pour $R$ la réunion d'un nombre fini d'ensembles de la forme $R^{\prime} r$; il se trouve même que ce nombre est égal à 2 dans le cas $\left(\mathrm{I}_{0}\right)$ et à 1 dans tout autre cas, mais peu nous importe ici. Mais, si $r$ est donné dans $P s_{k}$, et si $f_{0}$ est la fonction considérée dans le lemme 18 du $\mathrm{n}^{\circ} 39, f_{0}(s)^{-1} f_{0}(s r)$ est bornée sur $P s_{A}$; donc, pour que la série $\sum_{R} f_{0}(s)$ soit convergente, il faut et il suffit que la série analogue restreinte à $R^{\prime}$ le soit.

On appliquera alors à cette série un critère de convergence dû à Godement (cf. [7]), d'après lequel il suffit, pour que la série en question soit convergente, que l'on ait, pour tout $p \in P_{A}^{\prime}$ :

$$
\psi(p)=\left|\Delta_{P^{\prime}}(p)\right|_{A}^{\tau}
$$

avec $\tau>1$; naturellement, $\psi$ est ici la fonction introduite au $\mathrm{n}^{\circ} 39$. Comme $P^{\prime}$ est un sousgroupe invariant de $P, \Delta_{P}$, coïncide avec $\Delta_{P}$ sur $P^{\prime}$ (cf. la remarque finale du $\mathrm{n}^{\circ} 5 \mathrm{du}$ Chapitre I), et $\Delta_{P}$ est donné par le lemme 12 du Chapitre $I I, \mathrm{n}^{\circ} 29$, c'est-à-dire par $\Delta_{P}(p)=$ $\Delta(\lambda)^{-1}$ pour $p=t(q) d(\lambda)$; de plus, on a alors $\psi(p)=|D(\lambda)|_{A}^{\mathbf{t}}$ si $D(\lambda)$ désigne, comme au $n^{\circ} 33$, 
le déterminant de $\lambda$ par rapport à une base de $X_{k}$ sur $k$. Dans le cas (I), le calcul de $D(\lambda)$, $\Delta(\lambda)$ a déjà été effectué au $n^{\circ} 33$ à l'occasion du problème local; il montre que $\psi$ est bien de la forme (31), avec $\tau=m /(2 n+4 \varepsilon-2)$, ce qui achève la démonstration dans ce cas. Dans le cas (II), on a un calcul tout à fait analogue, à partir des résultats du $\mathrm{n}^{\circ} 21$ du Chapitre II; comme il ne présente pas de difficulté, nous l'omettrons ici; on trouve que $D(\lambda)$ et $\Delta(\lambda)$ sont en général (ou, plus précisément, chaque fois que $p \neq q$ ) des caractères indépendants de $\operatorname{Aut}(X)$, mais que, sur $P^{\prime}$, on a de nouveau une relation de la forme (31), avec $\tau=m /(p+q)$, d'où le résultat annoncé.

Divers exemples donnent à penser que la condition suffisante du théorème 1 est aussi nécessaire, en excluant bien entendu le cas où $\mathcal{A}_{k}$ a une composante de type (II) avec $p q=0$. Il serait intéressant de trancher cette question dans le cas général.

41. Dans tout le reste de ce Chapitre, on supposera que $\mathcal{A}_{k}$ et $X_{k}$ satisfont à la condition (B) du $n^{\circ} 38$. On va expliciter $E(\Phi)$ en prenant pour système de représentants $R$ de $P_{k} \backslash P s_{k}$ celui qui est donné par la proposition 8 de [14], $\mathrm{n}^{\circ} 51$, et dont on a déjà fait usage dans le cas local au ${ }^{0} 32$ du Chapitre III. Autrement dit, $R$ se compose de tous les éléments de $P s_{k}$ de la forme $s=d^{\prime}\left(\gamma_{1}\right) t\left(q_{1}\right) \otimes e_{2}$ lorsqu'on choisit de toutes les manières possibles un sous-module $X_{1}$ de $X_{k}$ isomorphe à son dual $X_{1}^{*}$, puis, pour chaque $X_{1}$, un isomorphisme $\gamma_{1}$ de $X_{1}^{*}$ sur $X_{1}$ et un supplémentaire $X_{2}$ de $X_{1}$ dans $X_{k}$, et qu'on prend pour $q_{1}$ tous les éléments de $Q\left(X_{1} / \mathcal{A}_{k}\right)$. Pour un tel élément $s$, on peut calculer $\boldsymbol{r}_{k}(s) \Phi$ au moyen de la formule (38) de [14], $\mathrm{n}^{\circ} 40$, ou bien directement au moyen des formules de [14], $\mathrm{n}^{\circ} 13$. En écrivant $Z_{k}$ au lieu de $X_{1}$, on obtient

$$
\mathbf{r}_{k}(s) \Phi(0)=\int_{z_{\Lambda}} \Phi(z) \chi\left(q_{1}(z)\right)|d z|_{A} .
$$

Cela conduit à grouper dans la série $E(\Phi)$ tous les termes relatifs à un même sous-module $Z_{k}$ de $X_{k}$. On conviendra done d'écrire, chaque fois que $Z_{k}$ est un sous-module de $X_{k}$ isomorphe à son dual $Z_{k}^{*}$ :

$$
E_{Z}(\Phi)=\sum_{a \in Q Z} \int_{Z_{A}} \Phi(z) \chi(q(z))|d z|_{A}
$$

où on a posé $Q_{Z}=Q\left(Z_{k} / \mathcal{A}_{k}\right)$. On a donc :

$$
E(\Phi)=\sum_{z_{\boldsymbol{k}}} E_{Z}(\Phi),
$$

la sommation étant étendue à tous les sous-modules $Z_{k}$ de $X_{k}$ tels que $Z_{k}$ soit isomorphe à $Z_{k}^{*}$; et, d'après le théorème $1,(\mathbf{B})$ entraîne que les séries (32) et (33) sont absolument convergentes, uniformément en $\Phi$ sur toute partie compacte de $S\left(X_{A}\right)$. 
Pour étudier les séries $E_{Z}(\Phi)$, on peut évidemment se borner au cas où $X_{k}$ lui-même est isomorphe à son dual $X_{k}^{*}$, et où $Z_{k}=X_{k}$. Supposons donc qu'il en soit ainsi, et identifions $Q\left(X_{k} / \mathcal{A}_{k}\right)$ avec le dual $I\left(X_{k}\right)^{*}=I(X)_{k}^{*}$ de l'espace $I\left(X_{k}\right)=I(X)_{k}$ comme il a été dit au $\mathrm{n}^{\circ} 15$ du Chapitre II. Posons de plus, pour tout $i^{*} \in I(X)_{A}^{*}$ :

$$
F_{\Phi}^{*}\left(i^{*}\right)=\int_{X_{A}} \Phi(x) \chi\left(\left[i_{X}(x), i^{*}\right]\right)|d x|_{A} .
$$

On aura alors :

$$
E_{X}(\Phi)=\sum_{i * I(X)_{k}} F_{\Phi}^{*}\left(i^{*}\right)
$$

et la convergence absolue de cette série, uniformément en $\Phi$ sur toute partie compacte de $S\left(X_{A}\right)$, résulte de ce qui précède. Autrement dit, si on substitue $X_{A}, I(X)_{A}, I(X)_{k}$ et $i_{X}$ à $X, G, \Gamma$ et $f$, respectivement, dans la proposition $2 \mathrm{du} \mathbf{n}^{\circ} 2$, Chapitre $\mathrm{I}$, la condition ( $\left.\mathrm{B}_{1}\right)$ de la fin de ce $n^{\circ}$ est satisfaite. Comme, en vertu des résultats de [14], la condition ( $\left.B_{0}\right)$ l'est évidemment aussi, il s'ensuit que la condition (B) de la proposition 2 est satisfaite. On a donc, en vertu de cette proposition :

$$
E_{X}(\Phi)=\sum_{i \in I(X) k} F_{\Phi}(i)
$$

où $F_{\Phi}$ est la transformée de Fourier de $F_{\Phi}^{*}$; de plus, cette transformée de Fourier est donnée, pour tout $i \in I(X)_{A}$, par la formule

$$
F_{\Phi}(i)=\int \Phi(x) d \mu_{i}(x)
$$

où $\mu_{i}$ est une mesure positive tempérée sur $X_{A}$, de support contenu dans $i_{X}^{-1}(\{i\})$; et $F_{\Phi}$ et $F_{\Phi}^{*}$ sont des fonctions continues et intégrables, sur $I(X)_{A}$ et $I(X)_{A}^{*}$ respectivement. Enfin, la proposition $2 \mathrm{du} \mathrm{n}^{\circ} 2$ fait voir que le second membre de (34) est absolument convergent; comme les $\mu_{i}$ sont des mesures positives, on conclut de là, au moyen du lemme 5 de [14], $n^{\circ} 41$, que ce second membre converge uniformément sur toute partie compacte de $S\left(X_{A}\right)$. D'après le lemme $2 \mathrm{du} \mathrm{n}^{\circ} 2$, cela montre que $E_{X}$ est une mesure tempérée positive, donnée par

$$
E_{X}=\sum_{i \in I(X)_{k}} \mu_{i}
$$

Enfin on conclut de même au moyen de (33) que $E$ est une mesure tempérée positive, somme des mesures $E_{Z}$.

42. Supposons de nouveau, jusqu'à nouvel ordre; que $\mathcal{A}_{k}$ et $X_{k}$ satisfont à (B) et que $X_{k}$ est isomorphe à son dual $X_{k}^{*}$. D'après les résultats du $\mathrm{n}^{\circ} 26 \mathrm{du}$ Chapitre II, et en particulier d'après la formule (17) de ce $\mathrm{n}^{\circ}$, il s'ensuit que, pour tout corps $K \supset k, \mathcal{A}_{K}$ et $X_{K}$ ont ces mêmes propriétés, donc à plus forte raison qu'ils satisfont à la condition $\left(\mathrm{B}^{\prime}\right) \mathrm{du} \mathbf{n}^{\circ} 38 \mathrm{du}$ 
Chapitre III. Par suite, pour tout $v, \mathcal{A}_{v}$ et $X_{v}$ ont les propriétés (A) et (A') du Chapitre III, de sorte qu'on peut leur appliquer tous les résultats de ce Chapitre, et en particulier la proposition $6 \mathrm{du} \mathrm{n}^{\circ} 37$. Comme d'ailleurs (B) implique $m>2 n-2$ dans le cas $\left(\mathrm{I}_{0}\right)$, il n'y aura pas ici de distinction à faire entre les variétés désignées par $U(i)$ et $\bar{U}(i)$ au Chapitre III.

Prenons alors pour $\Phi$, dans les formules $d \mathrm{n}^{\circ} 41$, une fonction de la forme

$$
\Phi(x)=\prod_{v} \Phi_{v}\left(x_{v}\right) \quad\left(x=\left(x_{v}\right) \in X_{A}\right)
$$

où le produit est étendu à toutes les places $v$ de $k$, où $\Phi_{v}$ appartient à $S\left(X_{v}\right)$ quel que soit $v$, et où, pour presque tout $v, \Phi_{v}$ est la fonction caractéristique de $X_{v}^{\circ}$. La fonction $\Phi$ étant ainsi choisie, désignons par $F_{v}$ et $F_{v}^{*}$, pour tout $v$, les fonctions respectivement définies sur $I(X)_{v}$ et sur $I(X)_{v}^{*}$ par les formules

$$
F_{v}(i)=\int_{U_{v}(i)} \Phi_{v}(x)\left|\theta_{i}(x)\right|_{v}, \quad F_{v}^{*}\left(i^{*}\right)=\int_{X_{v}} \Phi_{v}(x) \chi_{v}\left(\left[i_{X}(x), i^{*}\right]\right)|d x|_{v}
$$

ici on a noté $U_{v}(i)$ la variété formée des points de rang maximal de $i_{X}^{-1}(\{i\})$ dans $X_{v}$, et $\theta_{i}$ la jauge définie sur cette variété par la formule (29) du $\mathrm{n}^{\circ} 37$. D'après la proposition 6 du $\mathbf{n}^{\circ} 37, F_{v}$ et $F_{v}^{*}$ sont continues, intégrables, et transformées de Fourier l'une de l'autre. D'après l'hypothèse faite sur $\Phi$, on voit immédiatement que $F_{v}^{*}$ prend la valeur constante 1 sur $\left(I(X)^{*}\right)_{v}^{\circ}$ pour presque tout $v$; ici, conformément à nos notations générales (cf. Chapitre $\left.I, \mathbf{n}^{\circ} 3\right),\left(I(X)^{*}\right)_{v}^{\circ}$ désigne le réseau dans $I(X)_{v}^{\circ}$ engendré par une base $\left(I(X)^{*}\right)^{\circ}$ arbitrairement choisie de $I(X)_{k}^{*}$ sur $k$.

Il est immédiat alors qu'on a, quel que soit $i^{*}=\left(i_{v}^{*}\right) \in I(X)_{A}^{*}$ :

$$
F_{\Phi}^{*}\left(i^{*}\right)=\prod_{v} F_{v}^{*}\left(i_{v}^{*}\right)
$$

pour tout $i^{*}$, d'après ce qui précède, presque tous les facteurs du second membre ont la valeur 1. On en déduit

$$
\int\left|F_{\mathbf{\Phi}}^{*}\left(i^{*}\right)\right| \cdot\left|d i^{*}\right|_{A}=\prod_{v} \int\left|F_{v}^{*}\left(i^{*}\right)\right| \cdot\left|d i^{*}\right|_{v}
$$

Dans cette relation, le premier membre est $<+\infty$; il est $\neq 0$ sauf si $F_{\Phi}^{*}=0$; d'ailleurs, on peut toujours modifier un nombre fini des fonctions $\Phi_{v}$ de manière à avoir $F_{\Phi}^{*} \neq 0$, par exemple en prenant $\Phi_{v} \geqslant 0$ et $\Phi_{v} \neq 0$ quel que soit $v$, ce qui entraîne $F_{\Phi} \neq 0$ et par suite $F_{\Phi}^{*} \neq 0$. Comme presque tous les facteurs du second membre sont $\geqslant 1$ d'après ce qui précède, il s'ensuit que ce second membre est absolument convergent (au sens défini dans la note $\left({ }^{1}\right.$ ) de la page 11). On en conclut aisément que la transformée de Fourier $F_{\Phi}$ de $F_{\Phi}^{*}$ est 
le produit des transformées de Fourier $F_{v}$ des $F_{v}^{*}$, c'est-à-dire qu'on a, quel que soit $i=\left(i_{v}\right) \in I(X)_{A}$ :

$$
F_{\Phi}(i)=\prod_{v} F_{v}\left(i_{v}\right)
$$

le produit du second membre étant absolument convergent.

Si on désigne par $\mu_{v}$ la mesure tempérée sur $I(X)_{v}$ déterminée par la mesure $\left|\theta_{i_{v}}\right|_{v}$ sur $U_{v}\left(i_{v}\right), F_{v}\left(i_{v}\right)$ n'est autre que $\mu_{v}\left(X_{v}^{\circ}\right)$ chaque fois que $\Phi_{v}$ est la fonction caractéristique de $X_{v}^{\circ}$. La formule ci-dessus montre donc que le produit des $\mu_{v}\left(X_{v}^{\circ}\right)$ est absolument convergent, et que la mesure $\mu_{i}$ qui figure dans l'expression (35) de $F_{\Phi}$ n'est pas autre chose que $\prod \mu_{v}$.

43. Quand $i$ appartient à $I(X)_{k}$, l'ensemble $i_{X}^{-1}(\{i\})$, sur le domaine universel, est une partie $k$-fermée de $X$. On désignera par $U(i)$ l'ensemble des points de rang maximal de cet ensemble; c'est une partie $k$-ouverte de $i_{X}^{-1}(\{i\})$; d'après la proposition 3 du Chapitre II, $\mathrm{n}^{\circ} 22$, quand $U(i)$ n'est pas vide, c'est une orbite du groupe $G$, pris lui aussi sur le domaine universel. On conclut facilement du lemme 8 du Chapitre II, $\mathbf{n}^{\circ} 17$, que, si $K \supset k$, l'ensemble $U(i)_{K}$ des points de $U(i)$ qui sont rationnels sur $K$ n'est autre que l'ensemble des points de $i_{X}^{-1}(\{i\})$ dans $X_{K}$ qui sont de rang maximal dans $X_{K}$. En particulier, pour $K=k_{v}$, on voit que $U(i)_{v}$ est l'ensemble qui a été désigné par $U_{v}(i)$ au $\mathrm{n}^{\circ} 42$.

Lемме 19. Pour tout $i \in I(X)_{k}$, l est un système de facteurs de convergence pour $U(i)$, et on $a \mu_{i}=\left|\theta_{i}\right|_{A}$.

Si $\Phi$ est choisi comme au $n^{\circ} 42$, on $a$, d'après ce qui précède :

$$
\int_{X_{A}} \Phi d \mu_{i}=\prod_{v} \int_{U(i)_{v}} \Phi_{v}\left|\theta_{i}\right|_{v}
$$

Soit $\lambda=\left(\lambda_{v}\right)$ un système de facteurs de convergence pour $U(i)$. Comme l'injection canonique de $U(i)$ dans $X$ est un morphisme, on a, pour presque tout $v, U(i)_{v}^{\circ} \subset X_{v}^{\circ}$. Si on suppose que $\Phi_{v} \geqslant 0$ pour tout $v$, on aura, d'après les définitions du $\mathrm{n}^{\circ} 4 \mathrm{du}$ Chapitre I :

$$
\int_{U(i)_{A}} \Phi \cdot\left|\lambda \theta_{i}\right|_{A}=\prod_{v} \lambda_{v} \int_{U(i)_{v}} \Phi_{v} \cdot\left|\theta_{i}\right|_{v}
$$

La comparaison de ces formules montre bien que, si on a le droit de prendre $\lambda=1, \Phi$ induit sur $U(i)_{A}$ une fonction intégrable pour $\left|\theta_{i}\right|_{A}$, et qu'on a alors $\mu_{i}=\left|\theta_{i}\right|_{A}$.

Il reste à démontrer la première assertion du lemme; il suffit évidemment pour cela de considérer le cas où $\mathcal{A}_{k}$ est de type (I) ou (II) avec $z_{0}=k$. Si $U(i)$ contient un point $x_{0}$ rationnel sur $k$, soit $g_{0}$ le groupe de stabilité de $x_{0}$ dans $G$. Alors $U(i)$ est isomorphe à 
$G / g_{0}$, et tout revient (d'après [13], théorèmes 2.4.2 et 2.4.3) à vérifier qu'il y a un système de facteurs de convergence commun à $G$ et à $g_{0}$; or c'est là une conséquence immédiate des résultats du $\mathrm{n}^{\circ} 23$ du Chapitre II, joints aux résultats connus sur les groupes classiques (cf. [13]).

Supposons que $U(i)_{k}$ soit vide; d'après les remarques du $\mathrm{n}^{\circ} 38 \mathrm{du}$ Chapitre III, cela implique que $\mathcal{A}_{k}$ n'est ni de type (II), ni de type $\left(\mathrm{I}_{0}\right)$. En vertu du "principe de Hasse " (dont il sera question au $\mathrm{n}^{\circ} 53$ du Chapitre VI), cela implique même que $\dot{U}_{(i)_{A}}$ est vide; mais, comme nous ne désirons pas faire usage de ce principe ici, nous ramènerons comme suit ce cas au précédent. D'après l'hypothèse $(\mathbf{B})$, on a $m \geqslant 2 n$; en désignant par $A$, comme au Chapitre II, l'espace $M_{m, 1}(\mathfrak{l})$, il est aisé de déduire de là qu'on peut définir sur $A \times A$ une forme $\eta$-hermitienne non dégénérée $h_{1}$ telle que $h_{1}\left[x_{1}\right]=i$ ait une solution $x_{1}$ de rang maximal dans $X_{k}$, ou autrement dit que $U_{1}(i)_{k}$ ne soit pas vide, $U_{1}(i)$ étant défini à partir de $h_{1}$ comme $U(i)$ l'est à partir de $h$. Soit $S$ un ensemble de places de $k$, comprenant en particulier toutes les places à l'infini, toutes les places "paires " (celles pour lesquelles $\mathfrak{p}_{v}$ divise 2 ) et les places $v$, en nombre fini d'après le $\mathrm{n}^{\circ} 4$ du Chapitre $\mathrm{I}$, pour lesquelles $U(i)_{v}^{\circ}$ ou $U_{1}(i)_{v}^{\circ}$ est vide; pour $v \notin S$, soient $N_{v}, N_{v}^{1}$ les nombres de points de $U(i)_{v}^{\circ}$ et de $U_{1}(i)_{v}^{\circ}$, respectivement, modulo $\mathfrak{p}_{v}$; nous sommes ramenés à démontrer que $\Pi\left(N_{v} / N_{v}^{1}\right)$ est absolument convergent, puisqu'alors, d'après les résultats rappelés au $n^{\circ} 4$ du Chapitre $I$, tout système de facteurs de convergence pour $U_{1}(i)$ en sera un aussi pour $U(i)$. On vérifie alors facilement que, pour presque tout $v, N_{v}$ est le nombre d'éléments d'un ensemble non vide, qui est un espace homogène analogue à $U(i)_{k}$ sur le corps fini $\mathfrak{o}_{k} / \mathfrak{p}_{k}$; par suite, c'est le quotient du nombre d'éléments d'un groupe analogue à $G$ par celui d'un groupe analogue à $g_{0}$; il en est de même, bien entendu, de $N_{v}^{1}$. Les formules connues sur le nombre d'éléments des groupes classiques sur les corps finis montrent alors immédiatement que $N_{v}=N_{v}^{1}$ pour presque tout $v$ si $A_{k}$ est de type $\left(\mathrm{I}_{1}\right)$ ou $\left(\mathrm{I}_{2}\right)$; elles montrent que $N_{v} / N_{v}^{1}=1+O\left(N p_{v}^{-2}\right)$ si $\mathcal{A}_{k}$ est de type $\left(\mathrm{I}_{4}\right)$, et aussi si $\mathcal{A}_{k}$ est de type $\left(\mathrm{I}_{3}\right)$ puisqu'alors $\mathcal{A}_{v}$ est de type $\left(\mathrm{I}_{4}\right)$ pour presque tout $v$. On pourra observer que le développement complet des indications qui précèdent contient la démonstration des résultats rappelés plus haut sur les systèmes de facteurs de convergence des groupes classiques et rendrait donc inutile la distinction des deux cas ci-dessus et l'introduction de l'orbite $U_{1}(i)$.

44. Nous pouvons maintenant résumer comme suit les résultats obtenus dans ce chapitre.

THÉoR ÈM 2. Supposons que $A_{k}$ et $X_{k}$ satisfassent à la condition (B), et que $X_{k}$ soit isomorphe à son dual $X_{k}^{*}$. Posons, pour $\Phi \in S\left(X_{A}\right)$ : 


$$
E_{X}(\Phi)=\sum_{Q \in Q_{X}} \int_{X_{A}} \Phi(x) \chi(q(x))|d x|_{A}
$$

où la sommation est étendue à $Q_{X}=Q\left(X_{k} / \mathcal{A}_{k}\right)$. Alors la série du second membre est absolument convergente, et $E_{X}$ est une mesure tempérée positive. De plus, pour tout $i \in I(X)_{k}, 1$ est un système de facteurs de convergence pour la variété $U(i)$ des points de $i_{\bar{X}}^{-1}(\{i\})$ de rang maximal; et, si $\theta_{i}$ désigne la jauge sur cette variété définie par la formule

$$
\theta_{i}(x)=\left(\frac{d x}{d i_{X}(x)}\right)_{i},
$$

la mesure $\left|\theta_{i}\right|_{A}$ sur $U(i)_{A}$ définit une mesure tempérée positive $\mu_{i}$ dans $X_{A}$, et on a

$$
E_{X}=\sum_{i \in i(X) k} \mu_{i} .
$$

Thtorème 3. Supposons que $\mathcal{A}_{k}$ et $X_{k}$ satisfassent à la condition (B). Posons, pour $\Phi \in \mathcal{S}\left(X_{A}\right)$ :

$$
E(\Phi)=\sum_{s \in P_{k} \backslash P s_{k}} \mathbf{r}_{k}(s) \Phi(0)
$$

où $P s_{k}=P s\left(X_{k} / \mathcal{A}_{k}\right), P_{k}=P\left(X_{k} / \mathcal{A}_{k}\right)$. Alors la série du second membre est absolument convergente; $E$ est une mesure tempérée positive; et on $a$ :

$$
E=\sum_{Z_{k}} E_{Z}
$$

où la sommation est étendue à tous les sous-modules $Z_{k}$ de $X_{k}$ tels que chaque $Z_{k}$ soit isomorphe à son dual $Z_{k}^{*}$, et où $E_{Z}$, pour tout $Z_{k}$, est défini par le théorème 2.

Formellement, le théorème 3 reste vrai, mais devient trivial, dans le cas où $\mathcal{A}_{k}$ est de type (II) et où $p=0$ ou $q=0$, même si (B) n'est pas satisfaite; $E(\Phi)$ se réduit alors à $\Phi(0)$, et le seul sous-module de $X_{k}$ isomorphe à son dual est $Z_{k}=\{0\}$. En général, on notera $E_{0}$, au lieu de $E_{\{0\}}$, la mesure $E_{Z}$ correspondant à $Z_{k}=\{0\}$; elle n'est autre évidemment que la mesure de Dirac $\delta_{0}$ relative au point 0 .

Si $\mathcal{A}_{k}$ est de type (I) et $X_{k}$ de rang 1 , ou si $\mathcal{A}_{k}$ est de type (II) et $X_{k}$ de rang $(1,1)$, on a $E=E_{0}+E_{X}$ puisqu'alors il n'y a pas de sous-module de $X_{k}$, isomorphe à son dual, autre que $\{0\}$ et $X_{k}$. En particulier, si $\mathcal{A}_{k}$ est de type $\left(\mathrm{I}_{0}\right)$ et $X_{k}$ de rang 1 , on a déjà observé au $n^{\circ} 39$ que la série d'Eisenstein-Siegel qui définit $E$ se réduit à deux termes; d'ailleurs, comme on a alors $Q_{X}=\{0\}$, on a $E_{X}(\Phi)=\int \Phi|d x|_{A}$; la plupart des résultats ci-dessus sont triviaux dans ce cas.

45. Les mesures introduites ci-dessus possèdent d'importantes propriétés d'invariance qu'on va maintenant énoncer. 
Pour cela, on transposera par dualité, à l'espace des distributions tempérées sur $X_{A}$, l'opération du groupe métaplectique $M p(X / \mathcal{A})_{A}$ sur $S\left(X_{A}\right)$; autrement dit, pour tout élément $\mathrm{S}$ de ce groupe, et toute distribution tempérée $T$ sur $X_{A}$, on définira $T^{\mathrm{S}}$ au moyen de $T^{\mathrm{S}}(\Phi)=T(\mathrm{~S} \Phi)$ pour tout $\Phi \in S\left(X_{A}\right)$. La définition de la série d'Eisenstein-Siegel $E(\Phi)$ montre alors que $E$ est invariante par $\mathbf{r}_{k}(s)$ pour tout $s \in P s\left(X_{k} / \mathcal{A}_{k}\right)$, ce qu'on exprimera plus brièvement en disant que $E$ est invariante par $P s\left(X_{k} / \mathcal{A}_{k}\right)$. En vertu de la proposition 8 de [14], $\mathrm{n}^{\circ}$ 51, il revient au même de dire que $E$ est invariante par les opérateurs suivants :

(a) $\mathbf{t}(q)$, pour tout $q \in Q\left(X_{k} / \mathcal{A}_{k}\right)$; autrement dit, on a $E(\Phi)=E\left(\Phi^{\prime}\right)$ chaque fois que $\Phi^{\prime}$ est défini par $\Phi^{\prime}(x)=\Phi(x) \chi(q(x))$, avec $q \in Q\left(X_{k} / A_{k}\right)$;

(b) $\mathrm{d}(\lambda)$, pour tout $\lambda \in \operatorname{Aut}\left(X_{k}\right)$; autrement dit, $E$ est invariante par l'automorphisme $x \rightarrow x \lambda$ de $X_{A}$ pour tout $\lambda \in \operatorname{Aut}\left(X_{k} / \mathcal{A}_{k}\right)$

(c) $d^{\prime}\left(\gamma_{1}\right) \otimes e_{2}$ chaque fois qu'on a écrit $X_{k}$ comme somme directe de deux sous-modules $X_{1}, X_{2}$ dont le premier est isomorphe à son dual, et que $\gamma_{1}$ est un isomorphisme de $X_{1}^{*}$ sur $X_{1}$; autrement dit, chaque fois qu'il en est ainsi et qu'on a identifié $X_{1}^{*} \oplus X_{2}$ avec $X_{1} \oplus X_{2}$ au moyen de $\gamma_{1}, E$ est invariante par la " transformation de Fourier partielle " relative $\grave{a}$ $\left(X_{1}\right)_{A}$.

Quand $X_{k}$ est isomorphe à son dual, (c) entraîne en particulier la condition suivante :

(c') $E$ est invariante par la transformation de Fourier lorsqu'on identifie $X_{A}^{*}$ avec $X_{A}$ au moyen d'un isomorphisme $\gamma$ de $X_{k}^{*}$ sur $X_{k}$.

Réciproquement, (c'), jointe à (a) et (b), entraîne (c) chaque fois que $P s\left(X_{k} / \mathcal{A}_{k}\right)$ est engendré par $d^{\prime}(\gamma)$ et $P\left(X_{k} / \mathcal{A}_{k}\right)$. Il en est ainsi par exemple chaque fois que $X_{k}$ est isomorphe à son dual et que $\mathcal{A}_{k}$ n'a pas de composante de type $\left(\mathrm{I}_{0}\right)$, comme on le voit en répétant la démonstration du corollaire 3 de la proposition 6 , [14], $\mathrm{n}^{\circ} 47$.

D'autre part, le corollaire de la proposition 9, [14], $\mathrm{n}^{\circ} 51$, montre immédiatement que $E$ possède aussi la propriété suivante :

(d) E est invariante par l'automorphisme $x \rightarrow u x$ de $X_{A}$ quel que soit $u \in G_{A}$, ou autrement $\operatorname{dit}$ pour tout $u \in \mathcal{A}_{A}$ tel que $u \cdot u^{\imath}=1$.

En fait, ce corollaire montre que chaque terme de la série qui définit $E$ possède cette propriété; il en est donc de même des mesures $E_{Z}$, puisque celles-ci ont été introduites au $n^{0} 41$ comme des séries partielles de la série d'Eisenstein-Siegel. Comme chacune des mesures $E_{Z}$, d'après le théorème 2 , a son support contenu dans la réunion des ensembles $i_{X}^{-1}(\{i\})$ pour $i \in I(X)_{k}$, et qu'on a $\chi(q(x))=1$ sur chacun de ces ensembles pour $q \in Q\left(X_{k} / A_{k}\right)$, les $E_{Z}$ ont aussi la propriété (a). Il est évident aussi que, si un automorphisme $\lambda$ de $X_{k}$ transforme $Z_{k}$ en $Z_{k}^{\prime}$, il transforme $E_{Z}$ en $E_{Z}$; en particulier, pour tout $Z_{k}, E_{Z}$ est invariant $\operatorname{par} \operatorname{Aut}\left(Z_{k} / \mathcal{A}_{k}\right)$. 


\section{Théorèmes d'unicité}

46. Lorsque $\mathcal{A}_{k}$ est de type (I), on peut caractériser par des propriétés intrinsèques la mesure $E$ et les mesures $E_{X}$ qu'on a étudiées au Chapitre IV; c'est là l'objet du présent Chapitre. La méthode qu'on va suivre s'étend sans difficulté au cas où $\mathcal{A}_{k}$ est de type (II) et $X_{k}$ de rang $(1,1)$, à condition de remplacer partout le groupe $P s(X / \mathcal{A})$ par le sous-groupe $P s^{\prime}(X / A)$ qui a été défini au Chapitre II, $\mathrm{n}^{\circ} 28$. Sans doute pourrait-elle même s'étendre au cas où $\mathcal{A}_{k}$ est de type (II) et $X_{k}$ de rang $(p, p)$ avec $p>1$, mais on aurait besoin pour cela d'une modification du lemme $6 \mathrm{du} \mathrm{n}^{\circ} 13$ dont je ne sais si elle est valable. Le cas (II), qui d'ailleurs ne semble pas pour l'instant se prêter à des applications arithmétiques intéressantes, sera complètement laissé de côté dans ce qui suit.

Dans ce Chapitre, nous supposerons done une fois pour toutes que $\mathcal{A}_{k}$ est de type (I) et que $A_{k}$ et $X_{k}$ satisfont à la condition (B), c'est-à-dire à $m>2 n+4 \varepsilon-2$; on supposera aussi $z_{0}=k$. Comme précédemment, on écrira le plus souvent $P s$ au lieu de $P s(X / \mathcal{A})$, donc $P s_{k}$ au lieu de $P s\left(X_{k} / \mathcal{A}_{k}\right)$ et $P s_{A}$ au lieu de $P s(X / \mathcal{A})_{A}$, et de même $M p_{A}$ au lieu de $M p(X / \mathcal{A})_{A}$. Nous identifierons $P s_{k}$ avec son image dans $M p_{A}$ au moyen de $\mathbf{r}_{k}$ chaque fois que cela ne risquera pas d'entraîner de confusion; comme au $\mathrm{n}^{\circ} 45$, cela permet de dire d'une mesure tempérée sur $X_{A}$ qu'elle est invariante par $P s_{k}$ quand elle l'est par $\mathbf{r}_{k}(s)$ quel que soit $s \in P s_{k}$ au sens qui a été expliqué au $n^{\circ} 45$. D'autre part, comme au $n^{\circ} 45$, on dira qu'une mesure (tempérée ou non) dans $X_{A}$ est invariante par un élément $u$ de $G_{A}$ si elle l'est par l'application $x \rightarrow u x$ de $X_{A}$ sur lui-même. Rappelons que, d'après le corollaire de la proposition 9 , [14], $\mathrm{n}^{\circ} 51$, les automorphismes $\Phi \rightarrow \mathbf{S} \Phi$ et $\Phi(x) \rightarrow \Phi(u x)$ de $S\left(X_{A}\right)$, pour $\mathbf{S} \in M p_{A}$ et $u \in G_{A}$, sont permutables; il en est donc de même des automorphismes correspondants de l'espace des distributions tempérées sur $X_{A}$.

Soit $\hat{E}$ une mesure tempérée sur $X_{A}$, invariante par $P s_{k}$, et soit $\Phi \in S\left(X_{A}\right)$; alors $\mathrm{S} \rightarrow \hat{E}(\mathrm{~S} \Phi)$ est une fonction continue sur $M p_{A}$, invariante à gauche par $P s_{k}$. On va commencer par donner des conditions pour que cette fonction soit bornée sur $M p_{A}$, uniformément en $\Phi$ sur toute partie compacte de $S\left(X_{A}\right)$; pour cela, on appliquera au groupe $P s$ les résultats de la "théorie de la réduction " qu'on a rappelés aux $n^{\text {os }} 9$ et 10 du Chapitre $I$.

Comme aux $n^{\text {os }} 18-19$ du Chapitre II, identifions $X_{k}$ avec $M_{m, n}(\mathfrak{f})$, et par suite Aut $\left(X_{k}\right)$ avec $G L(n, \mathfrak{f})$. Pour $x \in M_{m, n}(\mathfrak{f})$, on désignera par $x_{1}, \ldots, x_{n}$ les colonnes de la matrice $x$, de sorte qu'on a $x_{\alpha} \in M_{m, 1}(\mathfrak{l})$ pour $1 \leqslant \alpha \leqslant n$, et on écrira $x=\left(x_{1}, \ldots, x_{n}\right)$. Soit $t=\left(t_{1}, \ldots, t_{n}\right)$ un élément de $\left(G_{m}\right)^{n}$, où $G_{m}$ désigne comme d'habitude le groupe multiplicatif à une variable; on désignera par $\lambda_{t}$ l'automorphisme de $X$ défini par la matrice diagonale dont les éléments diagonaux sont $t_{1}, \ldots, t_{n}$; il s'écrit aussi

$$
x=\left(x_{1}, \ldots, x_{n}\right) \rightarrow x \lambda_{t}=\left(x_{1} t_{1}, \ldots, x_{n} t_{n}\right) .
$$

5-652922. Acta mathematica. 113. Imprimé le 1 mars 1965. 
Comme au $\mathrm{n}^{\circ} 19$ du Chapitre II, identifions $I(X)_{k}$ avec l'espace des matrices $\eta$-hermitiennes $i=\left(i_{\alpha A}\right)_{1 \leqslant \alpha, R \leqslant n}$ sur $\mathfrak{l}$. Pour $t \in\left(G_{m}\right)^{n}$, on notera $\bar{\lambda}_{t}$ l'automorphisme de $I(X)$ déterminé par l'automorphisme $\lambda_{t}$ de $X$; il s'écrit aussi

$$
i=\left(i_{\alpha \beta}\right) \rightarrow i \bar{\lambda}_{t}=\left(i_{\alpha \beta} t_{\alpha} t_{\beta}\right)
$$

Si $\delta$ désigne comme toujours la dimension de $\mathfrak{f u r} k$, les déterminants de $\lambda_{t}$ et de $\bar{\lambda}_{t}$, par rapport à des bases de $X_{k}$ et de $I(X)_{k}$ sur $k$, seront respectivement

$$
D\left(\lambda_{t}\right)=\left(t_{1} \ldots t_{n}\right)^{m \delta}, \quad D\left(\bar{\lambda}_{t}\right)=\left(t_{1} \ldots t_{n}\right)^{(n+2 \varepsilon-1) \delta} .
$$

On en conclut que la jauge $\theta_{i}(x)$ sur $U(i)$, définie dans le théorème 2 du Chapitre $I V, \mathrm{n}^{\circ} 44$, est transformée par $\lambda_{t}$ en la jauge

$\operatorname{sur} U\left(i^{\prime}\right)$, avec $i^{\prime}=i \vec{\lambda}_{i}$.

$$
\theta_{i}\left(x \lambda_{t}^{-1}\right)=\left(t_{1} \ldots t_{n}\right)^{(-m+n+2 \varepsilon-1) \delta} \theta_{i^{\prime}}(x)
$$

En particulier, pour $t \in\left(I_{k}\right)^{n}, \lambda_{t}$ et $\bar{\lambda}_{t}$ seront des automorphismes de $X_{A}$ et de $I(X)_{A}$, respectivement. Si on pose, pour abréger, $|t|_{A}=\left|t_{1} \ldots t_{n}\right|_{A}$, le module de $\lambda_{t}$ dans $X_{A}$ sera $\left|\lambda_{t}\right|_{X}=|t|_{A}^{m \delta}$, de sorte qu'on aura, pour $\Phi \in S\left(X_{A}\right), x=\left(x_{1}, \ldots, x_{n}\right):$

$$
\mathbf{d}\left(\lambda_{t}\right) \Phi(x)=|t|_{A}^{m \delta / 2} \Phi\left(x_{1} t_{1}, \ldots, x_{n} t_{n}\right)
$$

47. Comme on l'a vu au $\mathrm{n}^{\circ} 28 \mathrm{du}$ Chapitre II, $P s$ est un groupe algébrique réductif. On désignera par $T$ l'image de $\left(G_{m}\right)^{n}$ dans $P s$ par $t \rightarrow d\left(\lambda_{t}\right)$; il est bien connu que $T$ est un tore trivial maximal de $P s$. La détermination des racines de $T$ dans $P s$ se fait sans difficulté; on trouve qu'on peut les ordonner de manière que les racines strictement positives (c'està-dire $\neq 1$ et $>1$ ) soient les $t_{\alpha} t_{\beta}^{-1}$ et les $t_{\alpha} t_{\beta}$ pour $1 \leqslant \alpha<\beta \leqslant n$, ainsi que les $t_{\alpha}^{2}$ pour $1 \leqslant \alpha \leqslant n$ dans le cas où $\varepsilon>0$. Soit $T_{A}^{+}$la partie de $T_{A}$ définie au moyen de ces racines comme il a été dit au $\mathrm{n}^{\circ} 10$ du Chapitre $\mathrm{I}$; d'après les résultats de ce $\mathrm{n}^{\circ}$, il y a une partie compacte $C_{1}$ de $P s_{A}$ telle que l'on ait $P s_{A}=C_{1} \cdot T_{A}^{+} \cdot P s_{k}$. Soit $T_{A}^{\prime}$ la partie de $T_{A}$ formée des éléments $d\left(\lambda_{t}\right)$ de $T_{A}$ pour lesquels on a

$$
\left|t_{1}\right|_{A} \geqslant \ldots \geqslant\left|t_{n}\right|_{A} \geqslant 1
$$

Pour $\varepsilon>0$, on vérifie aussitôt qu'on a $T_{A}^{+}=T_{A}^{\prime-1}$, de sorte qu'en posant $C=C_{1}^{-1}$, on aura $P s_{A}=P s_{k} \cdot T_{A}^{\prime} \cdot C$. Si $\varepsilon=0$, appliquons les résultats obtenus au sujet de $P s$ au $n^{\circ} 20$ du Chapitre II, où il faut alors prendre $\mathfrak{l}=k$ et $\eta=-1$; on voit que la matrice

$$
s_{1}=\left(\begin{array}{llll}
1_{n-1} & 0 & 0 & 0 \\
0 & 0 & 0 & 1 \\
0 & 0 & 1_{n-1} & 0 \\
0 & 1 & 0 & 0
\end{array}\right)
$$


appartient à $P s_{k}$; c'est d'ailleurs l'élément de $P s_{k}$ qui, avec nos notations habituelles, s'écrit $e^{\prime} \otimes d^{\prime}\left(\gamma^{\prime \prime}\right)$ si on écrit $X_{k}$ comme somme directe des modules $X_{k}^{\prime}, X_{k}^{\prime \prime}$ respectivement formés des éléments $\left(x_{1}, \ldots, x_{n-1}, 0\right)$ et $\left(0, \ldots, 0, x_{n}\right)$ et qu'on prenne pour $e^{\prime}$ l'élément neutre de $P s\left(X_{k}^{\prime} / \mathcal{A}_{k}\right)$ et pour $\gamma^{\prime \prime}$ l'isomorphisme évident de $\left(X_{k}^{\prime \prime}\right)^{*}$ sur $X_{k}^{\prime \prime}$ quand on identifie $X_{k}^{\prime \prime}$ et $\left(X_{k}^{\prime \prime}\right)^{*}$ avec $M_{m, 1}(\mathfrak{f})$ comme il a été dit au $n^{\circ} 20 \mathrm{du}$ Chapitre II. Cela posé, on vérifie aussitôt que $T_{A}^{+}$est la réunion de $T_{A}^{\prime-1}$ et de $s_{1}^{-1} T_{A}^{\prime-1} s_{1}$. Il s'ensuit qu'on aura de nouveau $P s_{A}=$ $P s_{k} \cdot T_{A}^{\prime} \cdot C$ à condition de prendre $C=C_{1}^{-1} \cup s_{1} C_{1}^{-1}$.

Par abus de langage, on conviendra dans ce qui suit d'identifier $\left(I_{k}\right)^{n}$ avec $T_{A}$ au moyen de l'isomorphisme $t \rightarrow d\left(\lambda_{t}\right)$, et aussi avec son image dans $M p_{A}$ au moyen de l'isomorphisme $t \rightarrow \mathbf{d}\left(\lambda_{t}\right)$, chaque fois que cela ne risquera pas d'entraîner de confusion. Avec cette convention, on peut donc écrire $M p_{A}=P s_{k} \cdot T_{A}^{\prime} \cdot \pi^{-1}(C)$, où $\pi$ est comme d'habitude la projection canonique de $M p_{A}$ sur $P s_{A} ; \pi^{-1}(C)$ est alors une partie compacte de $M p_{A}$. Cela entraîne le lemme suivant :

LEMмE 20. Soit $\hat{E}$ une mesure tempérée sur $X_{A}$, invariante par $P s_{k}$; soit $T_{A}^{\prime \prime}$ une partie de $T_{A}^{\prime}$ telle qu'on ait $T_{A}^{\prime} \subset T_{k} \cdot T_{A}^{\prime \prime} \cdot C^{\prime}, C^{\prime}$ étant une partie compacte de $T_{A}$. Alors, pour que la fonction $\mathbf{S} \rightarrow \hat{E}(\mathbf{S} \Phi)$ soit bornée sur $M p_{A}$, uniformément en $\Phi$ sur toute partie compacte de $\boldsymbol{S}\left(X_{A}\right)$, il faut et il suffit qu'elle le soit sur $T_{A}^{\prime \prime}$.

48. Soit $\mu^{\prime}$ une mesure (tempérée ou non) sur $X_{A}$. Pour qu'elle satisfasse à la condition (a) $\mathrm{du} \mathrm{n}^{\circ} 45$, Chapitre IV, ou autrement dit pour qu'elle soit invariante par $\mathrm{t}(q)$ chaque fois que $q \in Q\left(X_{k} / \mathcal{A}_{k}\right)$, il faut et il suffit évidemment que son support soit contenu dans l'ensemble des points $x$ de $X_{A}$ tels que $\chi(q(x))=1$ quel que soit $q \in Q\left(X_{k} / \mathcal{A}_{k}\right)$. Si on identifie $Q\left(X_{k} / \mathcal{A}_{k}\right)$ avec $I(X)_{k}^{*}$ comme il a été dit au $\mathrm{n}^{\circ} 15 \mathrm{du}$ Chapitre II, cette dernière condition revient à dire qu'on a $\chi\left(\left[i_{X}(x), i^{*}\right]\right)=1$ quel que soit $i^{*} \in I(X)_{k}^{*}$, ou encore $i_{X}(x) \in I(X)_{k}$. Autrement dit, la condition (a) est équivalente à la suivante :

(a') le support de $\mu^{\prime}$ est contenu dans la réunion des ensembles $i_{X}^{-1}(\{i\})$ pour $i \in I(X)_{k}$.

Cela peut aussi s'exprimer en disant que $\mu^{\prime}$ est somme de ses restrictions $\mu_{i}^{\prime}$ aux ensembles $i_{X}^{-1}(\{i\})$ pour $i \in I(X)_{k}$.

Supposons qu'en même temps $\mu^{\prime}$ satisfasse à condition (b) du $\mathrm{n}^{\circ} 45$, Chapitre IV, c'està-dire soit invariante par $x \rightarrow x \lambda$ quel que soit $\lambda \in \operatorname{Aut}\left(X_{k}\right)$; alors, si $\bar{\lambda}$ est l'automorphisme de $I(X)_{k}$ déterminé par $\lambda, \lambda$ détermine la permutation $\bar{\lambda}$ sur l'ensemble des mesures $\mu_{i}^{\prime}$, c'est-à-dire qu'on a, pour $i \in I(X)_{k}, i^{\prime}=i \bar{\lambda}$ :

$$
d \mu_{i}^{\prime}\left(x \lambda^{-1}\right)=d \mu_{i}^{\prime}(x) .
$$

D'autre part, identifiant comme plus haut $I(X)_{k}$ avec un sous-espace de $M_{n}(\mathfrak{f})$, convenons, pour tout $i \in I(X)_{k}$, de désigner par $i_{1}, \ldots, i_{n}$ les colonnes de la matrice $i$, et d'écrire 
$i=\left(i_{1}, \ldots, i_{n}\right)$. On aura donc $i_{\alpha} \in M_{n, 1}(\mathfrak{f})$ pour $1 \leqslant \alpha \leqslant n$; si $i=i_{X}(x)$, on a $i_{\alpha}={ }^{t} x^{\prime} \cdot h \cdot x_{\alpha}$. Pour $0 \leqslant \alpha \leqslant n$, on désignera par $I_{k}^{(\alpha)}$ l'ensemble des éléments $i=\left(i_{1}, \ldots, i_{n}\right)$ de $I(X)_{k}$ tels que $i_{1}=$ $\ldots=i_{\alpha}=0$ et $i_{\alpha+1} \neq 0 ; I(X)_{k}$ est donc réunion disjointe des $I_{k}^{(\alpha)}$ pour $0 \leqslant \alpha \leqslant n$.

Lемме 21. Soit $\hat{E}$ une mesure tempérée positive, invariante par $T_{k}$, dont le support soit contenu dans la réunion des $i_{X}^{-1}(\{i\})$ pour $i \in I_{k}^{(0)}$. Alors la fonction $\mathbf{S} \rightarrow \hat{E}(\mathbf{S} \Phi)$ est bornée sur $T_{A}^{\prime}$, uniformément en $\Phi$ sur toute partie compacte de $S\left(X_{A}\right)$.

Comme au $\mathrm{n}^{\circ} 9$ du Chapitre I, désignons par $\Theta(T)$ l'ensemble des éléments de $T_{A}$ de la forme $\left(a_{\tau_{1}}, \ldots, a_{\tau_{n}}\right)$, avec $\tau_{\alpha} \in \mathbf{R}_{+}^{*}$ pour $1 \leqslant \alpha \leqslant n$; posons $\Theta^{\prime}=\Theta(T) \cap T_{A}^{\prime} ; \Theta^{\prime}$ est l'ensemble des éléments de la forme ci-dessus pour lesquels $\tau_{1} \geqslant \ldots \geqslant \tau_{n} \geqslant 1$. II y a évidemment une partie compacte $C^{\prime}$ de $T_{A}^{\prime}$ telle que $T_{A}^{\prime}=T_{k} \cdot \Theta^{\prime} \cdot C^{\prime}$. Soit $C_{0}$ une partie compacte de $S\left(X_{A}\right)$; soit $C_{0}^{\prime}$ l'ensemble des $\mathrm{S} \Phi$ pour $\mathrm{S} \in C^{\prime}, \Phi \in C_{0}$. Appliquons le lemme 6 du Chapitre $\mathrm{I}, \mathrm{n}^{\circ} 13$, aux espaces $X_{k}=M_{m, n}(\mathfrak{k}), X_{k}^{(\alpha)}=M_{m, 1}(\mathfrak{k})$ pour $1 \leqslant \alpha \leqslant n, Y_{k}=M_{n, 1}(\mathfrak{f})$, et au morphisme $x \rightarrow p(x)={ }^{t} x^{\prime} \cdot h \cdot x_{1}$ de $X$ dans $Y$; on en conclut qu'il y a $\Phi_{0} \in S\left(X_{A}\right)$ tel que l'on ait $|\mathbf{S} \Phi| \leqslant \Phi_{0}$ sur le support de $\hat{E}$ quels que soient $S \in \Theta^{\prime}, \Phi \in C_{0}^{\prime}$. La conclusion du lemme s'ensuit aussitôt.

Le lemme 21 est particulièrement utile dans le cas $n=1$, puisqu'alors $I(X)_{k}=I_{k}^{(0)} \cup\{0\}$. On obtient par exemple ainsi le résultat suivant :

Proposition 7. Soient $\mathcal{A}_{k}$ de type (I) et $X_{k}$ de rang 1 , satisfaisant à (B), c'est-à-dire à $m>4 \varepsilon$. Soit $v$ une mesure positive à support compact sur $G_{A} / G_{k}$, telle que $\nu\left(G_{A} / G_{k}\right)=1$. Alors la formule

$$
E^{\prime}(\Phi)=\int_{G_{A} / G_{k}} \sum_{\xi \in X_{k}} \Phi(u \xi) \cdot d v(u)
$$

définit une mesure tempérée positive $E^{\prime}$ sur $X_{A}$, invariante par $P s_{k}$; et la fonction

$$
\mathbf{S} \rightarrow E^{\prime}(\mathbf{S} \Phi)-E(\mathbf{S} \Phi)
$$

est bornée sur $M p_{A}$, uniformément en $\Phi$ sur toute partie compacte de $S\left(X_{A}\right)$.

Soit $C$ une partie compacte de $G_{A}$ dont l'image dans $G_{A} / G_{k}$ contienne le support de $v$; soit $C_{0}$ une partie compacte de $S\left(X_{A}\right)$. D'après le lemme 5 de [14], $\mathrm{n}^{0} 41$, il y a $\Phi_{0} \in S\left(X_{A}\right)$ telle que $|\Phi(u x)| \leqslant \Phi_{0}(x)$ quels que soient $\Phi \in C_{0}, u \in C, x \in X_{A}$. L'intégrale qui définit $E^{\prime}(\Phi)$ est évidemment majorée par l'intégrale analogue étendue à $C$, avec $\Phi$ remplacée par $|\Phi|$, et l'est donc à plus forte raison par $v(C) \sum \Phi_{0}(\xi)$ pour $\Phi \in C_{0}$. D'après le lemme 2 du Chapitre $\mathrm{I}, \mathrm{n}^{\circ} 2$, elle définit donc une distribution tempérée $E^{\prime}$, qui est évidemment une mesure positive. L'invariance de $E^{\prime}$ par $P s_{k}$ est une conséquence immédiate du théorème 6 de [14], $\mathrm{n}^{\circ} 41$, joint à la proposition 9 de [14], $\mathrm{n}^{0} 51$. Ecrivons maintenant

$$
\hat{E}(\Phi)=\int_{G_{\mathbf{A}} / G_{\boldsymbol{k}}} \sum_{\xi \in X_{\mathbf{k}}-\{0\}} \Phi(u \xi) \cdot d \nu(u),
$$


ce qui donne $E^{\prime}=\hat{E}+\delta_{0}$ en vertu de l'hypothèse faite sur $v$. Appliquons le lemme 6 du Chapitre I, $\mathrm{n}^{\circ} 13$, en y prenant $n=1, X_{k}=Y_{k}, p$ étant l'application identique de $X$ sur $X$. Raisonnant sur $\hat{E}(\Phi)$ comme on a fait tout à l'heure sur $E^{\prime}(\Phi)$, on en conclut qu'il y a $\Phi_{0} \in S\left(X_{A}\right)$ telle que $\hat{E}(\mathrm{~S} \Phi)$ soit majorée par $\nu(C) \sum \Phi_{0}(\xi)$ chaque fois que $S \in \Theta^{\prime}$ et $\Phi \in C_{0}$. D'autre part, appliquons à $E$ les théorèmes 2 et 3 du Chapitre IV, $n^{\circ} 44$; avec les notations de ces théorèmes, on a d'abord $E=E_{X}+\delta_{0}$, puis $E_{X}=\mu_{0}+\hat{E}_{X}$, où $\hat{E}_{X}$ a son support dans la réunion des ensembles $i_{X}^{-1}(\{i\})$ pour $i \in I(X)_{k}, i \neq 0$. On peut donc appliquer à $\hat{E}_{X}$ le lemme 21 , qui montre que $\hat{E}_{X}(\mathrm{~S} \Phi)$ reste borné pour $\mathrm{S} \in \Theta^{\prime}, \Phi \in C_{0}$. Enfin, comme $\mu_{0}$ est la mesure déterminée par la jauge $\theta_{0}$ sur $U(0)_{A}$, on a, d'après la formule (36) du $\mathbf{n}^{\circ} 46$ :

$$
d \mu_{0}\left(x \lambda_{t}^{-1}\right)=|t|_{A}^{(-m+2 \varepsilon) \delta} d \mu_{0}(x)
$$

pour $t \in I_{k}$, et par suite, d'après la formule (37) du $\mathrm{n}^{\circ} 46$ :

$$
\int \mathrm{d}\left(\lambda_{t}\right) \Phi \cdot d \mu_{0}=|t|_{A}^{-(m-4 \varepsilon) \delta / 2} \int \Phi \cdot d \mu_{0}
$$

Puisque par hypothèse on a $m>4 \varepsilon$, cette expression reste bornée pour $|t|_{A} \geqslant 1$, ou autrement dit sur $T_{A}^{\prime}$, uniformément en $\Phi$ sur toute partie compacte de $S\left(X_{A}\right)$. Comme on a $E^{\prime}-E=\hat{E}-\mu_{0}-\hat{E}_{X}$, il s'ensuit que $E^{\prime}-E$ satisfait aux conditions du lemme $20 \mathrm{du} \mathrm{n}^{\circ} 47$, ce qui achève la démonstration.

La proposition 7 s'applique par exemple au cas où on a pris $\nu=\delta_{1}$, c'est-à-dire $E^{\prime}(\Phi)=$ $\sum \Phi(\xi)$. Le résultat qu'on obtient ainsi comprend comme cas particuliers les théorèmes classiques d'après lesquels la partie principale d'une forme modulaire définie par une série thêta est donnée par une série d'Eisenstein (ou, ce qui revient au même, les résultats sur la " série singulière » qui donne la partie principale du nombre de représentations d'un entier par une forme quadratique).

49. Comme le montre l'exemple de la mesure $\delta_{0}$, on n'a pas le droit de remplacer $I_{k}^{(0)}$ par $I(X)_{k}$, ni par $I_{k}^{(\alpha)}$ pour $\alpha>0$, dans le lemme 21, à moins d'introduire des hypothèses supplémentaires sur $\hat{E}$; c'est ce qui va être fait dans le lemme 23.

Pour nous exprimer plus commodément, nous ferons la convention suivante. Soit $i \in I(X)_{k}$; soit $j$ l'injection canonique de $U(i)$ dans $X$, qui est un morphisme de variétés algébriques; $j$ détermine alors une application injective $j_{A}$ de $U(i)_{A}$ dans $X_{A}$, et plus précisément dans $i_{X}^{-1}(\{i\})$. Nous dirons alors qu'une mesure dans $X_{A}$ est portée par $U(i)_{A}$ si c'est l'image par $j_{A}$ d'une mesure sur $U(i)_{A}$. Par exemple, il en est ainsi, par définition, de la mesure $\mu_{i}$, image de $\left|\theta_{i}\right|_{A}$ par $j_{A}$, qui figure dans le théorème 2 du Chapitre IV, $\mathrm{n}^{\circ} 44$. Dans le cas où $i$ est un élément non-dégénéré de $I(X)_{k}$, il résulte des remarques du Cha- 
pitre II, $\mathbf{n}^{\circ} 25$, que $j_{A}$ est un isomorphisme de $U(i)_{A}$ sur $i_{X}^{-1}(\{i\})$; dans ce cas, toute mesure de support contenu dans $i_{X}^{-1}(\{i\})$ est portée par $U(i)_{A}$.

D'autre part, dans ce qui suit, on se donnera une fois pour toutes une place $v$ de $k$, et on écrìra $X_{A}=X_{v} \times X^{\prime}$, où $X^{\prime}$ est défini à partir des $X_{w}$ et des $X_{w}^{\circ}$, pour $w \neq v$, comme $X_{A}$ l'est à partir des $X_{v}$ et des $X_{v}^{\circ}$; pour $x \in X_{A}$, on écrira $x=\left(x_{v}, x^{\prime}\right)$, où $x_{v}$ et $x^{\prime}$ sont les projections de $x$ sur $X_{v}$ et sur $X^{\prime}$. On écrira de même $U(i)_{A}=U(i)_{v} \times U(i)^{\prime}$.

L Емм 22. Soit $i \in I(X)_{k}$, et soit $G_{v}^{\prime}$ un sous-groupe de $G_{v}$ qui opère transitivement sur $U(i)_{v}$. Soit $\mu$ une mesure tempérée positive portée par $U(i)_{A}$ et invariante par $G_{v}^{\prime}$. Alors, à toute fonction $\Phi^{\prime} \in S\left(X^{\prime}\right)$, on peut faire correspondre $c\left(\Phi^{\prime}\right)$ de manière à avoir, quel que soit $\Phi_{v} \in \boldsymbol{S}\left(\boldsymbol{X}_{v}\right)$ :

$$
\int \Phi_{v}\left(x_{v}\right) \Phi^{\prime}\left(x^{\prime}\right) d \mu\left(\left(x_{v}, x^{\prime}\right)\right)=c\left(\Phi^{\prime}\right) \int_{U(i) v} \Phi_{v} \cdot\left|\theta_{i}\right|_{v}
$$

Par hypothèse, $\mu$ est image d'une mesure $\nu$ sur $U(i)_{A}$, c'est-à-dire que le premier membre de (38) est l'intégrale de $\Phi_{v}\left(x_{v}\right) \Phi^{\prime}\left(x^{\prime}\right)$ sur $U(i)_{v} \times U(i)^{\prime}$ par rapport à $\nu$. Supposons d'abord $\Phi^{\prime} \geqslant 0$. Par hypothèse, l'intégrale en question est finie chaque fois que $\Phi_{v}$ est $\geqslant 0$ et appartient à $S\left(X_{v}\right)$, donc aussi chaque fois que $\Phi_{v}$ est continue à support compact sur $X_{v}$, et à plus forte raison chaque fois que $\Phi_{v}$ est continue à support compact sur $U(i)_{v^{*}}$. Elle peut done s'écrire $\int \Phi_{v} d v_{v}$, où $v_{v}$ est une mesure positive sur $U(i)_{v}$. D'après l'hypothèse faite sur $\mu, \nu_{v}$ est invariante par $G_{v}^{\prime}$. On peut d'ailleurs supposer $G_{v}^{\prime}$ fermé dans $G_{v}$ (sinon, on le remplacerait par son adhérence), et par suite identifier $U(i)_{v}$ avec l'espace homogène déterminé par $G_{v}^{\prime}$ et le groupe de stabilité d'un de ses points dans $G_{v}^{\prime}$. Mais la définition de la jauge $\theta_{i}$ dans le théorème 2 du Chapitre IV, $n^{\circ} 44$, montre qu'elle est invariante par $G$, à un facteur \pm 1 près; par suite, la mesure $\left|\theta_{i}\right|_{v}$ est invariante par $G_{v}$, et à plus forte raison par $G_{v}^{\prime}$. Les théorèmes connus sur l'unicité de la mesure invariante dans les espaces homogènes (cf. p. ex. [4], $§ 2, \mathrm{n}^{\circ} 6$ ) montrent alors que $\nu_{v}$ ne peut différer de $\left|\theta_{i}\right|_{v}$ que par un facteur scalaire $c\left(\Phi^{\prime}\right)$. Le cas général où on ne suppose pas $\Phi^{\prime} \geqslant 0$ se ramène immédiatement au précédent.

Lемм 23. Soit $\hat{E}$ une mesure tempérée positive, invariante par $T_{k}$ et somme de mesures $\hat{\mu}_{i}$ respectivement portées par les $U(i)_{A}$ pour $i \in I(X)_{k}$. Supposons qu'il existe une place $v$ de $k$, et un sous-groupe $G_{v}^{\prime}$ de $G_{v}$ opérant transitivement sur $U(i)_{v} q u e l$ que soit $i \in I(X)_{k}$, tels que $\hat{E}$ soit invariante par $G_{v}^{\prime}$. Alors la fonction $\mathbf{S} \rightarrow \hat{E}(\mathbf{S} \Phi)$ est bornée sur $T_{A}^{\prime}$, uniformément en $\Phi$ sur toute partie compacte de $\mathfrak{S}\left(X_{A}\right)$.

Soit $\hat{E}_{\alpha}$, pour $0 \leqslant \alpha \leqslant n$, la somme des $\hat{\mu}_{i}$ pour $i \in I_{k}^{(\alpha)}$; on aura $\hat{E}=\hat{E}_{0}+\ldots+\hat{E}_{n}$. Si $t \in T_{k}, \bar{\lambda}_{t}$ détermine une permutation sur chacun des ensembles $I_{k}^{(\alpha)}$, de sorte que chacune 
des mesures $\hat{E}_{\alpha}$ est invariante par $T_{k}$. D'autre part, $G_{A}$ laisse invariant chacun des ensembles $i_{X}^{-1}(\{i\})$; avec les hypothèses de l'énoncé, il s'ensuit que $G_{v}^{\prime}$ laisse invariante chacune des mesures $\hat{\mu}_{i}$, donc aussi chacune des $\hat{E}_{\alpha}$; celles-ci satisfont donc aux mêmes hypothèses que $\hat{E}$, et on est ramené à faire la démonstration pour les $\hat{E}_{\alpha}$.

Soit donc $\alpha$ tel que $0 \leqslant \alpha \leqslant n$. Il y a une constante $q$, égale à $\mathbf{l}$ si $v$ est une place à l'infini et à $N\left(\mathfrak{p}_{v}\right)$ dans le cas contraire, telle qu'il existe, pour tout $\tau \in \mathbf{R}_{+}^{*}$, un élément $y$ de $k_{v}$ satisfaisant à $\tau \leqslant|y|_{v} \leqslant q \tau$; et il existe une partie compacte $C$ de $I_{k}$ telle que tout élément $t$ de $I_{k}$ satisfaisant à $1 \geqslant|t|_{A} \geqslant q^{-1}$ puisse s'écrire sous la forme $\varrho c$ avec $\varrho \in k, c \in C$; on notera $C^{n}$ la partie compacte de $T_{A}$ formée des éléments $\left(c_{1}, \ldots, c_{n}\right)$ avec $c_{\beta} \in C$ pour $1 \leqslant \beta \leqslant n$. Donnons-nous aussi une partie compacte $C_{0}$ de $S\left(X_{A}\right)$, et appliquons le lemme 6 du Chapitre $\mathrm{I}, \mathrm{n}^{0} 13$, à l'espace $X_{k}=M_{m, n}(\mathfrak{f})$ considéré comme produit des espaces

$$
X_{k}^{(1)}=M_{m, \alpha+1}(\mathfrak{f}), \quad X_{k}^{(2)}=\ldots=X_{k}^{(n-\alpha)}=M_{m, 1}(\mathfrak{f})
$$

de telle manière que les projections de $x=\left(x_{1}, \ldots, x_{n}\right)$ sur ces espaces soient respetivement $\left(x_{1}, \ldots, x_{\alpha+1}\right), x_{\alpha+2}, \ldots, x_{n}$; on prendra $Y_{k}=M_{n, \alpha+1}(\mathfrak{f})$, et $p$ sera le morphisme de $X$ dans $Y$ donné par

$$
p(x)={ }^{t} x^{\prime} \cdot h \cdot\left(x_{1}, \ldots, x_{\alpha+1}\right) .
$$

On en conclut qu'il existe $\Phi_{0} \in S\left(X_{A}\right)$ tel que l'on ait

$$
\left|\mathbf{d}\left(\lambda_{\theta}\right) \mathbf{d}\left(\lambda_{c}\right) \Phi(x)\right| \leqslant \Phi_{0}(x)
$$

chaque fois que $x \in X_{A}, i_{X}(x) \in I_{k}^{(\alpha)}, c \in C^{n}, \Phi \in C_{0}$, et que $\theta$ appartient à l'ensemble $\Theta_{\alpha}^{\prime}$ des éléments $\left(a_{\tau_{1}}, \ldots, a_{\tau_{n}}\right)$ de $\Theta(T)$ qui satisfont à la condition

$$
\tau_{1}=\ldots=\tau_{\alpha+1} \geqslant \ldots \geqslant \tau_{n} \geqslant 1 .
$$

De plus, on peut supposer que $\Phi_{0}$ a été pris de la forme $\Phi_{v}\left(x_{v}\right) \Phi^{\prime}\left(x^{\prime}\right)$, avec

$$
\Phi_{v} \in S\left(X_{v}\right), \Phi^{\prime} \in S\left(X^{\prime}\right) .
$$

Soit maintenant $t=\left(t_{1}, \ldots, t_{n}\right)$ un élément de $T_{A}^{\prime}$. Pour $1 \leqslant \beta \leqslant \alpha$, soit $y_{\beta} \in k_{v}$ tel que $\left|y_{\beta}\right|_{v}$ soit compris entre $\left|t_{\beta} t_{\alpha+1}^{-1}\right|_{A}$ et $q\left|t_{\beta} t_{\alpha+1}^{-1}\right|_{A}$; soit $y_{\beta}=1$ pour $\beta \geqslant \alpha+1$; on aura $\left|y_{\beta}\right|_{v} \geqslant 1$ pour $1 \leqslant \beta \leqslant n$. D'autre part, pour $\beta \geqslant \alpha+1$, soit $\tau_{\beta} \in \mathbf{R}_{+}^{*}$ tel que $\left|a_{\tau_{\beta}}\right|_{A}=\left|t_{\beta}\right|_{A}$, et soit $\tau_{\beta}=\tau_{\alpha+1}$ pour $1 \leqslant \beta \leqslant \alpha$. Pour tout $\beta$, on aura

$$
1 \geqslant\left|t_{\beta} y_{\beta}^{-1} a_{\tau_{\beta}}^{-1}\right|_{A} \geqslant q^{-1},
$$

de sorte qu'on pourra écrire $t_{\beta}=\varrho_{\beta} y_{\beta} a_{\tau_{\beta}} c_{\beta}$, avec $\varrho_{\beta} \in k, c_{\beta} \in C$, pour $1 \leqslant \beta \leqslant n$. En posant

$$
y=\left(y_{1}, \ldots, y_{n}\right), \quad \theta=\left(a_{\tau_{1}}, \ldots, a_{\tau_{n}}\right)
$$


on aura donc $t=\varrho y \theta c$ avec $\varrho \in T_{k}, y \in T_{v}, \theta \in \Theta_{\alpha}^{\prime}$ et $c \in C^{n}$. Comme $\hat{E}_{\alpha}$ est invariante par $T_{k}$, il s'ensuit qu'on a

$$
\left|\hat{E}_{\alpha}\left(\mathbf{d}\left(\lambda_{t}\right) \Phi\right)\right| \leqslant \hat{E}_{\alpha}\left(\mathbf{d}\left(\lambda_{y}\right) \Phi_{0}\right)
$$

chaque fois que $\Phi \in C_{0}, \Phi_{0}$ étant choisie comme ci-dessus.

Pour évaluer le second membre de cette inégalité, on appliquera le lemme 22 à chacune des mesures $\hat{\mu}_{i}$ pour $i \in I_{k}^{(\alpha)}$; en notant $c_{i}\left(\Phi^{\prime}\right)$ la constante qui apparait au second membre de (38) lorsqu'on substitue $\hat{\mu}_{i}$ à $\mu$ au premier membre, on obtient :

$$
\hat{E}_{\alpha}\left(\mathbf{d}\left(\lambda_{y}\right) \Phi_{0}\right)=\sum_{i \in I_{k}^{(\alpha)}} c_{i}\left(\Phi^{\prime}\right) \int_{U(i)_{v}} \mathrm{~d}\left(\lambda_{y}\right) \Phi_{v} \cdot\left|\theta_{i}\right|_{v} .
$$

Comme on a $y_{\beta}=1$ pour $\beta \geqslant \alpha+1$, il résulte de la définition de $I_{k}^{(\alpha)}$ que l'automorphisme $\bar{\lambda}_{y}$ de $I(X)_{v}$ déterminé par $\lambda_{y}$ laisse invariants tous les éléments de $I_{k}^{(\alpha)}$. En appliquant aux termes du second membre de la relation ci-dessus les formules (36) et (37) du $\mathrm{n}^{\circ} 46$, on obtient alors

$$
\hat{E}_{\alpha}\left(\mathbf{d}\left(\lambda_{y}\right) \Phi_{0}\right)=\left|y_{1} \ldots y_{\alpha}\right|_{v}^{(-m+2 n+4 \varepsilon-2) \delta / 2} \hat{E}_{\alpha}\left(\Phi_{0}\right)
$$

Comme on a supposé $(\mathbf{B})$ satisfaite, l'exposant du second membre est $<0$. Comme on a $\left|y_{\beta}\right|_{v} \geqslant 1$ quel que soit $\beta$, on obtient en définitive

$$
\left|\hat{E}_{\alpha}\left(\mathrm{d}\left(\lambda_{t}\right) \Phi\right)\right| \leqslant \hat{E}_{\alpha}\left(\Phi_{0}\right)
$$

cette inégalité étant valable chaque fois que $t \in T_{A}^{\prime}$ et $\Phi \in C_{0}$. Cela achève la démonstration.

50. Nous sommes maintenant en mesure de démontrer le résultat principal que nous avions en vue dans ce chapitre.

THÉoR ÈmE 4. Soient $\mathcal{A}_{k}$ une algèbre de type (I) et $X_{k}$ un $\mathcal{A}_{k}$-module à gauche, satisfaisant à $(\mathbf{B})$, c'est-à-dire à $m>2 n+4 \varepsilon-2$. Soient $v$ une place de $k$ telle que $U(0)_{v}$ ne soit pas vide, et $G_{v}^{\prime}$ un sous-groupe de $G_{v}$ opérant transitivement sur $U(i)_{v}$ quel que soit $i \in I(X)_{k}$. Soit $E^{\prime}$ une mesure tempérée positive sur $X_{A}$, invariante par $P s_{k}=P s\left(X_{k} / \mathcal{A}_{k}\right)$ et par $G_{v}^{\prime}$, et telle que $E^{\prime}-E$ soit somme de mesures portées par les $U(i)_{A}$ pour $i \in I(X)_{k^{*}}$. Alors on a $E^{\prime}=E$.

Avec les notations des théorèmes 2 et 3 du Chapitre IV, $\mathrm{n}^{\circ} 44$, on a $E=\sum E_{z}$, la som. mation étant étendue à tous les sous-modules $Z_{k}$ de $X_{k} ; E_{X}$ est somme des mesures $\left|\theta_{i}\right|_{A}$ respectivement portées par les $U(i)_{A}$, tandis que $E_{Z}$ a son support contenu dans $Z_{A}$ quel que soit $Z_{k} \neq X_{k}$. Sur le domaine universel, soit $U$ l'ensemble des points de $X$ de rang maximal; il est $k$-ouvert; c'est une orbite pour le groupe des éléments inversibles de $\mathcal{A}$; quel que soit $i \in I(X)_{k}, U(i)$ est une sous-variété de $U$ et est donc $k$-fermée dans $U$. Soit 
$F=X-U$; c'est une partie $k$-fermée de $X$, invariante par le groupe des éléments inversibles de $\mathcal{A}$ et à plus forte raison par $G$, et qui contient $Z$ chaque fois que $Z_{k} \neq X_{k}$. Par suite, $F_{A}$ est une partie fermée de $X_{A}$, invariante par $G_{A}$ et évidemment aussi par $\operatorname{Aut}\left(X_{k}\right)$, qui contient $Z_{A}$ pour $Z_{k} \neq X_{k}$, et qui est sans point commun avec $U(i)_{A}$ quel que soit $i \in I(X)_{k}$. Il s'ensuit que $E_{X}$ est la restriction de $E$ à l'ensemble ouvert $X_{A}-F_{A}$, et que la somme $\sum E_{Z}$, étendue à tous les $Z_{k} \neq X_{k}$, est la restriction de $E$ à $F_{A}$. L'hypothèse faite sur $E^{\prime}$ implique alors que la restriction $\hat{E}$ de $E^{\prime}$ à $X_{A}-F_{A}$ est somme de mesures $\hat{\mu}_{i}$ respectivement portées par les $U(i)_{A}$ pour $i \in I(X)_{k}$, et que la restriction de $E^{\prime}$ à $F_{A}$ est la même que celle de $E$, de sorte qu'on a $E^{\prime}-\widehat{E}=E-E_{X}$; de plus, comme $E^{\prime}$ et $F_{A}$ sont invariants par $G_{v}^{\prime}$ et par Aut $\left(X_{k}\right)$, il en est de même de $\hat{E}$, qui satisfait donc aux hypothèses du lemme $23 \mathrm{du} \mathrm{n}^{\circ} 49$.

D'après ce lemme, la fonction $\mathbf{S} \rightarrow \hat{E}(\mathbf{S} \Phi)$ est donc bornée sur $T_{A}^{\prime}$, uniformément en $\Phi$ sur toute partie compacte de $S\left(X_{A}\right)$. Cette conclusion s'applique en particulier à $E_{X}$, qui est déduit de $E$ comme $\hat{E}$ l'est de $E^{\prime}$; elle s'applique donc aussi à la mesure tempérée $E^{\prime \prime}$ donnée par

$$
E^{\prime \prime}=E^{\prime}-E=\hat{E}-E_{X}
$$

Mais celle-ci est invariante par $P s_{k}$, puisqu'il en est ainsi de $E$, et aussi, par hypothèse, de $E^{\prime}$; on peut donc lui appliquer le lemme 20 du $\mathrm{n}^{\circ} 47$, qui montre que la fonction $\mathrm{S} \rightarrow E^{\prime \prime}(\mathrm{S} \Phi)$ est bornée sur $M p_{A}$, quelle que soit $\Phi \in S\left(X_{A}\right)$. Pour tout $\Phi \in S\left(X_{A}\right)$, on désignera par $M(\Phi)$ la borne supérieure de $\left|E^{\prime \prime}(\mathbf{S} \Phi)\right|$ pour $\mathbf{S} \in M p_{A}$; on aura $M(\mathrm{~S} \Phi)=M(\Phi)$ quel que soit $\mathbf{S} \in M p_{A}$.

La mesure $E^{\prime \prime}$ est somme des mesures $\mu_{i}^{\prime \prime}=\hat{\mu}_{i}-\mu_{i}$, où $\mu_{i}$ désigne de nouveau la mesure $\left|\theta_{i}\right|_{A}$ portée par $U(i)_{A}$. On a donc, pour $\Phi \in \mathcal{S}\left(X_{A}\right)$ :

$$
E^{\prime \prime}(\Phi)=\sum_{i \in I(X)_{k}} \int \Phi d \mu_{i}^{\prime \prime}
$$

dans cette formule, la série du second membre est absolument convergente, uniformément en $\Phi$ sur toute partie compacte de $S\left(X_{A}\right)$, puisqu'il en est évidemment ainsi des séries analogues formées au moyen des mesures positives $\hat{\mu}_{i}$ et $\mu_{i}$. Soit $i^{*} \in I(X)_{A}^{*}$; soit $q$ l'élément de $Q(X / \mathcal{A})_{A}$ qui correspond à $i^{*}$ en vertu des isomorphismes du Chapitre II, $\mathrm{n}^{\circ} 15$, c'est-àdire qui est défini par $q(x)=\left[i_{X}(x), i^{*}\right]$ pour $x \in X_{A}$. On a alors, pour $\Phi \in S\left(X_{A}\right)$ :

et par suite

$$
\mathbf{t}(q) \Phi(x)=\Phi(x) \chi(q(x))=\Phi(x) \chi\left(\left[i_{X}(x), i^{*}\right]\right)
$$

$$
E^{\prime \prime}(\mathbf{t}(q) \Phi)=\sum_{i \in I(X) k} \chi\left(\left[i, i^{*}\right]\right) \int \Phi d \mu_{i}^{\prime \prime} .
$$

On peut considérer cette formule comme donnant le développement du premier membre 
en série de Fourier sur le groupe compact $I(X)_{A}^{*} / I(X)_{k}^{*}$. Comme le premier membre, en valeur absolue, est $\leqslant M(\Phi)$, on aura, en vertu des formules de Fourier

$$
\left|\int \Phi d \mu_{i}^{\prime \prime}\right| \leqslant M(\Phi)
$$

et par suite, en remplaçant $\Phi$ par $\mathbf{S} \Phi$ :

$$
\left|\int \mathbf{S} \Phi \cdot d \mu_{i}^{\prime \prime}\right| \leqslant M(\Phi)
$$

cette inégalité étant valable quels que soient $\mathbf{S} \in M p_{A}, i \in I(X)_{k}$ et $\Phi \in S\left(X_{A}\right)$.

Prenons $\Phi$ de la forme $\Phi_{v}\left(x_{v}\right) \Phi^{\prime}\left(x^{\prime}\right)$, avec $\Phi_{v} \in S\left(X_{v}\right), \Phi^{\prime} \in S\left(X^{\prime}\right)$. En vertu des hypothèses faites sur $E^{\prime}$, les $\hat{\mu}_{i}$ sont invariantes par $G_{v}^{\prime}$, et il en est bien entendu de même des $\mu_{i}$; on peut donc leur appliquer le lemme $22 \mathrm{du} \mathrm{n}^{\circ} 49$. Par suite, on peut écrire

$$
\int \Phi d \mu_{i}^{\prime \prime}=c_{i}\left(\Phi^{\prime}\right) \int_{v(i)_{v}} \Phi_{v} \cdot\left|\theta_{i}\right|_{v}
$$

Dans cette formule, remplaçons $\Phi$ par $\mathbf{d}\left(\lambda_{t}\right) \Phi$, avec $t \in T_{v}$; cela revient à ne pas changer $\Phi^{\prime}$ et à remplacer $\Phi_{v} \operatorname{par} \mathbf{d}\left(\lambda_{t}\right) \Phi_{v}$, cette dernière fonction étant donnée par la formule analogue à $(37)$, relative à $X_{v}$. En posant $i^{\prime}=i \bar{\lambda}_{t}$, cela donne, d'après (36) :

$$
\int \mathbf{d}\left(\lambda_{t}\right) \Phi \cdot d \mu_{i}^{\prime \prime}=c_{t}\left(\Phi^{\prime}\right)\left|t_{1} \ldots t_{n}\right|_{v}^{(-m+2 n+4 \varepsilon-2) \delta / 2} \int_{U\left(i^{\prime}\right) v} \Phi_{v} \cdot\left|\theta_{i^{\prime}}\right|_{v} .
$$

Désignons par $F\left(i^{\prime}\right)$ l'intégrale qui figure au second membre; la proposition 6 du Chapitre III, $\mathrm{n}^{\circ} 37$, montre que c'est une fonction continue de $i^{\prime}$ dans $I(X)_{v}$, de sorte que $F\left(i^{\prime}\right)$ tend vers $F(0)$ quand on fait tendre tous les $\left|t_{\alpha}\right|_{v}$ vers 0 . Comme l'exposant de $\left|t_{1} \ldots t_{n}\right|_{v}$, au second membre de $(40)$, est $<0$ en vertu de la condition $(B)$, et que, d'après (39), ce second membre doit rester borné quel que soit $t \in T_{v}$, on en conclut qu'on doit avoir $c_{i}\left(\Phi^{\prime}\right) F(0)=0$. Mais $F(0)$ est donné par

$$
F(0)=\int_{U(0)_{v}} \Phi_{v} \cdot\left|\theta_{0}\right|_{v}
$$

et, par hypothèse, $U(0)_{v}$ n'est pas vide; on peut donc choisir $\Phi_{v}$ de manière que $F(0)$ ne soit pas nul. On a donc $c_{i}\left(\Phi^{\prime}\right)=0$, et par suite $\int \Phi d \mu^{\prime \prime}=0$ chaque fois que $\Phi$ est de la forme $\Phi_{v}\left(x_{v}\right) \Phi^{\prime}\left(x^{\prime}\right)$. Cela entraîne évidemment $\mu_{i}^{\prime \prime}=0$. Comme il en est ainsi quèl que soit $i \in I(X)_{k}$, on a donc $E^{\prime \prime}=0$, c'est-à-dire $E^{\prime}=E$.

On observera que le théorème 4 fournit une caractérisation de la mesure $E_{X}$, par récurrence sur le rang $n$ de $X_{k}$, à partir de $E_{0}=\delta_{0}$. 


\section{La formule de Siegel}

51. La "formule de Siegel », au sens où nous l'entendons ici, donne la relation entre la "série d'Eisenstein-Siegel " $E(\Phi)$ qu'on a introduite et étudiée au Chapitre IV, et certaines intégrales du type considéré au Chapitre $\mathrm{I}, \mathrm{n}^{\mathrm{os}} 7,8$ et 12 .

L'algèbre à involution $\mathcal{A}_{k}$ et le module $X_{k}$ étant donnés comme précédemment, on continuera à désigner par $\mathcal{A}$ l'extension de $\mathcal{A}_{k}$ au domaine universel et par $G$ le groupe réductif des éléments $u$ de $\mathcal{A}$ tels que $u \cdot u^{\imath}=1$. On désignera par $\varrho$ la représentation de $G$ (dans le groupe des automorphismes de l'espace vectoriel sous-jacent à $X$ ) qui est donnée par $\varrho(u) x=u x$. On considérera de nouveau le sous-groupe $G_{1}$ de $G$ qui a été défini au $\mathrm{n}^{\circ} 28$ du Chapitre II; on notera $\varphi_{1}$ l'injection canonique de $G_{1}$ dans $G$, et $\varrho_{1}$ la représentation de $G_{1}$ définie par $\varrho_{1}=\varrho \circ \varphi_{1}$. Lorsque $\mathcal{A}_{k}$ est de type $\left(\mathrm{I}_{4}\right)$ avec $m \geqslant 3$, ou de type $\left(\mathrm{I}_{3}\right)$ avec $m \geqslant 2$, on désignera par $\tilde{G}$ le revêtement simplement connexe de $G_{1}$ (le groupe "spin "), par $\tilde{\varphi}_{1}$ l'homomorphisme canonique de $\tilde{G}$ sur $G_{1}$ (dont le noyau est un groupe à deux éléments), et on posera $\tilde{\varphi}=\varphi_{1} \circ \tilde{\varphi}_{1}, \tilde{\varrho}=\varrho \circ \tilde{\varphi}$.

Avec ces notations, écrivons, pour $\Phi \in \boldsymbol{S}\left(X_{A}\right)$ :

$$
I(\Phi)=\int_{G_{A} / G_{k}} \sum_{\xi \in X_{k}} \Phi(\varrho(u) \xi) \cdot d \mu(u)
$$

où $\mu$ est une mesure de Haar sur $G_{A}$. Pour $\Phi \geqslant 0, \Phi(0)=1$, cette intégrale est $\geqslant \mu\left(G_{A} / G_{k}\right)$; pour qu'elle converge quel que soit $\Phi \in S\left(X_{A}\right)$, il faut done que $G_{A} / G_{k}$ soit de mesure finie. Dans ces conditions, on conviendra de normer $\mu$ par la condition $\mu\left(G_{A} / G_{k}\right)=1$.

On désignera par $I_{1}(\Phi)$ et $\tilde{I}(\Phi)$ les intégrales obtenues en substituant, d'une part $G_{1}$ et $\varrho_{1}$, d'autre part (lorsque $\widetilde{G}$ est défini) $\widetilde{G}$ et $\tilde{\varrho}$ à $G$ et $\varrho$.

On va d'abord déterminer les cas où ces intégrales satisfont aux conditions suffisantes de convergence contenues dans le lemme 5 du Chapitre $I, \mathrm{n}^{\circ} 12$; on se bornera pour cela aux cas où $\mathcal{A}_{k}$ est de type (I) ou de type (II). Dans le cas (I), avec nos notations habituelles, l'involution $\iota$ sur $\mathcal{A}_{k}$ est définie au moyen d'une forme $\eta$-hermitienne $h$ sur $A \times A, A$ étant un espace vectoriel à droite de dimension $m$ sur $\mathfrak{f}$; on désignera par $r$ l'indice de $h$, c'està-dire la dimension sur $\mathfrak{f}$ d'un sous-espace totalement isotrope maximal pour $h$ dans $A$.

Proposition 8. Si $\mathcal{A}_{k}$ est de type (I), l'intégrale $I(\Phi)$ est absolument convergente, quelle que soit $\Phi \in S\left(X_{A}\right)$, chaque fois que $r=0$ ou que $m-r>n+2 \varepsilon-1$; il en est de même de $I_{1}(\Phi)$, et de $\tilde{I}(\Phi)$ quand $\tilde{G}$ est défini. Si $A_{k}$ est de type $(\mathrm{II}), I_{1}(\Phi)$ est absolument convergente quelle que soit $\Phi \in S\left(X_{A}\right)$, chaque fois que $m=1$ ou que $m>p+q$.

L'assertion est triviale quand $G$ (resp. $G_{1}, \tilde{G}$ ) est " anisotrope ", c'est-à-dire ne contient aucun tore trivial, puisqu'alors $G_{A} / G_{k}$, ou l'espace analogue pour $G_{1}$ ou $\tilde{G}$, est compact. Il 
en est ainsi, comme on sait, pour $r=0$ dans le cas (I), et pour $m=1$, en ce qui concerne le groupe $G_{1}$, dans le cas (II). Ces cas étant laissés de côté, plaçons-nous d'abord dans le cas (I); $h$ étant d'indice $r>0$, on sait qu'on peut choisir une base dans $A$ pour laquelle $h$ soit donnée par une matrice de la forme

$$
h=\left(\begin{array}{ccc}
0 & 0 & 1_{r} \\
0 & h_{0} & 0 \\
\eta \cdot 1_{r} & 0 & 0
\end{array}\right),
$$

où $h_{0}$ est la matrice (d'ordre $m-2 r$ ) d'une forme $\eta$-hermitienne d'indice 0 (ou " anisotrope "); cela implique qu'on a $m \geqslant 2 r$. Pour $t=\left(t_{1}, \ldots, t_{r}\right) \in\left(G_{m}\right)^{r}$, désignons par $f(t)$ la matrice diagonale d'ordre $m$ dont les éléments diagonaux sont

$$
\left(t_{1}, \ldots, t_{r}, 1, \ldots, 1, t_{1}^{-1}, \ldots, t_{r}^{-1}\right)
$$

Il est bien connu que $f$ est alors un isomorphisme de $\left(G_{m}\right)^{r}$ sur un tore trivial maximal $T$ de $G ; T$ est aussi un tore trivial maximal de $G_{1}$, et un tore trivial maximal $\widetilde{T}$ de $\tilde{G}$, dans les cas où $\tilde{G}$ est défini, est donné par $\tilde{T}=\tilde{\varphi}^{-1}(T)$. Identifions $T$ avec $\left(G_{m}\right)^{r}$ au moyen de $f$, et désignons par $\delta$, comme toujours, la dimension de $\mathfrak{f}$ sur $k$. Alors il est aisé de voir qu'on peut ordonner les racines de $G$ de manière que les racines strictement positives (c'est-à-dire $\neq 1$ et $>1$ ) soient les suivantes : $t_{i} t_{j}^{-1}$ et $t_{i} t_{j}$ pour $1 \leqslant i<j \leqslant r$, chacune avec la multiplicité $\delta ; t_{i}$ et $t_{i}^{2}$ pour $1 \leqslant i \leqslant r$, avec les multiplicités respectives $\delta(m-2 r)$ et $\delta(1-\varepsilon)$. Les poids de $\varrho$ sont les $t_{i}$ et les $t_{i}^{-1}$ pour $l \leqslant i \leqslant r$, chacun avec la multiplicité $\delta n$. Les racines de $G_{1}$ et les poids de $\varrho_{1}$ sont les mêmes que ceux de $G$ et de $\varrho$; ceux de $\tilde{G}$ et de $\tilde{\varrho}$ s'en déduisent par l'homomorphisme de $\tilde{T}$ sur $T$ induit par $\tilde{\varphi}$. L'application du lemme 5 du Chapitre $I, n^{\circ} 12$, se réduit alors à un calcul qui ne présente aucune difficulté, et donne le résultat annoncé. La vérification se fait de même dans le cas (II).

Comme on a $m \geqslant 2 r$, d'où $m-r \geqslant m / 2$, dans le cas (I), on voit que la condition (B) du Chapitre III, $\mathrm{n}^{\circ} 38$ (c'est-à-dire $m>2 n+4 \varepsilon-2$ dans le cas (I) et $m>p+q$ dans le cas (II)) entraîne toujours la condition de convergence de la proposition 8 , dans le cas (I) comme dans le cas (II); elle lui est même équivalente, dans le cas (I), si $m=2 r$, et, dans le cas (II), si $m \neq 1$.

52. Nous pouvons maintenant démontrer la « formule de Siegel »; elle est contenue dans le théorème suivant :

THÉoR̀̀m 5. Soient $\mathcal{A}_{k}$ une algèbre de type $(\mathrm{I})$ et $X_{k}$ un $\mathcal{A}_{k}$-module à gauche, satis-

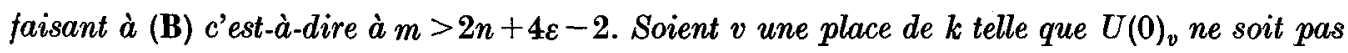
vide, et $G_{v}^{\prime}$ un sous-groupe de $G_{v}$ qui opère transitivement sur $U(i)_{v}$ quel que soit $i \in I(X)_{k}$. 
Soit $\nu$ une mesure positive sur $G_{A} / G_{k}$, invariante par $G_{v}^{\prime}$, telle que $\nu\left(G_{A} / G_{k}\right)=1$ et que l'intégrale

$$
I_{v}(\Phi)=\int_{G_{A} / G_{k}} \sum_{\xi \in X_{k}} \Phi(\varrho(u) \xi) \cdot d v(u)
$$

soit absolument convergente quelle que soit $\Phi \in S\left(X_{A}\right)$. Alors on a $I_{v}(\Phi)=E(\Phi)$, et, pour tout $i \in I(X)_{k}$ :

$$
\int_{G_{A} / G_{k}} \sum_{\xi \in U(i)_{k}} \Phi(\varrho(u) \xi) \cdot d \nu(u)=\int \Phi d \mu_{i}
$$

où $\mu_{i}$ est la mesure $\left|\theta_{i}\right|_{A}$ déterminée sur $U(i)_{A}$ par la jauge $\theta_{i}$ définie au théorème 2 du Chapitre $\mathrm{IV}, n^{\circ} 44$. Ces résultats restent valables quand on y remplace $G$ et $\varrho$ par $G_{1}$ et $\varrho_{1}$, ou bien, dans les cas $\left(\mathrm{I}_{3}\right),\left(\mathrm{I}_{4}\right)$, par $\tilde{G}$ et $\tilde{\varrho}$. Ils sont valables en particulier quand on prend pour $v$ la mesure de Haar sur $G$ normée par $\nu\left(G_{A} / G_{k}\right)=1$, ou encore la mesure de Haar sur $G_{1}$ (ou, dans les cas $\left(\mathrm{I}_{3}\right),\left(\mathrm{I}_{4}\right)$, sur $\left.\widetilde{G}\right)$ normée de même.

On procédera par récurrence sur le rang $n$ de $X_{k}$; pour cela, on va montrer d'abord que les hypothèses faites sur $v$ et $v$ relativement à $X_{k}$ entraînent que $v$ et $v$ ont les propriétés analogues relativement à tout $\mathcal{A}_{k}$-module $X_{k}^{\prime}$ de rang $n^{\prime} \leqslant n$. Tous les $\mathcal{A}_{k}$-modules de même rang étant isomorphes, il suffit de démontrer cette assertion quand $X_{k}^{\prime}$ est un sous-module de $X_{k}$. En ce qui concerne les conditions imposées à $v$, l'assertion est évidente. En ce qui concerne $v$, convenons, pour tout $i^{\prime} \in I\left(X^{\prime}\right)_{k}$, de désigner par $U^{\prime}\left(i^{\prime}\right)_{v}$ l'ensemble des éléments $x^{\prime}$ de $X_{v}^{\prime}$, de rang maximal dans $X_{v}^{\prime}$, qui satisfont $\grave{a} i_{X^{\prime}}\left(x^{\prime}\right)=i^{\prime}$. Considérons d'abord l'hypothèse $U(0)_{v} \neq \varnothing$, dont nous voulons faire voir qu'elle entraîne $U^{\prime}(0)_{v} \neq \varnothing$. Reprenons les notations du $\mathrm{n}^{\circ} 26 \mathrm{du}$ Chapitre II, en y prenant $K=k_{v}$ et écrivant $m_{v}, n_{v}$ au lieu de $m_{K}, n_{K}$. Supposons d'abord que $\mathcal{A}_{v}$ soit de type (I); comme au $\mathrm{n}^{\circ} 26 \mathrm{du}$ Chapitre II, écrivons $\mathcal{A}_{v}$ sous la forme $M_{m_{v}}(\mathfrak{K})$, où $\mathfrak{\Re}$ est une algèbre à division sur $k_{v}$ et où $m_{v}=m v$; l'involution $\iota$ sur $\mathcal{A}_{v}$ est alors définie par une involution sur $\mathfrak{K}$, et par une matrice $h_{v}$ à $m_{v}$ lignes et $m_{v}$ colonnes sur $\Re, \eta_{v}$-hermitienne par rapport à cette involution. Dire que $U(0)_{v} \neq \varnothing$ revient alors à dire que $h_{v}$ est d'indice $\geqslant n_{v}=n \nu$, et $U^{\prime}(0)_{v} \neq \varnothing$ revient de même à dire que cet indice est $\geqslant n_{v}^{\prime}=n^{\prime} v$; pour $n^{\prime} \leqslant n$, la première assertion entraîne évidemment la seconde. Si $\mathcal{A}_{v}$ est de type (II), $U(0)_{v} \neq \varnothing$ et $U^{\prime}(0)_{v} \neq \varnothing$ sont respectivement équivalentes à $m_{v} \geqslant 2 n_{v}$ et à $m_{v} \geqslant 2 n_{v}^{\prime}$, d'après le $\mathrm{n}^{\circ} 23 \mathrm{du}$ Chapitre II, et on en tire la même conclusion; on pourra remarquer d'ailleurs que, dans ce cas, on a $\varepsilon=\frac{1}{2}$ d'après le $n^{\circ} 26$ du Chapitre II, donc $m>2 n$ d'après $(\mathbf{B})$, de sorte qu'on a certainement $U(0)_{v} \neq \varnothing$ et $U^{\prime}(0)_{v} \neq \varnothing$. Passons à la transitivité de $G_{v}^{\prime}$ sur les ensembles $U(i)_{v}, U^{\prime}\left(i^{\prime}\right)_{v}$. Identifions $X_{k}$, comme d'habitude, avec $M_{m, n}(\mathfrak{f})$, et $X_{k}^{\prime}$ avec le module des éléments de $M_{m, n}(\mathfrak{f})$ de la forme $\left(x_{1}, \ldots, x_{n^{\prime}}, 0, \ldots, 0\right)$; désignons par $X_{k}^{\prime \prime}$ le module des éléments de $M_{m, n}(\mathfrak{l})$ de la forme $\left(0, \ldots, 0, x_{n^{\prime}+1}, \ldots, x_{n}\right)$, 
de sorte qu'on a $X_{k}=X_{k}^{\prime} \oplus X_{k}^{\prime \prime}$. Identifions $I\left(X_{k}\right)$ et $I\left(X_{k}^{\prime}\right)$ avec les espaces de matrices $\eta$-hermitiennes sur $\mathfrak{l}$, d'ordre $n$ et d'ordre $n^{\prime}$, respectivement, comme il a été expliqué au $\mathrm{n}^{\mathrm{o}} 19 \mathrm{du}$ Chapitre II. Soit $i^{\prime} \in I\left(X_{k}^{\prime}\right)$, et soit $i$ l'élément de $I\left(X_{k}\right)$ donné par la matrice $\left(\begin{array}{ll}i^{\prime} & 0 \\ 0 & 0\end{array}\right)$ à $n$ lignes et $n$ colonnes. Supposons d'abord que $\mathcal{A}_{v}$ soit de type (I); avec les mêmes notations que ci-dessus, on peut identifier $I\left(X_{v}\right)$ avec l'espace des matrices $\eta_{v}$-hermitiennes à $n_{v}$ lignes et $n_{v}$ colonnes sur $\mathfrak{K}$, et faire de même pour $I\left(X_{v}^{\prime}\right)$. L'isomorphisme canonique de $I\left(X_{k}\right) \otimes k_{v}$ sur $I\left(X_{v}\right)$, dont la définition résulte du $\mathrm{n}^{\circ} 15 \mathrm{du}$ Chapitre II, induit sur $I\left(X_{k}\right)$ une application $k$-linéaire de $I\left(X_{k}\right)$ dans $I\left(X_{v}\right)$; il serait aisé d'expliciter celle-ci, en introduisant quelques notations supplémentaires, mais cela est inutile pour notre objet. Soit $i_{v}$ l'élément de $I\left(X_{v}\right)$, image de $i$ par cette application; si $i_{v}^{\prime}$ est l'élément de $I\left(X_{v}^{\prime}\right)$ déduit de même de $i^{\prime}$, on aura $i_{v}=\left(\begin{array}{ll}i_{v}^{\prime} & 0 \\ 0 & 0\end{array}\right)$. D'après le $\mathrm{n}^{\circ} 19 \mathrm{du}$ Chapitre II, $U(i)_{v}$ s'identifie à l'ensemble des matrices $x$ à $m_{v}$ lignes et $n_{v}$ colonnes sur $\mathfrak{R}$, de rang maximal (c'est-à-dire égal à $\left.n_{v}\right)$, qui satisfont à $h_{v}[x]=i_{v}$; on a une assertion analogue pour $U^{\prime}\left(i^{\prime}\right)_{v}$. Par hypothèse, $U(0)_{v}$ n'est pas vide, ce qui veut dire que $h_{v}$ est d'indice $\geqslant n_{v}$; on en déduit aisément, par un raisonnement élémentaire, que $U(i)_{v}$ n'est pas vide; soit $a \in U(i)_{v}$. Alors, si $a^{\prime}$, $a^{\prime \prime}$ sont les projections de $a$ sur $X_{v}^{\prime}, X_{v}^{\prime \prime}$ pour la décomposition $X_{v}=X_{v}^{\prime} \oplus X_{v}^{\prime \prime}$ de $X_{v}$ en somme directe, on aura

$$
h_{v}\left[\left(a^{\prime}, a^{\prime \prime}\right)\right]=i_{v}=\left(\begin{array}{cc}
i_{v}^{\prime} & 0 \\
0 & 0
\end{array}\right)
$$

donc $h_{v}\left[a^{\prime}\right]=i_{v}^{\prime}$; de plus, comme $a$ est de rang maximal (égal à $n_{v}$ ) dans $X_{v}, a^{\prime}$ doit être de rang maximal (égal à $n_{v}^{\prime}$ ) dans $X_{v}^{\prime}$; on a donc $a^{\prime} \in U^{\prime}\left(i^{\prime}\right)_{v}$. Soit alors $x^{\prime} \in U^{\prime}\left(i^{\prime}\right)_{v}$. D'après la proposition $3 \mathrm{du}$ Chapitre II, $\mathrm{n}^{\circ} 22$, il y a $u \in G_{v}$ tel que $x^{\prime}=u a^{\prime}$. Alors $a$ et $u a$ appartiennent tous deux à $U(i)_{v}$, de sorte que, par hypothèse, il y a $u^{\prime} \in G_{v}^{\prime}$ tel que $u a=u^{\prime} a$, d'où $x^{\prime}=u^{\prime} a^{\prime}$. Cela montre bien que $G_{v}^{\prime}$ opère transitivement sur $U^{\prime}\left(i^{\prime}\right)_{v}$. La démonstration șe fait d'une manière tout à fait analogue lorsque $\mathcal{A}_{v}$ est de type (II).

Soient alors $v$ et $v$ satisfaisant aux hypothèses du théorème 5 relativement à un module $X_{k}$ de rang $n$, et considérons d'abord le cas de l'intégrale $I_{\nu}$ formée au moyen de $G$ et de $\varrho$. Pour $n=0$, les assertions du théorème se réduisent à $I_{v}=\delta_{0}$, ce qui est une conséquence évidente de l'hypothèse $v\left(G_{A} / G_{k}\right)=1$. Procédons par récurrence sur $n$, et supposons $n \geqslant 1$. Comme $I_{\nu}(\Phi)$ est convergente par hypothèse quelle que soit $\Phi \in S\left(X_{A}\right)$, le lemme 2 du Chapitre $\mathrm{I}, \mathrm{n}^{\circ} 2$, joint au lemme 5 de [14], $\mathrm{n}^{\circ} 41$, montre aussitôt que $I_{\nu}$ est une mesure tempérée positive. Le théorème 6 de [14], $n^{\circ} 41$, et la proposition 9 de [14], $n^{\circ} 51$, montrent alors que $I_{v}$ est invariante par $P s_{k}$; elle l'est évidemment aussi par $G_{v}^{\prime}$. Pour les mêmes raisons, si on note $I_{v_{i} i}(\Phi)$ le premier membre de $(41), I_{v, i}$ est une mesure tempérée positive. 
Soit $I_{v, X}$ la somme des $I_{v, i}$ pour $i \in I(X)_{k}$; on peut considérer $I_{v, X}$ comme défini par l'intégrale analogue à celle qui définit $I_{v}$, mais où la sommation est restreinte aux éléments $\xi$ de $X_{k}$ qui sont de rang maximal dans $X_{k}$. De même, pour tout sous-module $Z_{k}$ de $X_{k}$, désignons par $I_{y, z}$ la mesure tempérée positive définie par l'intégrale analogue à celle qui définit $I_{v}$, mais où la sommation est restreinte aux éléments $\xi$ de $Z_{k}$ qui sont de rang maximal dans $Z_{k}$, ou autrement dit aux éléments $\xi$ de $X_{k}$ qui satisfont à $\mathcal{A}_{k} \cdot \xi=Z_{k}$. Tenant compte du théorème $2 \mathrm{du}$ Chapitre IV, $\mathrm{n}^{\circ} 44$, on voit que le théorème 5 pour $X_{k}$ implique que $I_{\nu, X}=E_{X}$; par suite, l'hypothèse de récurrence implique que $I_{v, Z}=E_{Z}$ pour tout sous. module $Z_{k}$ de $X_{k}$, autre que $X_{k}$. On a donc, en vertu de cette hypothèse :

$$
I_{v}=\sum_{i \in I(X)_{k}} I_{v_{, i}}+\sum_{Z_{k} \neq X_{k}} E_{Z}
$$

D'après le théorème $3 \mathrm{du}$ Chapitre $\mathrm{IV}, \mathrm{n}^{\circ} 44$, la seconde somme du second membre n'est pas autre chose que $E-E_{X}$. D'autre part, d'après la proposition 3 du Chapitre II, $\mathrm{n}^{\circ} 22$, ceux des $U(i)_{k}$ qui ne sont pas vides sont des orbites de $G_{k}$ dans $X_{k}$; compte tenu du changement de notation, la formule (11) du Chapitre $I, n^{0} 7$, montre alors que les mesures $I_{v, i}$ sont respectivement portées par les $U(i)_{A}$, au sens où ce terme a été défini au $\mathrm{n}^{\circ} 49 \mathrm{du}$ Chapitre V. Par conséquent, $I_{p}$ satisfait à toutes les hypothèses du théorème 4 du Chapitre $\mathrm{V}, \mathrm{n}^{\circ} 50$. D'après ce théorème, on a donc $I_{\nu}=E$, et par suite $I_{v, X}=E_{X}$ d'après (42). Comme $I_{\nu_{1} i}$ et $\mu_{i}$ sont les restrictions de $I_{v_{,}}$et de $E_{X}$, respectivement, à l'ensemble $i_{X}^{-1}(\{i\})$, il s'ensuit qu'on a $I_{v, i}=\mu_{i}$ quel que soit $i \in I(X)_{k}$. Pour voir que le résultat ainsi obtenu s'applique quand on prend pour $\nu$ la mesure de Haar sur $G_{A}$, il suffit de prendre $G_{v}^{\prime}=G_{v}$, la place $v$ étant choisie de manière que $U(0)_{v}$ ne soit pas vide; or il en est ainsi pour presque tout $v$, par exemple d'après les résultats rappelés au $\mathrm{n}^{\circ} 4 \mathrm{du}$ Chapitre $\mathrm{I}$. Cela achève la démonstration en ce qui concerne $G$ et $\varrho$.

Il n'y aurait rien à changer à ce qui précède, en ce qui concerne $G_{1}$ et $\varrho_{1}$ (resp. $\widetilde{G}$ et Ø) si on était assuré, dans tous les cas, que $\left(G_{1}\right)_{K}$, opérant sur $X_{K}$ au moyen de $\varrho_{1}$ (resp. $\tilde{G}_{K}$ opérant au moyen de $\left.\tilde{\varrho}\right)$, eût les mêmes orbites que $G_{K}$ dans $X_{K}$, quel que soit le corps $K \supset k$; mais cette question ne semble pas toujours facile à trancher, et on peut se contenter de résultats partiels à cet égard. La question ne se pose pas si $\mathcal{A}_{k}$ est de type $\left(I_{0}\right)$ ou $\left(I_{1}\right)$, puisqu'alors $\tilde{G}$ n'est pas défini et $G_{1}=G$. Si $\mathcal{A}_{K}$ est de type (II), $\mathcal{A}_{k}$ doit être de type $\left(\mathrm{I}_{2}\right)$ d'après le $\mathbf{n}^{\circ} 26$ du Chapitre II, de sorte qu'on a $\varepsilon=\frac{1}{2}$, donc $m>2 n$ d'après $(\mathbf{B})$, d'où $m_{K}>$ $2 n_{K}$; au moyen des résultats du $n^{\circ} 23 \mathrm{du}$ Chapitre II, on vérifie alors facilement que $\left(G_{1}\right)_{K}$ opère transitivement sur les orbites de $G_{K}$ dans $X_{K}$. Il en sera ainsi, en particulier, si $A_{k}$ est de type $\left(I_{2}\right)$ et qu'on prenne pour $K$ le domaine universel. Quand $\mathcal{A}_{K}$ est de type $\left(\mathrm{I}_{4}\right)$, on vérifie de même que $\left(G_{1}\right)_{K}$ opère transitivement sur les orbites de $G_{K}$ dans $X_{K}$ pourvu que $m_{K}>2 n_{K} ;(\mathbf{B})$ étant toujours supposée satisfaite, il en sera ainsi chaque fois que $\mathcal{A}_{k}$ 
A. WEIL

est de type $\left(I_{3}\right)$ ou $\left(I_{4}\right)$ et que $K$ est le domaine universel. Enfin, l'homomorphisme $\tilde{\varphi}_{1}$ $\operatorname{de} \widetilde{G}$ sur $G_{1}$ détermine un homomorphisme de $\widetilde{G}_{K}$ sur $\left(G_{1}\right)_{K}$ chaque fois que $K$ est algébriquement clos; donc $\widetilde{G}_{K}$ opère alors transitivement sur les orbites de $\left(G_{1}\right)_{K}$ dans $X_{K}$, et il en est ainsi en particulier quand $K$ est le domaine universel. Cela posé, si on reprend la démonstration donnée ci-dessus de la première partie du théorème 5 pour $G$, on voit qu'elle s'applique aussi à $G_{1}$ (resp. à $\widetilde{G}$ ) pourvu qu'on sache que les $I_{v, i}$ sont des mesures respectivement portées par les $U(i)_{A}$. Or $U(i)_{k}$ est en tout cas une réunion d'orbites de $\left(G_{1}\right)_{k}$ (resp. $\left.\widetilde{G}_{k}\right)$, à chacune desquelles on peut appliquer la formule (11) du Chapitre I, $n^{0} 7$; comme, dans cette formule, $H(\omega)$ désigne l'orbite de $\xi_{\omega}$ sur le domaine universel, on voit qu'il suffit ici de savoir que $U(i)$ est l'orbite de tout point de $U(i)_{k}$ par $G_{1}$ (resp. $\widetilde{G}$ ) sur le domaine universel; or cela résulte de ce qui précède.

Il ne reste plus qu'à vérifier la dernière assertion du théorème 5 en ce qui concerne $G_{1}$ (resp. $\tilde{G}$ ); pour cela, il suffit de faire voir qu'on peut choisir $v$ de manière que $U(0)_{v}$ ne soit pas vide et que $\left(G_{1}\right)_{v}$ (resp. $\widetilde{G}_{v}$ ) opère transitivement sur les $U(i)_{v}$. Si $\mathcal{A}_{k}$ est de type $\left(\mathrm{I}_{0}\right)$ ou $\left(\mathrm{I}_{1}\right)$, il n'y a rien à démontrer. Si $\mathcal{A}_{k}$ est de type $\left(\mathrm{I}_{2}\right)$, il suffit, d'après ce qui précède et d'après le $\mathrm{n}^{\circ} 23 \mathrm{du}$ Chapitre II, de choisir $v$ de manière que $\mathcal{A}_{v}$ soit de type (II). Soit $\mathcal{A}_{k}$ de type $\left(\mathrm{I}_{3}\right)$ ou $\left(\mathrm{I}_{4}\right)$; pour presque tout $v, U(0)_{v}$ est non vide et $\mathcal{A}_{v}$ est de type $\left(\mathrm{I}_{4}\right)$, et par suite, d'après ce qui précède, $G_{v}^{\prime}=\left(G_{1}\right)_{v}$ aura les propriétés voulues. En ce qui concerne $\widetilde{G}_{v}$, choisissons $v$ comme on vient de le dire, en supposant de plus que $v$ ne soit pas une place à l'infini. La condition (B) implique alors $m_{v}-2 n_{v} \geqslant 3$; en faisant usage des résultats du $\mathrm{n}^{\circ} 23$ du Chapitre II et des propriétés connues de la " norme spinorielle ", on conclut aisément qu'alors $\widetilde{G}_{v}$ opère transitivement sur les orbites de $\left(G_{1}\right)_{v}$ dans $X_{v}$. Cela achève la démonstration du théorème 5 .

Quand on prend pour $v$ la mesure de Haar dans $G_{A}$, la formule $I_{v}=E$ est celle même qui a été annoncée dans le mémoire précédent ([14], $\left.\mathrm{n}^{\circ} 52\right)$.

53. Appliquant maintenant le théorème $5 \mathrm{du} \mathrm{n}^{\circ} 52$ au groupe $G$, et à la mesure de Haar $v$ sur $G$, nous allons dégager de la formule (41) quelques résultats arithmétiques qui s'y trouvent renfermés. Le premier, de nature qualitative, s'obtient en observant que la mesure tempérée définie par le premier membre de (41) est 0 chaque fois que $U(i)_{k}$ est vide, alors que la mesure $\mu_{i}$ qui figure au second membre ne peut être 0 , en vertu de sa définition, que si $U(i)_{A}$ est vide. Par suite, quand (B) est satisfaite, les assertions $U(i)_{k} \neq \emptyset$ et $U(i)_{A} \neq \emptyset$ sont équivalentes. Compte tenu des résultats mentionnés au $\mathrm{n}^{\circ} 4 \mathrm{du}$ Chapitre $I$, il revient au même de dire que, si (B) est satisfaite, $U(i)_{k} \neq \varnothing$ équivaut à l'assertion « $U(i)_{v} \neq \varnothing$ quel que soit $v$ ". Sous cette forme, on reconnaît le " principe de Hasse ", à cela près que ce principe, comme on sait, reste valable même pour certaines valeurs de $m$ et $n$ qui ne satisfont 
pas à (B); par exemple, si $\mathcal{A}_{k}$ est de type $\left(\mathbf{I}_{4}\right)$, il est valable quels que soient $m$ et $n$. En revanche, les remarques de la fin du Chapitre III, $\mathrm{n}^{\circ} 38$, jointes à ce qu'on vient de démontrer, font voir que, si (B) est satisfaite, $U(i)_{k} \neq \varnothing$ équivaut même à l'assertion " $U(i)_{v} \neq \varnothing$ quelle que soit la place à l'infini $v$ de $k$ ". Par exemple, pour $k=\mathbf{Q}, \mathcal{A}_{k}$ de type $\left(\mathrm{I}_{4}\right), n=1$ et $i=0$, on obtient ainsi le thèorème classique de Meyer, d'après lequel toute forme quadratique indéfinie à $m \geqslant 5$ variables, sur $\mathbf{Q}$, " représente 0 ».

De (41), on va tirer maintenant des résultats quantitatifs concernant les " nombres de Tamagawa " des groupes classiques. Observons d'abord qu'en vertu de la proposition $3 \mathrm{du}$ Chapitre II, $\mathrm{n}^{\circ} 22$, et de son corollaire, la condition (W) du $\mathrm{n}^{\circ} 8 \mathrm{du}$ Chapitre I est bien satisfaite par $G$ opérant sur $X$ au moyen de la représentation $\varrho$, de sorte qu'on peut appliquer les résultats du $\mathrm{n}^{\circ} 8 \mathrm{du}$ Chapitre I au premier membre de (41). Soit donc $\xi$ un élément de rang maximal de $X_{k}$; soit $i=i_{X}(\xi)$, et soit $g$ le groupe de stabilité de $\xi$ dans $G$; d'après le $\mathrm{n}^{\circ} 8 \mathrm{du}$ Chapitre $\mathrm{I}$, on peut identifier $U(i)$ avec $G / g$ et $U(i)_{A}$ avec $G_{A} / g_{A}$; de plus, d'après ce $\mathrm{n}^{\circ}, g_{A} / g_{k}$ est de mesure finie, ce qu'il est d'ailleurs facile de vérifier directement au moyen des résultats du $n^{\circ} 23$ du Chapitre II qui donnent la structure de $g$. Soit $v_{0}$ la mesure de Haar sur $g$, normée par la condition $v_{0}\left(g_{A} / g_{k}\right)=1$, Le $\mathrm{n}^{\circ} 8 \mathrm{du}$ Chapitre $\mathrm{I}$ montre alors que la mesure $I_{\nu, i}$ définie par le premier membre de (41) n'est autre que la mesure $v / v_{0}$ portée par $G_{A} / g_{A}=U(i)_{A} ;(41)$ peut donc s'exprimer en disant que $v / v_{0}=\left|\theta_{i}\right|_{A}$.

Soient $d u, d v$ des jauges invariantes sur $G$ et sur $g$, respectivement; on en déduit, comme on sait (cf. [13], Th. 2.4.1) une jauge invariante $d u / d v$ sur $U(i)$, qui doit donc coïncider avec $\theta_{i}$ à un facteur scalaire près; par suite, en multipliant au besoin $d u$ par un tel facteur, on peut faire en sorte qu'on ait $d u / d v=\theta_{i}$. Soit $\lambda$ un système de facteurs de convergence pour $G$; alors, d'après un résultat général ([13], Theorem 2.4.3) joint au lemme $19 \mathrm{du}$ Chapitre $\mathrm{IV}, \mathrm{n}^{\circ} 43, \lambda$ est aussi un système de facteurs de convergence pour $g$, et on a

$$
\left|\theta_{i}\right|_{A}=|\lambda d u|_{A} /|\lambda d v|_{A} .
$$

Chaque fois que $\lambda$ est un système de facteurs de convergence pour un groupe $G$, on conviendra de noter $\tau_{\lambda}(G)$ la mesure de $G_{A} / G_{k}$ pour la mesure de Haar $|\lambda d u|_{A}$ sur $G_{A}, d u$ étant une jauge invariante sur $G$; pour $\lambda=1, \tau_{\lambda}(G)$ est par définition le " nombre de Tamagawa " de $G$. Avec cette notation, les mesures $\nu, v_{0}$ définies plus haut, sur $G_{A}$ et sur $g_{A}$ respectivement, peuvent s'écrire :

$$
d \nu(u)=\tau_{\lambda}(G)^{-1}|\lambda d u|_{A}, \quad d v_{0}(v)=\tau_{\lambda}(g)^{-1}|\lambda d v|_{A} .
$$

Tenant compte de ce qui précède, on voit que (41) équivaut à la formule

$$
\tau_{\lambda}(g)=\tau_{\lambda}(G),
$$

qui est donc valable chaque fois que $(B)$ est satisfaite et que $g$ est le groupe de stabilité dans $G$ d'un point $\xi$ de $X_{k}$ de rang maximal dans $X_{k}$. 6-652922. Acta mathematica. 113. Imprimé le 11 mars 1965. 
54. Plaçons-nous d'abord dans le cas $\left(\mathrm{I}_{0}\right)$; alors $G$ est le groupe symplectique $S p(m)$ à $m$ variables, $m$ étant nécessairement pair. On peut alors prendre $\lambda=1$. Prenons $n=1$; alors on a $I(X)_{k}=\{0\}$, et le $\mathrm{n}^{\circ} 23$ du Chapitre II fait voir que $g$ est isomorphe à un produit semidirect de $S p(m-2)$ et d'un groupe unipotent. Par suite, (43) montre, pour $m>2$, que $S p(m)$ a même nombre de Tamagawa que $S p(m-2)$, et, pour $m=2$, que ce nombre a la valeur 1 .

Si $\mathcal{A}_{k}$ n'est pas de type $\left(\mathrm{I}_{0}\right)$, on peut, par le choix d'une base convenable, faire en sorte que la forme $\eta$-hermitienne $h$ soit donnée par une matrice diagonale; soient $a_{1}, \ldots, a_{m}$ les éléments diagonaux de celle-ci; convenons d'écrire alors $G\left(a_{1}, \ldots, a_{m}\right)$ au lieu de $G$, et $\tau_{\lambda}\left(a_{1}, \ldots, a_{m}\right)$ au lieu de $\tau_{\lambda}(G)$. Prenons $n=1, X_{k}$ s'identifiant alors à $M_{m .1}(\mathfrak{f})$, et appliquons (43) au groupe de stabilité $g$ de $\xi=(0, \ldots, 0,1)$. Soit d'après le $n^{\circ} 23$ du Chapitre II, soit directement, on voit aussitôt que $g$ est isomorphe à $G\left(a_{1}, \ldots, a_{m-1}\right)$; on a donc

$$
\tau_{\lambda}\left(a_{1}, \ldots, a_{m-1}\right)=\tau_{\lambda}\left(a_{1}, \ldots, a_{m}\right)
$$

chaque fois que $m>4 \varepsilon$. De même, appliquons (43) au cas où on a pris

$$
G=G\left(a_{1}, \ldots, a_{m-2}, 1,-1\right), \quad \xi=(0, \ldots, 0,1,1)
$$

comme on a alors $i_{X}(\xi)=0$, le $\mathrm{n}^{\circ} \mathbf{2 3} \mathrm{du}$ Chapitre II montre que $g$ est isomorphe à un produit semidirect de $G\left(a_{1}, \ldots, a_{m-2}\right)$ et d'un groupe unipotent; on a donc

$$
\tau_{\lambda}\left(a_{1}, \ldots, a_{m-2}\right)=\tau_{\lambda}\left(a_{1}, \ldots, a_{m-2}, 1,-1\right)
$$

chaque fois que $m>4 \varepsilon$, à condition de convenir que le premier membre a la valeur 1 pour $m=2$.

Dans le cas $\left(\mathrm{I}_{1}\right)$, on peut prendre $\lambda=1$, et il résulte de ce qui précède que le nombre de Tamagawa de $G$ est toujours 1 . Dans les cas $\left(\mathrm{I}_{2}\right),\left(\mathrm{I}_{3}\right),\left(\mathrm{I}_{4}\right)$, on peut seulement en conclure que $\tau_{\lambda}\left(a_{1}, \ldots, a_{m}\right)$ est indépendant des $a_{\mu}$ et de $m$ pourvu que $m>4 \varepsilon-2$. Ce résultat est nouveau, à ce qu'il semble, dans le cas $\left(I_{3}\right)$, et aussi dans le cas $\left(I_{2}\right)$ si $\mathfrak{l}$ n’est pas commutatif.

Rappelons qu'on peut déterminer $\tau_{\lambda}(G)$ directement, au moyen des « isomorphismes canoniques ", pour $m=3$ dans le cas $\left(\mathrm{I}_{4}\right)$, pour $m=2$ dans le cas $\left(\mathrm{I}_{3}\right)$, et aussi pour $m=1$ dans le cas $\left(\mathrm{I}_{2}\right)$ lorsque $\mathfrak{f}$ est commutatif ou bien est une algèbre de quaternions sur son centre. Par suite, dans tous ces cas, on obtient ainsi, en substance, la détermination complète des nombres de Tamagawa.

Enfin, quand les groupes $G_{1}$ et $\tilde{G}$, introduits plus haut, satisfont à la condition (W) du Chapitre I, $n^{\circ} 8$, on peut leur appliquer la même méthode, et en déduire des conséquences analogues. 
55. Désignons par $\mathcal{G}$ l'un des groupes considérés ci-dessus, c'est-à dire $G, G_{1}$ ou $\tilde{G}$. Soit $\mathcal{G}^{\prime}$ un sous-groupe ouvert de $\mathcal{G}_{A}$, et soit $\mathcal{G}_{k}^{\prime}=\mathcal{G}_{k} \cap \mathcal{G}^{\prime}$. On peut, d'une manière évidente, identifier $\mathcal{G}^{\prime} / \mathcal{G}_{k}^{\prime}$ avec $\mathcal{G}^{\prime} \mathcal{G}_{k} / \mathcal{G}_{k}$, c'est-à-dire avec l'image de $\mathcal{G}^{\prime}$ dans $\mathcal{G}_{A} / \mathcal{G}_{k}$; celle-ci est une partie ouverte et fermée de $\mathcal{G}_{A} / \mathcal{G}_{k}$. Si $\nu$ désigne de nouveau la mesure de Haar dans $\mathcal{G}_{A}$, normée par $v\left(\mathcal{G}_{A} / \mathcal{G}_{k}\right)=1$, on aura done :

$$
0<\nu\left(\mathcal{G}^{\prime} \mathcal{G}_{k} / \mathcal{G}_{k}\right) \leqslant 1
$$

Posons $\alpha=\boldsymbol{\nu}\left(\mathcal{G}^{\prime} \mathcal{G}_{k} / \mathcal{G}_{k}\right)$, et soit $f$ la fonction caractéristique de l'ensemble $\mathcal{G}^{\prime} \mathcal{G}_{k}$ dans $\mathcal{G}_{\mathrm{A}}$. Dans $\mathcal{G}_{A}$, considérons la mesure $v_{1}$ donnée par

$$
d \nu_{1}(u)=\alpha^{-1} f(u) d v(u)
$$

Cette mesure est invariante à droite par $\mathcal{G}_{k}$ et détermine, par passage au quotient, une mesure sur $\mathcal{G}_{A} / \mathcal{G}_{k}$, qu'on notera encore $\nu_{1}$; celle-ci induit sur $\mathcal{G}^{\prime} \mathcal{G}_{k} / \mathcal{G}_{k}$, ou, ce qui revient au même, sur $\mathcal{G}^{\prime} / \mathcal{G}_{k}^{\prime}$, une mesure $\nu^{\prime}$, invariante à gauche par $\mathcal{G}^{\prime}$ et satisfaisant à $\nu^{\prime}\left(\mathcal{G}^{\prime} / \mathcal{G}_{k}^{\prime}\right)=1$; il est clair que $\nu^{\prime}$ est complètement déterminée par ces dernières conditions.

Désignons par $\mathfrak{J}(\Phi)$ l'intégrale analogue à $I_{\nu}(\Phi)$, où $G$ est remplacé par $\mathcal{G}$ (et $\varrho$ par $\varrho, \varrho_{1}$ ou $\varrho$ suivant les cas); d'après le théorème 5 , on a $\mathfrak{J}=E$. Désignons par $\mathcal{J}^{\prime}(\Phi)$ l'intégrale analogue prise au moyen de $\nu^{\prime}$ sur $\mathcal{G}^{\prime} / \mathcal{G}_{k}^{\prime}$, $\varrho$ étant remplacée par la représentation de $\mathcal{G}^{\prime}$ dans $X_{A}$ induite par $\varrho, \varrho_{1}$ ou $\tilde{\varrho}$. On peut aussi considérer $\mathcal{I}^{\prime}(\Phi)$ comme étant l'intégrale analogue à $I_{\nu}(\Phi)$ prise sur $\mathcal{G}^{\prime} \mathcal{G}_{k} / \mathcal{G}_{k}$ au moyen de $v^{\prime}$, ou, ce qui revient au même, prise sur $\mathcal{G}_{A} / \mathcal{G}_{k}$ au moyen de $v_{1}$. Cette dernière définition fait voir que, pour $\Phi \geqslant 0, \mathcal{J}^{\prime}(\Phi)$ est majorée par $\alpha^{-1} \mathcal{J}(\Phi)$, donc que cette intégrale est absolument convergente quelle que soit $\Phi \in \boldsymbol{S}\left(X_{A}\right)$.

Les travaux de Siegel sur les formes quadratiques indéfinies conduisent à se demander à quelles conditions on a $\mathfrak{I}^{\prime}=E$. D'après le théorème 5 , il suffit pour cela qu'il y ait une place $v$ de $k$ telle que $U(0)_{v}$ ne soit pas vide et que la mesure $v_{1}$, ou, ce qui revient au même, l'ensemble $\mathcal{G}^{\prime} \mathcal{G}_{k}$, soient invariants à gauche par un sous-groupe de $\mathcal{G}_{v}$ qui opère transitivement sur $U(i)_{v}$ quel que soit $i \in I(X)_{k}$.

Il en sera évidemment ainsi s'il existe $v$ telle que $U(0)_{v}$ ne soit pas vide et que $\mathcal{G}_{v} \cap \mathcal{G}^{\prime}$ ait la propriété de transitivité en question. De plus, lorsqu'il en est ainsi, le théorème 5 montre que l'analogue de (4l) est valable pour $\mathfrak{J}^{\prime}$. Il s'ensuit en particulier que, dans ces conditions, le support de la mesure définie par le premier membre de (41) est $U(i)_{A}$; par suite, l'ensemble des éléments $\varrho(u) \xi$, pour $u \in \mathcal{G}^{\prime}, \xi \in U(i)_{k}$, est partout dense dans $U(i)_{A}$ quand les conditions précédentes sont satisfaites (y compris la condition $(\mathbf{B})$ ). C'est là le "théorème d'approximation » qui a été annoncé dans l'Introduction.

56. Il y aurait lieu de comparer ce dernier résultat avec les théorèmes d'approximation qui ont été obtenus dans ces dernières années par voie arithmétique, et principalement 
avec celui de M. Kneser ([8]) qui semble les comprendre tous comme cas particuliers. Nous n'entreprendrons pas cette comparaison ici; mais nous appliquerons le théorème de Kneser à la solution du problème de Siegel dans des cas où on ne peut raisonner comme on a fait plus haut.

Pour cela, supposons qu'on ait pris $\mathcal{G}^{\prime}$ de la forme $\prod \mathcal{G}_{v}^{\prime}$, où $\mathcal{G}_{v}^{\prime}$ est, pour tout $v$, un sous-groupe ouvert de $\mathcal{G}_{v}$, avec $\mathcal{G}_{v}^{\prime}=\mathcal{G}_{v}^{\circ}$ pour presque tout $v$; supposons de plus qu'il y ait une place $v$ pour laquelle on ait $\mathcal{G}_{v}^{\prime}=\mathcal{G}_{v}$ et pour laquelle $\mathcal{G}_{v}$ ne soit pas compact. On va voir qu'alors on a $\mathfrak{J}^{\prime}=E$.

Désignons par $\tilde{\mathcal{G}}$ le groupe $G$ lorsque $\mathcal{A}_{k}$ est de type $\left(\mathrm{I}_{0}\right)$ ou $\left(\mathrm{I}_{1}\right)$, le groupe $G_{1}$ lorsque $\mathcal{A}_{k}$ est de type $\left(\mathrm{I}_{2}\right)$, et le groupe $\tilde{G}$ lorsque $\mathcal{A}_{k}$ est de type $\left(\mathrm{I}_{3}\right)$ ou $\left(\mathrm{I}_{4}\right)$. Dans tous les cas, $\tilde{\mathcal{G}}$ est donc connexe et simplement connexe, et on peut lui appliquer le théorème de Kneser. En particulier, si $\mathcal{G}=\tilde{\mathcal{G}}$, celui-ci montre qu'avec les hypothèses ci-dessus on a $\mathcal{G}^{\prime} \mathcal{G}_{k}=\mathcal{G}_{A}$, donc $\mathfrak{J}^{\prime}=\mathfrak{J}$, d'où le résultat annoncé. Dans tout autre cas, soit $\psi$ l'homomorphisme de $\tilde{G}$ dans $\mathcal{G}$ défini au $n^{\circ} 51$ (c'est-à-dire $\varphi_{1}$ dans le cas $\left(I_{2}\right)$ si $\mathcal{G}=G, \tilde{\varphi}$ dans les cas $\left(\mathrm{I}_{3}\right),\left(\mathrm{I}_{4}\right)$ si $\mathcal{G}=G$, et $\tilde{\varphi}_{1}$ dans ces mêmes cas si $\mathcal{G}=G_{1}$ ); $\psi$ détermine un homomorphisme $\psi_{A}$ de $\tilde{\mathcal{G}_{A}}$ dans $\mathcal{G}_{A}$. Posons $\tilde{\mathcal{G}}^{\prime}=\psi_{A}^{-1}\left(\mathcal{G}^{\prime}\right)$; d'après le théorème de Kneser, on a $\tilde{\mathcal{G}}^{\prime} \tilde{\mathcal{G}}_{k}=\tilde{\mathcal{G}}_{A}$.

On va montrer qu'alors la mesure $\nu_{1}$ définie par (44) est invariante à gauche par $\tilde{\mathcal{G}}_{A}$ opérant sur $\mathcal{G}_{A}$ par $\psi_{A}$; pour cela, il suffit de faire voir qu'il en est ainsi de l'ensemble $\mathcal{G}^{\prime} \mathcal{G}_{k}$, c'est-à-dire qu'on a $\psi_{A}(g) x y \in \mathcal{G}^{\prime} \mathcal{G}_{k}$ quels que soient $g \in \tilde{\mathcal{G}}_{A}, x \in \mathcal{G}^{\prime}, y \in \mathcal{G}_{k}$. Or on vérifie facilement, dans chacun des cas qu'on a à envisager ici, que $\psi_{A}\left(\tilde{\mathcal{G}}_{A}\right)$ est un sous-groupe invariant de $\mathcal{G}_{A}$. Il s'ensuit que, si $g \in \tilde{\mathcal{G}}_{A}$ et $x \in \mathcal{G}_{A}, x^{-1} \psi_{A}(g) x$ appartient à $\psi_{A}\left(\tilde{\mathcal{G}}_{A}\right)$ et est donc de la forme $\psi_{A}(\tilde{x} \tilde{y})$ avec $\tilde{x} \in \tilde{\mathcal{G}}^{\prime}, \tilde{y} \in \tilde{\mathcal{G}}_{k}$ d'après le théorème de Kneser. On a donc

$$
\psi_{A}(g) x y=x \psi_{A}(\tilde{x}) \cdot \psi_{A}(\tilde{y}) y
$$

le second membre appartient bien à $\mathcal{G}^{\prime} \mathcal{G}_{k}$. On a ainsi montré que $\nu_{1}$ est invariante par $\tilde{\mathcal{G}}_{A}$, donc par $\tilde{\mathcal{G}}_{v}$ quel que soit $v$. Comme on a fait voir à la fin de la démonstration du théorème 5 qu'il existe toujours $v$ tel que $U(0)_{v}$ ne soit pas vide et que $\tilde{\mathcal{G}}_{v}$ opère transitivement sur les $U(i)_{v}$, le théorème 5 s'applique à $\boldsymbol{y}_{1}$, ce qui achève la démonstration.

On a ainsi retrouvé, quelque peu généralisés, tous les résultats démontrés par Siegel au cours de ses travaux sur les formes quadratiques, ainsi que ceux qui sont énoncés à la fin de [12], à l'exception des suivants. Tout d'abord, nous n'avons pas abordé l'étude du cas où $m=2 n+4 \varepsilon-2$; or les résultats obtenus par Siegel dans le cas où $\mathcal{A}_{k}$ est de type $\left(\mathrm{I}_{4}\right)$ sur $k=\mathbf{Q}$ ou $k=\mathbf{Q}(\sqrt{-\overline{1}})$, et où on prend $m=4, n=1$, donnent à penser que, si $m=2 n+4 \varepsilon-2$, la série d'Eisenstein-Siegel cesse d'être absolument convergente, mais qu'on peut néan- 
moins, lorsque la condition de convergence de la proposition 8 du $n^{\circ} 51$ est satisfaite, " sommer " la série d'Eisenstein-Siegel au moyen de "facteurs de sommation " convenablement choisis, et retrouver ainsi l'analogue de la formule de Siegel. Il resterait aussi à examiner, du point de vue qui a été le nôtre dans ce travail, les résultats de Siegel sur les fonctions zêta des formes quadratiques indéfinies, qui sont étroitement liés aux questions qu'on a étudiées ici.

57. Le cas où $\mathcal{A}_{k}$ est de type (II) ne semble pas se prêter pour l'instant à des résultats aussi généraux ou aussi simples que le cas (I). Cependant, si $\mathcal{A}_{k}$ est de type (II) et $X_{k}$ de rang $(1,1)$, on peut procéder comme suit. Comme toujours, supposons (B) satisfaite, ce qui revient ici à $m>2$. Reprenons l'intégrale $I_{1}(\Phi)$ qui figure dans la proposition 8 du $\mathrm{n}^{\circ} 51$; on va la décomposer suivant les orbites de $\left(G_{1}\right)_{k}$ dans $X_{k}$, par application des résultats des $\mathbf{n}^{\text {os }} 7-8 \mathrm{du}$ Chapitre $\mathrm{I}$.

Modifiant un peu nos notations habituelles, écrivons $X_{k}$ comme somme directe de deux modules $Y_{k}, Z_{k}$, de rangs respectifs $(1,0)$ et $(0,1)$; ceux-ci sont respectivement isomorphes à $M_{m, 1}(\mathfrak{f})$ et à $M_{m, 1}\left(\mathfrak{f}^{\prime}\right)$. Les groupes $G_{k},\left(G_{1}\right)_{k}$ s'idéntifient respectivement à $G L(m, \mathfrak{l})$ et à $S L(m, \mathfrak{l}) ; G$ et $G_{1}$ s'identifient donc aux groupes algébriques correspondants, que nous noterons pour abréger $G L_{m}$ et $S L_{m}$; pour abréger aussi, on écrira $F$ au lieu de $\left(S L_{m}\right)_{A} /\left(S L_{m}\right)_{k}$. Alors $I_{1}(\Phi)$ s'écrit :

$$
I_{1}(\Phi)=\int_{F} \sum_{\eta, \zeta} \Phi\left(u \eta,{ }^{t} u^{\prime-1} \zeta\right) \cdot d \mu(u),
$$

où la sommation est étendue à $\eta \in Y_{k}, \zeta \in Z_{k}$, et où $\mu$ est la mesure de Haar sur $\left(S L_{m}\right)_{A}$, normée par $\mu(F)=1$. Comme on sait, 1 est un système de facteurs de convergence pour $S L_{m}$; on posera $\tau_{m}=\tau_{1}\left(S L_{m}\right)$; c'est le nombre de Tamagawa de $S L_{m}$; si $d u$ est une jauge invariante sur $S L_{m}$, on a $d \mu(u)=\tau_{m}^{-1}|d u|_{A}$.

D'après la proposition $3 \mathrm{du}$ Chapitre II, $\mathrm{n}^{\circ} 22, G_{k}$ a deux orbites dans $Y_{k}$, à savoir $\{0\}$ et l'orbite $U_{k}^{\prime}=Y_{k}-\{0\}$ des points de rang maximal dans $Y_{k}$. Si on étend $\mathcal{A}_{k}$ et $Y_{k}$ au domaine universel, et qu'on note $U^{\prime}$ l'ensemble des points de rang maximal dans $Y$, $U^{\prime}$ est une orbite de $G$, et $U_{k}^{\prime}$ est l'ensemble des points de $U^{\prime}$ qui sont rationnels sur $k$. De même, pour tout corps $K \supset k$, l'ensemble $U_{K}^{\prime}$ des points de $U^{\prime}$ qui sont rationnels sur $K$ coïncide avec l'ensemble des points de rang maximal de $Y_{K}$ et est donc une orbite de $G_{K}$. De plus, on vérifie immédiatement que le groupe $\left(G_{1}\right)_{K}$ opère transitivement sur $U_{K}^{\prime}$. De même, si $U^{\prime \prime}$ est l'ensemble des points de rang maximal de $Z, U_{K}^{\prime \prime}$ est une orbite de $G_{K}$, et aussi de $\left(G_{1}\right)_{K}$, quel que soit $K \supset k$, et on a $U_{k}^{\prime \prime}=Z_{k}-\{0\}$.

D'après le $\mathrm{n}^{\circ} 21$ du Chapitre II, on peut ici identifier $I(X)_{k}$ avec f. D'après la proposition 3 du Chapitre II, $\mathrm{n}^{\circ} 22$, et son corollaire, les orbites de $G_{k}$ dans $X_{k}$ sont d'une part les 
$U(i)_{k}$ pour $i \in I(X)_{k}$, et d'autre part les trois orbites $U_{k}^{\prime} \times\{0\},\{0\} \times U_{k}^{\prime \prime}$ et $\{0\}$, On vérifie immédiatement que $\left(G_{1}\right)_{k}$ opère transitivement sur ces orbites et qu'elles satisfont à la condition $(W)$ du Chapitre $I, n^{\circ} 8$. La détermination des groupes de stabilité des points de ces orbites dans $G_{1}$ se fait sans difficulté, soit au moyen du $n^{\circ} 23$ du Chapitre II, soit directement; ces groupes sont respectivement isomorphes à $S L_{m-1}$ (pour les points des orbites $U(i)_{k}$ avec $i \neq 0$ ), à un produit semidirect de $S L_{m-2}$ et d'un groupe unipotent (pour les points de $U(0)_{k}$ ), et à un produit semidirect de $S L_{m-1}$ et d'un groupe unipotent (pour les points de $U_{k}^{\prime} \times\{0\}$ et de $\left.\{0\} \times U_{k}^{\prime \prime}\right)$. Les résultats du Chapitre $\mathrm{I}, \mathrm{n}^{\text {os }} \mathbf{7 - 8}$, donnent alors, en raisonnant comme au $n^{\circ} 53$ :

$$
I_{1}(\Phi)=\Phi(0,0)+c^{\prime} \int_{U_{A}^{\prime}} \Phi(y, 0)|d y|_{A}+c^{\prime \prime} \int_{U_{A}^{\prime \prime}} \Phi(0, z)|d z|_{A}+\sum_{i \in \mathfrak{t}} c_{i} \int_{U(i) A} \Phi \cdot\left|\theta_{i}\right|_{A}
$$

avec $c_{i}=\tau_{m-1} / \tau_{m}$ pour $i \neq 0, c_{0}=\tau_{m-2} / \tau_{m}$, et $c^{\prime}=c^{\prime \prime}=\tau_{m-1} / \tau_{m}$.

Les théorèmes d'unicité relatifs au cas où nous nous plaçons ici, théorèmes auxquels il a été fait allusion au début du Chapitre $V$, permettraient de conclure de là qu'on a $c_{i}=1$ quel que soit $i$, donc que $\tau_{m}$ est indépendant de $m$ pour $m \geqslant 1$. Comme en fait il est bien connu qu'on a même $\tau_{m}=1$ quel que soit $m$ (cf. [13], Chap. III), cela n'apprendrait rien de nouveau. De quelque manière qu'on procède, on voit que les coefficients $c_{i}, c^{\prime}, c^{\prime \prime}$ de la formule ci-dessus ont tous la valeur 1 . Comme on sait aussi que $U_{A}^{\prime}, U_{A}^{\prime \prime}$ sont les complémentaires d'ensembles négligeables dans $Y_{A}$ et $Z_{A}$, pour les mesures $|d y|_{A}$ et $|d z|_{A}$ respectivement (cf. [13], Lemma 3.4.1), on obtient donc en définitive $\left(^{1}\right)$ :

$$
I_{1}(\Phi)=E(\Phi)+\int_{Y_{A}} \Phi(y, 0)|d y|_{A}+\int_{z_{A}} \Phi(0, z)\left|d z_{A}\right| .
$$

\section{Bibliographie}

[1]. Albert, A. A., Structure of algebras. Amer. Math. Soc. Colloquium Series, vol. XXIV, Providence, Amer. Math. Soc. 1961.

[2]. Borel, A., Some finiteness properties of adele groups over number-fields. Publ. Math. I.H.E.S. $\mathrm{n}^{\circ} 16,1963$.

[3]. Borel, A. \& Harish-Chandra, Arithmetic subgroups of algebraic groups. Ann. of Math., 75 (1962), 485-535.

[4]. BourbakI, N., Intégration, Chapitre VII: Mesure de Haar. Paris, Hermann, 1963.

[5]. Dieudonnte, J., La Géométrie des Groupes Classiques. Ergeb. d. Math. (N.F.) Bd. 5, 2éd., J. Springer 1963.

(1) Je saisis cette occasion pour indiquer, à la prière de Siegel, que la formule de [12], p. 378, ligne 4 du bas, doit être corrigée comme suit: au second membre, remplacer l'exposant - $m$ par $-m / 2$, ajouter un terme $+2 y^{-m / 2}$, et remplacer la condition $m>1$ par $m>2$. Après cette rectification, qui m'a été communiquée par Siegel il y a quelque temps déjà, sa formule devient un cas particulier de celle du texte. 
[6]. Godement, R., Domaines fondamentaux des groupes arithmétiques. Séminaire Bourbaki, $15^{e}$ année (1962-63), $\mathrm{n}^{\circ} 257$.

[7]. Convergence des séries d'Eisenstein. A paraître.

[8]. KNeser, M., Starke Approximation in algebraischen Gruppen. A paraître, Crelles $J$.

[9]. - Galois-Kohomologie halbeinfacher algebraischen Gruppen über p-adischen Körpern I. A paraître, Math. Zeitschr.

[10]. Langweil, S. A., Number of points of varieties in finite fields. Amer. J. of Math., 76 (1954), 819-827.

[11]. Rosenticht, M., Some rationality questions on algebraic groups. Ann. di Mat. Pura e Appl. (IV), 43 (1957), 25-50.

[12]. Siegel, C. L., Indefinite quadratische Formen und Funktionentheorie. Math. Ann., 124 (1952), 17-54 et 364-387.

[13]. WeIL, A., Adèles and algebraic groups. Princeton, Inst. for Adv. Study 1961.

[14]. — Sur certains groupes d'opérateurs unitaires. Acta Math., 111 (1964), 143-211.

$R e_{s}^{\wedge} u$ le 8 juin 1964 